\title{
Synthetic, Structural, and Mechanistic Aspects of an Amine Activation Process Mediated at a Zwitterionic Pd(II) Center
}

\author{
Supporting Information
}

Connie C. Lu and Jonas C. Peters*

Division of Chemistry and Chemical Engineering, Arnold and Mabel Beckman Laboratories of Chemical Synthesis, California Institute of

Technology, Pasadena, CA 91125

Table of Contents

(1) Figure 1. Fully labeled drawing of 1'

(2) Table 1. Crystal data and structure refinement for 1'

(3) Table 2. Atomic coordinates and equivalent isotropic displacement parameters for 1'

(4) Table 3. Bond lengths and angles for 1'

(5) Table 4. Anisotropic parameters for 1'

(6) Table 5. Hydrogen coordinates and isotropic displacement parameters for $\mathbf{1}^{\prime}$

(7) Figure 2. Fully labeled drawing of 5

(8) Table 6. Crystal data and structure refinement for 5

(9) Table 7. Atomic coordinates and equivalent isotropic displacement parameters for 5

(10) Table 8. Bond lengths and angles for $\mathbf{5}$

(11) Table 9. Anisotropic parameters for $\mathbf{5}$

(12) Table 10. Hydrogen coordinates and isotropic displacement parameters for 5

(13) Figure 3. Fully labeled drawing of 8

(14) Table 11. Crystal data and structure refinement for $\mathbf{8}$

(15) Table 12. Atomic coordinates and equivalent isotropic displacement parameters for $\mathbf{8}$

(16) Table 13. Bond lengths and angles for 8

(17) Table 14. Anisotropic parameters for $\mathbf{8}$

(18) Table 15. Hydrogen coordinates and isotropic displacement parameters for 8

(19) Figure 4. Fully labeled drawing of 9

(20) Table 16. Crystal data and structure refinement for 9

(21) Table 17. Atomic coordinates and equivalent isotropic displacement parameters for 9

(22) Table 18. Bond lengths and angles for 9

(23) Table 19. Anisotropic parameters for 9

(24) Table 20. Hydrogen coordinates and isotropic displacement parameters for 9 
Figure 1. Labeled drawing of 1' (CCDC 171286), with 50\% ellipsoids. Hydrogen atoms and solvent molecule (THF) have been omitted for clarity.

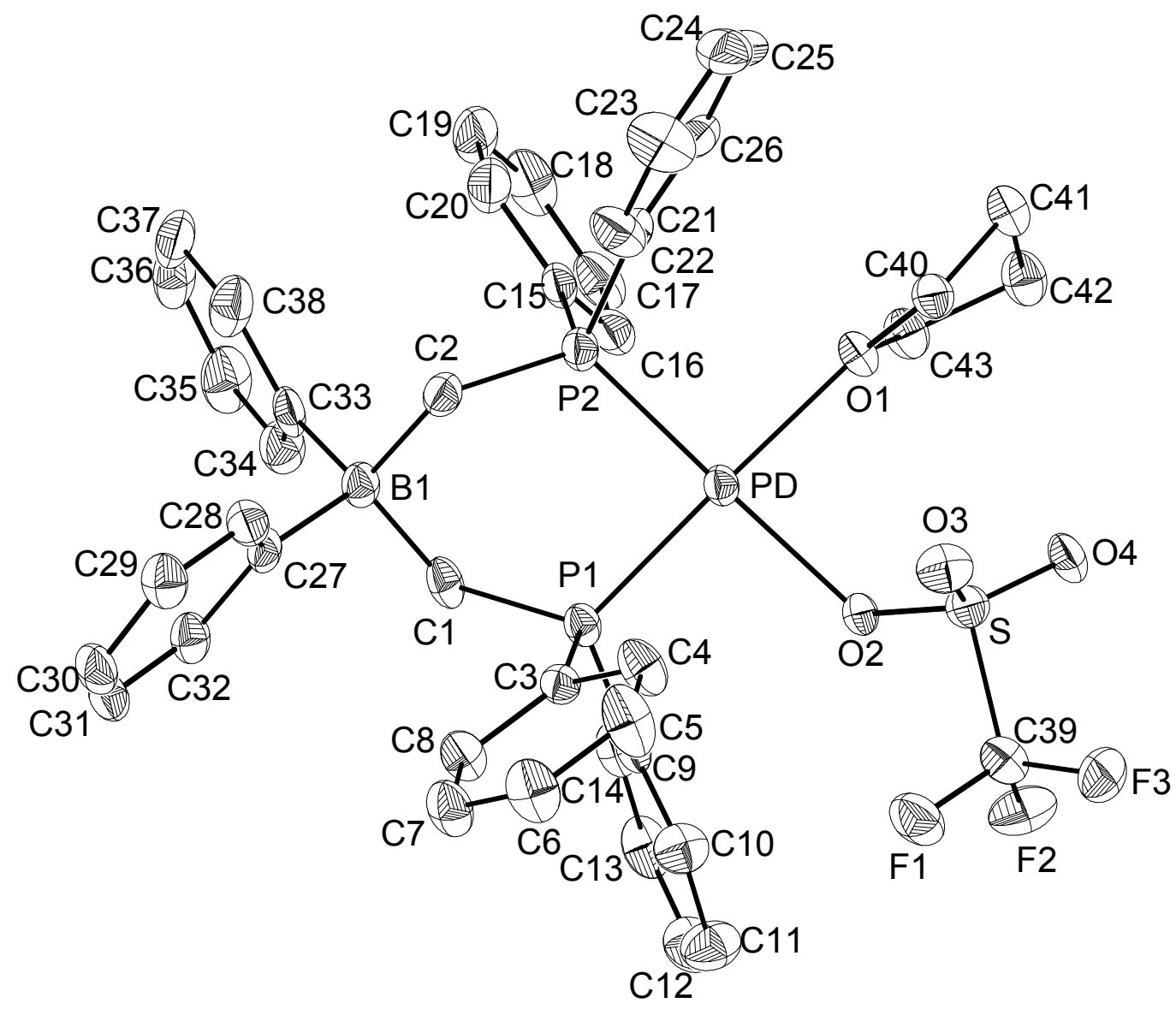


Table 1. Crystal data and structure refinement for 1' (CCDC 171286).

Empirical formula

Formula weight

Crystallization Solvent

Crystal Habit

Crystal size

Crystal color

Preliminary Photos

Type of diffractometer

Wavelength

Data Collection Temperature

$\theta$ range for 22373 reflections used in lattice determination

Unit cell dimensions

Volume

Z

Crystal system

Space group

Density (calculated)

$\mathrm{F}(000)$

Data collection program

$\theta$ range for data collection

Completeness to $\theta=28.50^{\circ}$

Index ranges

Data collection scan type

Data reduction program

Reflections collected

Independent reflections

Absorption coefficient

Absorption correction

Max. and min. transmission
$\mathrm{C}_{43} \mathrm{H}_{42} \mathrm{BF}_{3} \mathrm{O}_{4} \mathrm{P}_{2} \mathrm{SPd} \cdot \mathrm{C}_{4} \mathrm{H}_{8} \mathrm{O}$

963.08

THF/Petroleum ether

Fragment

$0.31 \times 0.22 \times 0.19 \mathrm{~mm}^{3}$

Colorless

\section{Data Collection}

Rotation

CCD area detector

$0.71073 \AA \mathrm{MoK} \alpha$

98(2) K

2.28 to $27.84^{\circ}$

$a=13.2666(7) \AA$

$\mathrm{b}=17.4832(10) \AA$

$\mathrm{c}=19.2115(10) \AA$

$\beta=95.8550(10)^{\circ}$

4432.7(4) $\AA^{3}$

4

Monoclinic

$\mathrm{P} 2{ }_{1} / \mathrm{n}$

$1.443 \mathrm{Mg} / \mathrm{m}^{3}$

1984

Bruker SMART

1.58 to $28.50^{\circ}$

$95.0 \%$

$-17 \leq \mathrm{h} \leq 17,-23 \leq \mathrm{k} \leq 23,-25 \leq 1 \leq 25$

$\omega$ scans at $7 \phi$ settings

Bruker SAINT v6.2

90844

$10664\left[\mathrm{R}_{\text {int }}=0.0815\right]$

$0.596 \mathrm{~mm}^{-1}$

None

0.8941 and 0.8344 


\section{Table 1 (cont.)}

\section{Structure solution and Refinement}

Structure solution program

Primary solution method

Secondary solution method

Hydrogen placement

Structure refinement program

Refinement method

Data / restraints / parameters

Treatment of hydrogen atoms

Goodness-of-fit on $\mathrm{F}^{2}$

Final $\mathrm{R}$ indices $[\mathrm{I}>2 \sigma(\mathrm{I}), 7182$ reflections $]$

$\mathrm{R}$ indices (all data)

Type of weighting scheme used

Weighting scheme used

Max shift/error

Average shift/error

Largest diff. peak and hole
SHELXS-97 (Sheldrick, 1990)

Patterson method

Difference Fourier map

Difference Fourier map

SHELXL-97 (Sheldrick, 1997)

Full matrix least-squares on $\mathrm{F}^{2}$

10664 / $10 / 709$

Unrestrained except on solvent

1.743

$\mathrm{R} 1=0.0478, w \mathrm{R} 2=0.0701$

$\mathrm{R} 1=0.0805, w \mathrm{R} 2=0.0728$

Sigma

$w=1 / \sigma^{2}\left(\mathrm{Fo}^{2}\right)$

0.061

0.002

1.121 and -0.923 e. $\AA^{-3}$

\section{Special Refinement Details}

The crystals contain THF as a solvent of crystallization. The geometry of the solvent THF was restrained to be similar to the geometry of the bond THF and the hydrogen atoms of the solvent THF were restrained to ideal geometry.

Refinement of $\mathrm{F}^{2}$ against ALL reflections. The weighted R-factor $(w \mathrm{R})$ and goodness of fit $(\mathrm{S})$ are based on $\mathrm{F}^{2}$, conventional R-factors $(\mathrm{R})$ are based on $\mathrm{F}$, with $\mathrm{F}$ set to zero for negative $\mathrm{F}^{2}$. The threshold expression of $\mathrm{F}^{2}>$ $2 \sigma\left(\mathrm{F}^{2}\right)$ is used only for calculating R-factors $(\mathrm{gt})$ etc. and is not relevant to the choice of reflections for refinement. $\mathrm{R}$-factors based on $\mathrm{F}^{2}$ are statistically about twice as large as those based on $\mathrm{F}$, and R-factors based on ALL data will be even larger.

All esds (except the esd in the dihedral angle between two l.s. planes) are estimated using the full covariance matrix. The cell esds are taken into account individually in the estimation of esds in distances, angles and torsion angles; correlations between esds in cell parameters are only used when they are defined by crystal symmetry. An approximate (isotropic) treatment of cell esds is used for estimating esds involving l.s. planes. 
Table 2. Atomic coordinates $\left(\times 10^{4}\right)$ and equivalent isotropic displacement parameters $\left(\AA^{2} \times 1^{3}\right)$ for $1^{\prime}(C C D C 171286)$. $U(e q)$ the trace of the orthogonalized $U^{i j}$ tensor.

\begin{tabular}{|c|c|c|c|c|}
\hline & $\mathrm{x}$ & $\mathrm{y}$ & $\mathrm{z}$ & $\mathrm{U}_{\text {eq }}$ \\
\hline $\mathrm{Pd}$ & $5290(1)$ & $1940(1)$ & $9470(1)$ & $21(1)$ \\
\hline $\mathrm{S}$ & $3144(1)$ & $1158(1)$ & $9594(1)$ & $27(1)$ \\
\hline $\mathrm{P}(1)$ & $5415(1)$ & $2214(1)$ & $8345(1)$ & $22(1)$ \\
\hline $\mathrm{P}(2)$ & $6602(1)$ & $2713(1)$ & $9768(1)$ & $24(1)$ \\
\hline $\mathrm{B}(1)$ & $7209(3)$ & $3246(2)$ & $8424(2)$ & $25(1)$ \\
\hline $\mathrm{F}(1)$ & 2095(1) & $1374(1)$ & $8395(1)$ & $42(1)$ \\
\hline $\mathrm{F}(2)$ & 2393(1) & 190(1) & $8651(1)$ & $45(1)$ \\
\hline $\mathrm{F}(3)$ & $1279(1)$ & $806(1)$ & $9165(1)$ & $39(1)$ \\
\hline $\mathrm{O}(2)$ & $4043(1)$ & $1170(1)$ & $9202(1)$ & $24(1)$ \\
\hline $\mathrm{O}(3)$ & $2827(2)$ & $1905(1)$ & $9796(1)$ & $33(1)$ \\
\hline $\mathrm{O}(4)$ & $3149(2)$ & 553(1) & $10098(1)$ & $30(1)$ \\
\hline $\mathrm{C}(1)$ & $6658(2)$ & $2452(2)$ & $8126(2)$ & $26(1)$ \\
\hline $\mathrm{C}(2)$ & $6792(3)$ & $3477(2)$ & $9175(2)$ & $27(1)$ \\
\hline$C(3)$ & $4517(2)$ & $2952(2)$ & $8062(2)$ & $22(1)$ \\
\hline$C(4)$ & $3709(3)$ & $3122(2)$ & $8441(2)$ & $34(1)$ \\
\hline$C(5)$ & 2988(3) & $3645(2)$ & $8190(2)$ & $44(1)$ \\
\hline$C(6)$ & $3058(3)$ & $4009(2)$ & $7566(2)$ & $39(1)$ \\
\hline$C(7)$ & $3850(3)$ & $3852(2)$ & $7183(2)$ & $36(1)$ \\
\hline $\mathrm{C}(8)$ & $4578(2)$ & $3324(2)$ & $7428(2)$ & $30(1)$ \\
\hline C(9) & $5030(2)$ & $1396(2)$ & $7802(2)$ & $24(1)$ \\
\hline$C(10)$ & $4066(3)$ & $1334(2)$ & $7459(2)$ & $34(1)$ \\
\hline $\mathrm{C}(11)$ & $3818(3)$ & $735(2)$ & $7005(2)$ & $41(1)$ \\
\hline C(12) & $4535(3)$ & $196(2)$ & $6883(2)$ & $41(1)$ \\
\hline$C(13)$ & $5487(3)$ & $239(2)$ & $7221(2)$ & $36(1)$ \\
\hline$C(14)$ & $5743(3)$ & $832(2)$ & $7677(2)$ & $31(1)$ \\
\hline$C(15)$ & $7741(2)$ & $2149(2)$ & $9949(2)$ & $27(1)$ \\
\hline$C(16)$ & $7754(3)$ & $1373(2)$ & $9807(2)$ & $30(1)$ \\
\hline$C(17)$ & $8621(3)$ & $942(2)$ & $9965(2)$ & $37(1)$ \\
\hline $\mathrm{C}(18)$ & $9485(3)$ & $1285(3)$ & $10264(2)$ & $46(1)$ \\
\hline C(19) & $9492(3)$ & $2057(3)$ & $10406(2)$ & $47(1)$ \\
\hline $\mathrm{C}(20)$ & $8625(3)$ & $2493(2)$ & $10253(2)$ & $37(1)$ \\
\hline $\mathrm{C}(21)$ & $6392(2)$ & $3143(2)$ & $10597(2)$ & $26(1)$ \\
\hline$C(22)$ & $5860(3)$ & $3824(2)$ & $10607(2)$ & $40(1)$ \\
\hline$C(23)$ & $5673(3)$ & $4156(3)$ & $11237(2)$ & $48(1)$ \\
\hline$C(24)$ & $6009(3)$ & $3809(2)$ & $11856(2)$ & $40(1)$ \\
\hline$C(25)$ & $6534(3)$ & $3132(2)$ & $11858(2)$ & $33(1)$ \\
\hline$C(26)$ & $6734(2)$ & $2801(2)$ & $11232(2)$ & $29(1)$ \\
\hline$C(27)$ & $6973(2)$ & $3943(2)$ & $7860(2)$ & $24(1)$ \\
\hline$C(28)$ & $6580(2)$ & $4649(2)$ & $8022(2)$ & $30(1)$ \\
\hline C(29) & $6389(2)$ & $5230(2)$ & $7525(2)$ & $33(1)$ \\
\hline C(30) & $6589(3)$ & $5110(2)$ & $6847(2)$ & $34(1)$ \\
\hline$C(31)$ & $7001(3)$ & $4420(2)$ & $6669(2)$ & $33(1)$ \\
\hline $\mathrm{C}(32)$ & $7190(2)$ & $3860(2)$ & $7167(2)$ & $28(1)$ \\
\hline C(33) & $8437(2)$ & $3100(2)$ & $8533(2)$ & $27(1)$ \\
\hline $\mathrm{C}(34)$ & $8915(3)$ & $2476(2)$ & $8263(2)$ & $36(1)$ \\
\hline$C(35)$ & 9951(3) & $2346(3)$ & $8394(2)$ & $44(1)$ \\
\hline$C(36)$ & $10552(3)$ & $2836(2)$ & $8798(2)$ & $46(1)$ \\
\hline$C(37)$ & $10124(3)$ & $3473(3)$ & $9055(2)$ & $44(1)$ \\
\hline
\end{tabular}




\begin{tabular}{lrrrr}
$\mathrm{C}(38)$ & $9088(3)$ & $3606(2)$ & $8924(2)$ & $39(1)$ \\
$\mathrm{C}(39)$ & $2180(2)$ & $866(2)$ & $8919(2)$ & $30(1)$ \\
$\mathrm{O}(1)$ & $5217(1)$ & $1584(1)$ & $10539(1)$ & $23(1)$ \\
$\mathrm{C}(40)$ & $4637(3)$ & $1847(2)$ & $11105(2)$ & $26(1)$ \\
$\mathrm{C}(41)$ & $4986(3)$ & $1338(2)$ & $11721(2)$ & $30(1)$ \\
$\mathrm{C}(42)$ & $5184(3)$ & $598(2)$ & $11352(2)$ & $32(1)$ \\
$\mathrm{C}(43)$ & $838(2)$ & $10713(2)$ & $29(1)$ \\
$\mathrm{O}(5)$ & & & \\
$\mathrm{C}(51)$ & $5659(3)$ & & $5178(3)$ & $173(2)$ \\
$\mathrm{C}(52)$ & $6991(4)$ & $428(2)$ & $5763(3)$ & $200(5)$ \\
$\mathrm{C}(53)$ & $6547(5)$ & $893(6)$ & $5848(3)$ & $163(4)$ \\
$\mathrm{C}(54)$ & $7146(5)$ & $1600(4)$ & $5518(4)$ & $120(2)$ \\
& $8060(4)$ & $1384(3)$ & $5101(3)$ & $124(3)$ \\
\hline
\end{tabular}


Table 3. Bond lengths $[\AA ̊ \AA]$ and angles $\left[^{\circ}\right]$ for $1^{\prime}$ (CCDC 171286).

\begin{tabular}{|c|c|c|c|}
\hline $\mathrm{Pd}-\mathrm{O}(2)$ & $2.155(2)$ & $\mathrm{C}(17)-\mathrm{C}(18)$ & $1.368(5)$ \\
\hline $\mathrm{Pd}-\mathrm{O}(1)$ & $2.157(2)$ & $\mathrm{C}(17)-\mathrm{H}(17)$ & $0.92(3)$ \\
\hline Pd-P(2) & $2.2309(9)$ & $C(18)-C(19)$ & $1.377(5)$ \\
\hline Pd-P(1) & $2.2378(8)$ & $\mathrm{C}(18)-\mathrm{H}(18)$ & $0.96(3)$ \\
\hline $\mathrm{S}-\mathrm{O}(4)$ & $1.434(2)$ & $C(19)-C(20)$ & $1.387(5)$ \\
\hline $\mathrm{S}-\mathrm{O}(3)$ & $1.438(2)$ & $\mathrm{C}(19)-\mathrm{H}(19)$ & $0.88(3)$ \\
\hline $\mathrm{S}-\mathrm{O}(2)$ & $1.474(2)$ & $\mathrm{C}(20)-\mathrm{H}(20)$ & $0.90(3)$ \\
\hline S-C(39) & $1.800(3)$ & $C(21)-C(22)$ & $1.385(4)$ \\
\hline $\mathrm{P}(1)-\mathrm{C}(1)$ & $1.792(3)$ & $C(21)-C(26)$ & $1.392(4)$ \\
\hline $\mathrm{P}(1)-\mathrm{C}(3)$ & $1.802(3)$ & $C(22)-C(23)$ & $1.387(5)$ \\
\hline $\mathrm{P}(1)-\mathrm{C}(9)$ & $1.812(3)$ & $\mathrm{C}(22)-\mathrm{H}(22)$ & $0.95(3)$ \\
\hline $\mathrm{P}(2)-\mathrm{C}(2)$ & $1.790(3)$ & $C(23)-C(24)$ & $1.368(5)$ \\
\hline $\mathrm{P}(2)-\mathrm{C}(15)$ & $1.809(3)$ & $\mathrm{C}(23)-\mathrm{H}(23)$ & $0.85(3)$ \\
\hline $\mathrm{P}(2)-\mathrm{C}(21)$ & $1.809(3)$ & $C(24)-C(25)$ & $1.374(5)$ \\
\hline $\mathrm{B}(1)-\mathrm{C}(27)$ & $1.640(4)$ & $\mathrm{C}(24)-\mathrm{H}(24)$ & $0.96(3)$ \\
\hline $\mathrm{B}(1)-\mathrm{C}(33)$ & $1.641(4)$ & $C(25)-C(26)$ & $1.384(4)$ \\
\hline B(1)-C(1) & $1.645(5)$ & $\mathrm{C}(25)-\mathrm{H}(25)$ & $0.97(3)$ \\
\hline $\mathrm{B}(1)-\mathrm{C}(2)$ & $1.646(5)$ & $\mathrm{C}(26)-\mathrm{H}(26)$ & $0.92(3)$ \\
\hline $\mathrm{F}(1)-\mathrm{C}(39)$ & $1.338(3)$ & $C(27)-C(28)$ & $1.387(4)$ \\
\hline $\mathrm{F}(2)-\mathrm{C}(39)$ & $1.330(3)$ & $C(27)-C(32)$ & $1.398(4)$ \\
\hline $\mathrm{F}(3)-\mathrm{C}(39)$ & $1.334(3)$ & $C(28)-C(29)$ & $1.399(4)$ \\
\hline C(1)-H(1A) & $0.93(3)$ & $\mathrm{C}(28)-\mathrm{H}(28)$ & $1.00(3)$ \\
\hline $\mathrm{C}(1)-\mathrm{H}(1 \mathrm{~B})$ & $0.94(3)$ & $C(29)-C(30)$ & $1.371(4)$ \\
\hline $\mathrm{C}(2)-\mathrm{H}(2 \mathrm{~A})$ & $0.95(3)$ & $\mathrm{C}(29)-\mathrm{H}(29)$ & $0.96(3)$ \\
\hline $\mathrm{C}(2)-\mathrm{H}(2 \mathrm{~B})$ & $0.90(3)$ & $C(30)-C(31)$ & $1.381(5)$ \\
\hline$C(3)-C(4)$ & $1.388(4)$ & $\mathrm{C}(30)-\mathrm{H}(30)$ & $0.91(3)$ \\
\hline$C(3)-C(8)$ & $1.391(4)$ & $C(31)-C(32)$ & $1.374(4)$ \\
\hline$C(4)-C(5)$ & $1.375(4)$ & $\mathrm{C}(31)-\mathrm{H}(31)$ & $0.85(3)$ \\
\hline $\mathrm{C}(4)-\mathrm{H}(4)$ & $0.95(3)$ & $\mathrm{C}(32)-\mathrm{H}(32)$ & $0.90(2)$ \\
\hline$C(5)-C(6)$ & $1.368(5)$ & $C(33)-C(34)$ & $1.389(5)$ \\
\hline $\mathrm{C}(5)-\mathrm{H}(5)$ & $0.85(3)$ & $C(33)-C(38)$ & $1.399(5)$ \\
\hline$C(6)-C(7)$ & $1.370(5)$ & $C(34)-C(35)$ & $1.391(5)$ \\
\hline $\mathrm{C}(6)-\mathrm{H}(6)$ & $0.84(3)$ & $\mathrm{C}(34)-\mathrm{H}(34)$ & $0.91(3)$ \\
\hline $\mathrm{C}(7)-\mathrm{C}(8)$ & $1.384(4)$ & $C(35)-C(36)$ & $1.357(5)$ \\
\hline $\mathrm{C}(7)-\mathrm{H}(7)$ & $1.02(3)$ & $\mathrm{C}(35)-\mathrm{H}(35)$ & $0.91(3)$ \\
\hline $\mathrm{C}(8)-\mathrm{H}(8)$ & $0.95(3)$ & $C(36)-C(37)$ & $1.366(5)$ \\
\hline$C(9)-C(10)$ & $1.383(4)$ & $\mathrm{C}(36)-\mathrm{H}(36)$ & $0.97(3)$ \\
\hline C(9)-C(14) & $1.404(4)$ & $\mathrm{C}(37)-\mathrm{C}(38)$ & $1.391(5)$ \\
\hline $\mathrm{C}(10)-\mathrm{C}(11)$ & $1.381(5)$ & $\mathrm{C}(37)-\mathrm{H}(37)$ & $0.97(3)$ \\
\hline $\mathrm{C}(10)-\mathrm{H}(10)$ & $0.87(3)$ & $\mathrm{C}(38)-\mathrm{H}(38)$ & $0.91(2)$ \\
\hline C(11)-C(12) & $1.376(5)$ & $\mathrm{O}(1)-\mathrm{C}(43)$ & $1.454(3)$ \\
\hline $\mathrm{C}(11)-\mathrm{H}(11)$ & $0.89(3)$ & $\mathrm{O}(1)-\mathrm{C}(40)$ & $1.469(3)$ \\
\hline C(12)-C(13) & $1.361(5)$ & $\mathrm{C}(40)-\mathrm{C}(41)$ & $1.514(4)$ \\
\hline $\mathrm{C}(12)-\mathrm{H}(12)$ & $0.88(3)$ & $\mathrm{C}(40)-\mathrm{H}(40 \mathrm{~A})$ & $0.94(3)$ \\
\hline$C(13)-C(14)$ & $1.378(5)$ & $\mathrm{C}(40)-\mathrm{H}(40 \mathrm{~B})$ & $1.02(3)$ \\
\hline $\mathrm{C}(13)-\mathrm{H}(13)$ & $0.90(3)$ & $\mathrm{C}(41)-\mathrm{C}(42)$ & $1.511(4)$ \\
\hline $\mathrm{C}(14)-\mathrm{H}(14)$ & $0.96(3)$ & $\mathrm{C}(41)-\mathrm{H}(41 \mathrm{~A})$ & $1.03(3)$ \\
\hline$C(15)-C(16)$ & $1.385(4)$ & $\mathrm{C}(41)-\mathrm{H}(41 \mathrm{~B})$ & $1.00(3)$ \\
\hline$C(15)-C(20)$ & $1.391(4)$ & C(42)-C(43) & $1.496(4)$ \\
\hline$C(16)-C(17)$ & $1.383(4)$ & $\mathrm{C}(42)-\mathrm{H}(42 \mathrm{~A})$ & $0.91(3)$ \\
\hline $\mathrm{C}(16)-\mathrm{H}(16)$ & $0.97(2)$ & $\mathrm{C}(42)-\mathrm{H}(42 \mathrm{~B})$ & $0.98(3)$ \\
\hline
\end{tabular}




\begin{tabular}{|c|c|c|c|}
\hline $\mathrm{C}(43)-\mathrm{H}(43 \mathrm{~A})$ & $0.94(3)$ & $\mathrm{B}(1)-\mathrm{C}(2)-\mathrm{H}(2 \mathrm{~A})$ & $109.5(16)$ \\
\hline $\mathrm{C}(43)-\mathrm{H}(43 \mathrm{~B})$ & $1.06(3)$ & $\mathrm{P}(2)-\mathrm{C}(2)-\mathrm{H}(2 \mathrm{~A})$ & $103.5(16)$ \\
\hline $\mathrm{O}(5)-\mathrm{C}(54)$ & $1.380(5)$ & $\mathrm{B}(1)-\mathrm{C}(2)-\mathrm{H}(2 \mathrm{~B})$ & $113.3(17)$ \\
\hline $\mathrm{O}(5)-\mathrm{C}(51)$ & $1.550(7)$ & $\mathrm{P}(2)-\mathrm{C}(2)-\mathrm{H}(2 \mathrm{~B})$ & $105.6(17)$ \\
\hline $\mathrm{C}(51)-\mathrm{C}(52)$ & $1.470(9)$ & $\mathrm{H}(2 \mathrm{~A})-\mathrm{C}(2)-\mathrm{H}(2 \mathrm{~B})$ & $107(2)$ \\
\hline $\mathrm{C}(51)-\mathrm{H}(51 \mathrm{~A})$ & 0.9900 & $\mathrm{C}(4)-\mathrm{C}(3)-\mathrm{C}(8)$ & $118.5(3)$ \\
\hline $\mathrm{C}(51)-\mathrm{H}(51 \mathrm{~B})$ & 0.9900 & $\mathrm{C}(4)-\mathrm{C}(3)-\mathrm{P}(1)$ & $121.1(2)$ \\
\hline $\mathrm{C}(52)-\mathrm{C}(53)$ & $1.473(7)$ & $\mathrm{C}(8)-\mathrm{C}(3)-\mathrm{P}(1)$ & $120.2(2)$ \\
\hline $\mathrm{C}(52)-\mathrm{H}(52 \mathrm{~A})$ & 0.9900 & $C(5)-C(4)-C(3)$ & $120.3(3)$ \\
\hline $\mathrm{C}(52)-\mathrm{H}(52 \mathrm{~B})$ & 0.9900 & $\mathrm{C}(5)-\mathrm{C}(4)-\mathrm{H}(4)$ & $121.5(19)$ \\
\hline $\mathrm{C}(53)-\mathrm{C}(54)$ & $1.430(6)$ & $\mathrm{C}(3)-\mathrm{C}(4)-\mathrm{H}(4)$ & 118.2(19) \\
\hline $\mathrm{C}(53)-\mathrm{H}(53 \mathrm{~A})$ & 0.9900 & $C(6)-C(5)-C(4)$ & $120.6(4)$ \\
\hline $\mathrm{C}(53)-\mathrm{H}(53 \mathrm{~B})$ & 0.9900 & $\mathrm{C}(6)-\mathrm{C}(5)-\mathrm{H}(5)$ & $116(2)$ \\
\hline $\mathrm{C}(54)-\mathrm{H}(54 \mathrm{~A})$ & 0.9900 & $\mathrm{C}(4)-\mathrm{C}(5)-\mathrm{H}(5)$ & $123(2)$ \\
\hline \multirow[t]{2}{*}{$\mathrm{C}(54)-\mathrm{H}(54 \mathrm{~B})$} & 0.9900 & $C(5)-C(6)-C(7)$ & $120.2(4)$ \\
\hline & & $\mathrm{C}(5)-\mathrm{C}(6)-\mathrm{H}(6)$ & $117(2)$ \\
\hline $\mathrm{O}(2)-\mathrm{Pd}-\mathrm{O}(1)$ & $86.52(7)$ & $\mathrm{C}(7)-\mathrm{C}(6)-\mathrm{H}(6)$ & $122(2)$ \\
\hline O(2)-Pd-P(2) & $178.42(6)$ & $C(6)-C(7)-C(8)$ & 119.9(3) \\
\hline $\mathrm{O}(1)-\mathrm{Pd}-\mathrm{P}(2)$ & $92.33(6)$ & $\mathrm{C}(6)-\mathrm{C}(7)-\mathrm{H}(7)$ & $120.6(17)$ \\
\hline $\mathrm{O}(2)-\mathrm{Pd}-\mathrm{P}(1)$ & $91.90(5)$ & $\mathrm{C}(8)-\mathrm{C}(7)-\mathrm{H}(7)$ & $119.5(17)$ \\
\hline O(1)-Pd-P(1) & $175.29(6)$ & $C(7)-C(8)-C(3)$ & $120.6(3)$ \\
\hline $\mathrm{P}(2)-\mathrm{Pd}-\mathrm{P}(1)$ & $89.16(3)$ & $\mathrm{C}(7)-\mathrm{C}(8)-\mathrm{H}(8)$ & $119.5(17)$ \\
\hline $\mathrm{O}(4)-\mathrm{S}-\mathrm{O}(3)$ & $118.04(13)$ & $\mathrm{C}(3)-\mathrm{C}(8)-\mathrm{H}(8)$ & $120.0(17)$ \\
\hline $\mathrm{O}(4)-\mathrm{S}-\mathrm{O}(2)$ & $114.07(12)$ & $C(10)-C(9)-C(14)$ & $118.0(3)$ \\
\hline $\mathrm{O}(3)-\mathrm{S}-\mathrm{O}(2)$ & $113.46(12)$ & $\mathrm{C}(10)-\mathrm{C}(9)-\mathrm{P}(1)$ & $121.9(2)$ \\
\hline $\mathrm{O}(4)-\mathrm{S}-\mathrm{C}(39)$ & $103.42(14)$ & C(14)-C(9)-P(1) & $119.9(3)$ \\
\hline O(3)-S-C(39) & $104.22(14)$ & $\mathrm{C}(11)-\mathrm{C}(10)-\mathrm{C}(9)$ & $120.7(3)$ \\
\hline O(2)-S-C(39) & $100.83(13)$ & $\mathrm{C}(11)-\mathrm{C}(10)-\mathrm{H}(10)$ & $120.2(19)$ \\
\hline $\mathrm{C}(1)-\mathrm{P}(1)-\mathrm{C}(3)$ & $111.06(15)$ & $\mathrm{C}(9)-\mathrm{C}(10)-\mathrm{H}(10)$ & 119.1(19) \\
\hline $\mathrm{C}(1)-\mathrm{P}(1)-\mathrm{C}(9)$ & $105.07(15)$ & $C(12)-C(11)-C(10)$ & $120.1(4)$ \\
\hline $\mathrm{C}(3)-\mathrm{P}(1)-\mathrm{C}(9)$ & $104.65(14)$ & $\mathrm{C}(12)-\mathrm{C}(11)-\mathrm{H}(11)$ & $120.7(19)$ \\
\hline $\mathrm{C}(1)-\mathrm{P}(1)-\mathrm{Pd}$ & $115.89(12)$ & $\mathrm{C}(10)-\mathrm{C}(11)-\mathrm{H}(11)$ & 119.1(19) \\
\hline $\mathrm{C}(3)-\mathrm{P}(1)-\mathrm{Pd}$ & $109.44(10)$ & $\mathrm{C}(13)-\mathrm{C}(12)-\mathrm{C}(11)$ & $120.3(4)$ \\
\hline $\mathrm{C}(9)-\mathrm{P}(1)-\mathrm{Pd}$ & $110.06(10)$ & $\mathrm{C}(13)-\mathrm{C}(12)-\mathrm{H}(12)$ & $123(2)$ \\
\hline $\mathrm{C}(2)-\mathrm{P}(2)-\mathrm{C}(15)$ & $111.07(15)$ & $\mathrm{C}(11)-\mathrm{C}(12)-\mathrm{H}(12)$ & $117(2)$ \\
\hline C(2)-P(2)-C(21) & $107.16(16)$ & $\mathrm{C}(12)-\mathrm{C}(13)-\mathrm{C}(14)$ & $120.1(4)$ \\
\hline $\mathrm{C}(15)-\mathrm{P}(2)-\mathrm{C}(21)$ & $104.93(14)$ & $\mathrm{C}(12)-\mathrm{C}(13)-\mathrm{H}(13)$ & $125.7(19)$ \\
\hline $\mathrm{C}(2)-\mathrm{P}(2)-\mathrm{Pd}$ & $116.48(12)$ & $\mathrm{C}(14)-\mathrm{C}(13)-\mathrm{H}(13)$ & 114.2(19) \\
\hline $\mathrm{C}(15)-\mathrm{P}(2)-\mathrm{Pd}$ & $109.51(11)$ & C(13)-C(14)-C(9) & $120.7(3)$ \\
\hline $\mathrm{C}(21)-\mathrm{P}(2)-\mathrm{Pd}$ & $106.95(10)$ & $\mathrm{C}(13)-\mathrm{C}(14)-\mathrm{H}(14)$ & $122.5(17)$ \\
\hline C(27)-B(1)-C(33) & $108.7(2)$ & $\mathrm{C}(9)-\mathrm{C}(14)-\mathrm{H}(14)$ & $116.8(18)$ \\
\hline C(27)-B(1)-C(1) & $110.4(3)$ & $C(16)-C(15)-C(20)$ & $118.6(3)$ \\
\hline $\mathrm{C}(33)-\mathrm{B}(1)-\mathrm{C}(1)$ & $108.3(3)$ & $\mathrm{C}(16)-\mathrm{C}(15)-\mathrm{P}(2)$ & $121.5(2)$ \\
\hline $\mathrm{C}(27)-\mathrm{B}(1)-\mathrm{C}(2)$ & $109.8(3)$ & $\mathrm{C}(20)-\mathrm{C}(15)-\mathrm{P}(2)$ & $119.8(3)$ \\
\hline $\mathrm{C}(33)-\mathrm{B}(1)-\mathrm{C}(2)$ & $110.1(3)$ & $C(17)-C(16)-C(15)$ & $121.2(4)$ \\
\hline $\mathrm{C}(1)-\mathrm{B}(1)-\mathrm{C}(2)$ & $109.5(3)$ & $\mathrm{C}(17)-\mathrm{C}(16)-\mathrm{H}(16)$ & $119.3(16)$ \\
\hline $\mathrm{S}-\mathrm{O}(2)-\mathrm{Pd}$ & $121.90(11)$ & $\mathrm{C}(15)-\mathrm{C}(16)-\mathrm{H}(16)$ & $119.2(15)$ \\
\hline $\mathrm{B}(1)-\mathrm{C}(1)-\mathrm{P}(1)$ & $120.1(2)$ & $\mathrm{C}(18)-\mathrm{C}(17)-\mathrm{C}(16)$ & $119.7(4)$ \\
\hline $\mathrm{B}(1)-\mathrm{C}(1)-\mathrm{H}(1 \mathrm{~A})$ & $111.2(16)$ & $\mathrm{C}(18)-\mathrm{C}(17)-\mathrm{H}(17)$ & $120.8(19)$ \\
\hline $\mathrm{P}(1)-\mathrm{C}(1)-\mathrm{H}(1 \mathrm{~A})$ & $100.5(15)$ & $\mathrm{C}(16)-\mathrm{C}(17)-\mathrm{H}(17)$ & $119(2)$ \\
\hline $\mathrm{B}(1)-\mathrm{C}(1)-\mathrm{H}(1 \mathrm{~B})$ & $114.7(18)$ & $\mathrm{C}(17)-\mathrm{C}(18)-\mathrm{C}(19)$ & $120.1(4)$ \\
\hline $\mathrm{P}(1)-\mathrm{C}(1)-\mathrm{H}(1 \mathrm{~B})$ & $105.2(18)$ & $\mathrm{C}(17)-\mathrm{C}(18)-\mathrm{H}(18)$ & $118.2(19)$ \\
\hline $\mathrm{H}(1 \mathrm{~A})-\mathrm{C}(1)-\mathrm{H}(1 \mathrm{~B})$ & $103(2)$ & $\mathrm{C}(19)-\mathrm{C}(18)-\mathrm{H}(18)$ & $121.7(19)$ \\
\hline $\mathrm{B}(1)-\mathrm{C}(2)-\mathrm{P}(2)$ & $117.2(2)$ & $\mathrm{C}(18)-\mathrm{C}(19)-\mathrm{C}(20)$ & $120.5(4)$ \\
\hline
\end{tabular}




\begin{tabular}{|c|c|c|c|}
\hline $\mathrm{C}(18)-\mathrm{C}(19)-\mathrm{H}(19)$ & $124(2)$ & $\mathrm{C}(36)-\mathrm{C}(37)-\mathrm{H}(37)$ & $125.5(19)$ \\
\hline $\mathrm{C}(20)-\mathrm{C}(19)-\mathrm{H}(19)$ & $116(2)$ & $\mathrm{C}(38)-\mathrm{C}(37)-\mathrm{H}(37)$ & $113.4(19)$ \\
\hline $\mathrm{C}(19)-\mathrm{C}(20)-\mathrm{C}(15)$ & $119.8(4)$ & $\mathrm{C}(37)-\mathrm{C}(38)-\mathrm{C}(33)$ & $122.3(4)$ \\
\hline $\mathrm{C}(19)-\mathrm{C}(20)-\mathrm{H}(20)$ & $121.8(18)$ & $\mathrm{C}(37)-\mathrm{C}(38)-\mathrm{H}(38)$ & $117.7(17)$ \\
\hline $\mathrm{C}(15)-\mathrm{C}(20)-\mathrm{H}(20)$ & $118.1(18)$ & $\mathrm{C}(33)-\mathrm{C}(38)-\mathrm{H}(38)$ & $120.0(17)$ \\
\hline $\mathrm{C}(22)-\mathrm{C}(21)-\mathrm{C}(26)$ & $118.6(3)$ & $\mathrm{F}(2)-\mathrm{C}(39)-\mathrm{F}(3)$ & $107.5(3)$ \\
\hline $\mathrm{C}(22)-\mathrm{C}(21)-\mathrm{P}(2)$ & $119.6(2)$ & $\mathrm{F}(2)-\mathrm{C}(39)-\mathrm{F}(1)$ & $107.7(3)$ \\
\hline $\mathrm{C}(26)-\mathrm{C}(21)-\mathrm{P}(2)$ & $121.8(3)$ & $\mathrm{F}(3)-\mathrm{C}(39)-\mathrm{F}(1)$ & $108.0(3)$ \\
\hline $\mathrm{C}(21)-\mathrm{C}(22)-\mathrm{C}(23)$ & $120.5(4)$ & $\mathrm{F}(2)-\mathrm{C}(39)-\mathrm{S}$ & $111.6(2)$ \\
\hline $\mathrm{C}(21)-\mathrm{C}(22)-\mathrm{H}(22)$ & $123.0(18)$ & $\mathrm{F}(3)-\mathrm{C}(39)-\mathrm{S}$ & $111.3(2)$ \\
\hline $\mathrm{C}(23)-\mathrm{C}(22)-\mathrm{H}(22)$ & $116.4(18)$ & $\mathrm{F}(1)-\mathrm{C}(39)-\mathrm{S}$ & $110.7(2)$ \\
\hline $\mathrm{C}(24)-\mathrm{C}(23)-\mathrm{C}(22)$ & $120.1(4)$ & $\mathrm{C}(43)-\mathrm{O}(1)-\mathrm{C}(40)$ & $109.9(2)$ \\
\hline $\mathrm{C}(24)-\mathrm{C}(23)-\mathrm{H}(23)$ & $118(2)$ & $\mathrm{C}(43)-\mathrm{O}(1)-\mathrm{Pd}$ & $114.92(17)$ \\
\hline $\mathrm{C}(22)-\mathrm{C}(23)-\mathrm{H}(23)$ & $122(2)$ & $\mathrm{C}(40)-\mathrm{O}(1)-\mathrm{Pd}$ & $133.80(17)$ \\
\hline$C(23)-C(24)-C(25)$ & $120.2(4)$ & $\mathrm{O}(1)-\mathrm{C}(40)-\mathrm{C}(41)$ & $104.7(2)$ \\
\hline $\mathrm{C}(23)-\mathrm{C}(24)-\mathrm{H}(24)$ & $119.7(17)$ & $\mathrm{O}(1)-\mathrm{C}(40)-\mathrm{H}(40 \mathrm{~A})$ & $105.6(17)$ \\
\hline $\mathrm{C}(25)-\mathrm{C}(24)-\mathrm{H}(24)$ & $120.0(17)$ & $\mathrm{C}(41)-\mathrm{C}(40)-\mathrm{H}(40 \mathrm{~A})$ & $113.8(17)$ \\
\hline$C(24)-C(25)-C(26)$ & $120.0(3)$ & $\mathrm{O}(1)-\mathrm{C}(40)-\mathrm{H}(40 \mathrm{~B})$ & $106.8(15)$ \\
\hline $\mathrm{C}(24)-\mathrm{C}(25)-\mathrm{H}(25)$ & $121.2(16)$ & $\mathrm{C}(41)-\mathrm{C}(40)-\mathrm{H}(40 \mathrm{~B})$ & $116.7(15)$ \\
\hline $\mathrm{C}(26)-\mathrm{C}(25)-\mathrm{H}(25)$ & $118.8(16)$ & $\mathrm{H}(40 \mathrm{~A})-\mathrm{C}(40)-\mathrm{H}(40 \mathrm{~B})$ & $108(2)$ \\
\hline$C(25)-C(26)-C(21)$ & $120.5(3)$ & $\mathrm{C}(40)-\mathrm{C}(41)-\mathrm{C}(42)$ & $101.0(3)$ \\
\hline $\mathrm{C}(25)-\mathrm{C}(26)-\mathrm{H}(26)$ & $118.1(17)$ & $\mathrm{C}(40)-\mathrm{C}(41)-\mathrm{H}(41 \mathrm{~A})$ & $110.8(16)$ \\
\hline $\mathrm{C}(21)-\mathrm{C}(26)-\mathrm{H}(26)$ & $121.4(17)$ & $\mathrm{C}(42)-\mathrm{C}(41)-\mathrm{H}(41 \mathrm{~A})$ & $107.1(16)$ \\
\hline $\mathrm{C}(28)-\mathrm{C}(27)-\mathrm{C}(32)$ & $115.2(3)$ & $\mathrm{C}(40)-\mathrm{C}(41)-\mathrm{H}(41 \mathrm{~B})$ & $115.1(16)$ \\
\hline $\mathrm{C}(28)-\mathrm{C}(27)-\mathrm{B}(1)$ & $124.3(3)$ & $\mathrm{C}(42)-\mathrm{C}(41)-\mathrm{H}(41 \mathrm{~B})$ & $110.8(16)$ \\
\hline $\mathrm{C}(32)-\mathrm{C}(27)-\mathrm{B}(1)$ & $120.5(3)$ & $\mathrm{H}(41 \mathrm{~A})-\mathrm{C}(41)-\mathrm{H}(41 \mathrm{~B})$ & $111(2)$ \\
\hline $\mathrm{C}(27)-\mathrm{C}(28)-\mathrm{C}(29)$ & $122.6(3)$ & $C(43)-C(42)-C(41)$ & $104.6(3)$ \\
\hline $\mathrm{C}(27)-\mathrm{C}(28)-\mathrm{H}(28)$ & $121.9(17)$ & $\mathrm{C}(43)-\mathrm{C}(42)-\mathrm{H}(42 \mathrm{~A})$ & $112.4(18)$ \\
\hline $\mathrm{C}(29)-\mathrm{C}(28)-\mathrm{H}(28)$ & $115.5(17)$ & $\mathrm{C}(41)-\mathrm{C}(42)-\mathrm{H}(42 \mathrm{~A})$ & $115.3(18)$ \\
\hline $\mathrm{C}(30)-\mathrm{C}(29)-\mathrm{C}(28)$ & $119.9(4)$ & $\mathrm{C}(43)-\mathrm{C}(42)-\mathrm{H}(42 \mathrm{~B})$ & $108.4(17)$ \\
\hline $\mathrm{C}(30)-\mathrm{C}(29)-\mathrm{H}(29)$ & $121.2(17)$ & $\mathrm{C}(41)-\mathrm{C}(42)-\mathrm{H}(42 \mathrm{~B})$ & $110.1(17)$ \\
\hline $\mathrm{C}(28)-\mathrm{C}(29)-\mathrm{H}(29)$ & $118.9(17)$ & $\mathrm{H}(42 \mathrm{~A})-\mathrm{C}(42)-\mathrm{H}(42 \mathrm{~B})$ & $106(2)$ \\
\hline$C(29)-C(30)-C(31)$ & $119.2(3)$ & $\mathrm{O}(1)-\mathrm{C}(43)-\mathrm{C}(42)$ & $104.3(3)$ \\
\hline $\mathrm{C}(29)-\mathrm{C}(30)-\mathrm{H}(30)$ & $123.6(18)$ & $\mathrm{O}(1)-\mathrm{C}(43)-\mathrm{H}(43 \mathrm{~A})$ & $109.7(19)$ \\
\hline $\mathrm{C}(31)-\mathrm{C}(30)-\mathrm{H}(30)$ & $117.2(18)$ & $\mathrm{C}(42)-\mathrm{C}(43)-\mathrm{H}(43 \mathrm{~A})$ & $111.3(19)$ \\
\hline$C(32)-C(31)-C(30)$ & $120.0(4)$ & $\mathrm{O}(1)-\mathrm{C}(43)-\mathrm{H}(43 \mathrm{~B})$ & $106.8(16)$ \\
\hline $\mathrm{C}(32)-\mathrm{C}(31)-\mathrm{H}(31)$ & $115(2)$ & $\mathrm{C}(42)-\mathrm{C}(43)-\mathrm{H}(43 \mathrm{~B})$ & 113.1(16) \\
\hline $\mathrm{C}(30)-\mathrm{C}(31)-\mathrm{H}(31)$ & $124(2)$ & $\mathrm{H}(43 \mathrm{~A})-\mathrm{C}(43)-\mathrm{H}(43 \mathrm{~B})$ & 111(2) \\
\hline $\mathrm{C}(31)-\mathrm{C}(32)-\mathrm{C}(27)$ & 123.1(3) & $\mathrm{C}(54)-\mathrm{O}(5)-\mathrm{C}(51)$ & $108.4(5)$ \\
\hline $\mathrm{C}(31)-\mathrm{C}(32)-\mathrm{H}(32)$ & $118.8(16)$ & $\mathrm{C}(52)-\mathrm{C}(51)-\mathrm{O}(5)$ & $106.3(4)$ \\
\hline $\mathrm{C}(27)-\mathrm{C}(32)-\mathrm{H}(32)$ & $117.9(16)$ & $\mathrm{C}(52)-\mathrm{C}(51)-\mathrm{H}(51 \mathrm{~A})$ & 110.5 \\
\hline $\mathrm{C}(34)-\mathrm{C}(33)-\mathrm{C}(38)$ & $114.7(3)$ & $\mathrm{O}(5)-\mathrm{C}(51)-\mathrm{H}(51 \mathrm{~A})$ & 110.5 \\
\hline $\mathrm{C}(34)-\mathrm{C}(33)-\mathrm{B}(1)$ & $124.0(3)$ & $\mathrm{C}(52)-\mathrm{C}(51)-\mathrm{H}(51 \mathrm{~B})$ & 110.5 \\
\hline $\mathrm{C}(38)-\mathrm{C}(33)-\mathrm{B}(1)$ & 121.3(3) & $\mathrm{O}(5)-\mathrm{C}(51)-\mathrm{H}(51 \mathrm{~B})$ & 110.5 \\
\hline $\mathrm{C}(33)-\mathrm{C}(34)-\mathrm{C}(35)$ & $122.7(4)$ & $\mathrm{H}(51 \mathrm{~A})-\mathrm{C}(51)-\mathrm{H}(51 \mathrm{~B})$ & 108.7 \\
\hline $\mathrm{C}(33)-\mathrm{C}(34)-\mathrm{H}(34)$ & $121.8(18)$ & $C(51)-C(52)-C(53)$ & $101.3(5)$ \\
\hline $\mathrm{C}(35)-\mathrm{C}(34)-\mathrm{H}(34)$ & $115.4(18)$ & $\mathrm{C}(51)-\mathrm{C}(52)-\mathrm{H}(52 \mathrm{~A})$ & 111.5 \\
\hline$C(36)-C(35)-C(34)$ & $120.9(4)$ & $\mathrm{C}(53)-\mathrm{C}(52)-\mathrm{H}(52 \mathrm{~A})$ & 111.5 \\
\hline $\mathrm{C}(36)-\mathrm{C}(35)-\mathrm{H}(35)$ & $124(2)$ & $\mathrm{C}(51)-\mathrm{C}(52)-\mathrm{H}(52 \mathrm{~B})$ & 111.5 \\
\hline $\mathrm{C}(34)-\mathrm{C}(35)-\mathrm{H}(35)$ & $116(2)$ & $\mathrm{C}(53)-\mathrm{C}(52)-\mathrm{H}(52 \mathrm{~B})$ & 111.5 \\
\hline$C(35)-C(36)-C(37)$ & $118.6(4)$ & $\mathrm{H}(52 \mathrm{~A})-\mathrm{C}(52)-\mathrm{H}(52 \mathrm{~B})$ & 109.3 \\
\hline $\mathrm{C}(35)-\mathrm{C}(36)-\mathrm{H}(36)$ & $118.9(18)$ & $\mathrm{C}(54)-\mathrm{C}(53)-\mathrm{C}(52)$ & $114.2(4)$ \\
\hline $\mathrm{C}(37)-\mathrm{C}(36)-\mathrm{H}(36)$ & $122.3(18)$ & $\mathrm{C}(54)-\mathrm{C}(53)-\mathrm{H}(53 \mathrm{~A})$ & 108.7 \\
\hline $\mathrm{C}(36)-\mathrm{C}(37)-\mathrm{C}(38)$ & $120.7(4)$ & $\mathrm{C}(52)-\mathrm{C}(53)-\mathrm{H}(53 \mathrm{~A})$ & 108.7 \\
\hline
\end{tabular}


$\mathrm{C}(54)-\mathrm{C}(53)-\mathrm{H}(53 \mathrm{~B})$

$\mathrm{C}(52)-\mathrm{C}(53)-\mathrm{H}(53 \mathrm{~B})$

$\mathrm{H}(53 \mathrm{~A})-\mathrm{C}(53)-\mathrm{H}(53 \mathrm{~B})$

$\mathrm{O}(5)-\mathrm{C}(54)-\mathrm{C}(53)$

$\mathrm{O}(5)-\mathrm{C}(54)-\mathrm{H}(54 \mathrm{~A})$
108.7

108.7

107.6

$106.0(5)$

110.5
$\mathrm{C}(53)-\mathrm{C}(54)-\mathrm{H}(54 \mathrm{~A})$

$\mathrm{O}(5)-\mathrm{C}(54)-\mathrm{H}(54 \mathrm{~B})$

$\mathrm{C}(53)-\mathrm{C}(54)-\mathrm{H}(54 \mathrm{~B})$

$\mathrm{H}(54 \mathrm{~A})-\mathrm{C}(54)-\mathrm{H}(54 \mathrm{~B})$
110.5

110.5

110.5

108.7 
Table 4. Anisotropic displacement parameters $\left(\AA^{2} \times 1^{4}\right)$ for $1^{\prime}$ (CCDC 171286). The anisotropic displacement factor exponent takes the form: $-2 \pi^{2}\left[h^{2} a^{* 2} U 11+\ldots+2 h k a^{*} b^{*}\right.$ $\mathbf{U}^{12}$ ]

\begin{tabular}{|c|c|c|c|c|c|c|}
\hline & $\mathrm{U}^{11}$ & $\mathrm{U}^{22}$ & $\mathrm{U}^{33}$ & $\mathrm{U}^{23}$ & $\mathrm{U}^{13}$ & $\mathrm{U}^{12}$ \\
\hline $\mathrm{Pd}$ & $174(1)$ & $252(1)$ & $206(1)$ & $45(1)$ & $38(1)$ & $0(1)$ \\
\hline $\mathrm{S}$ & $242(5)$ & $311(5)$ & $247(5)$ & $-23(4)$ & $38(4)$ & $-14(4)$ \\
\hline $\mathrm{P}(1)$ & $188(4)$ & $257(5)$ & $218(5)$ & $38(4)$ & $50(4)$ & $25(4)$ \\
\hline $\mathrm{P}(2)$ & $186(5)$ & $292(5)$ & $238(5)$ & $68(4)$ & $24(4)$ & $-9(4)$ \\
\hline $\mathrm{B}(1)$ & 174(19) & $300(20)$ & $280(20)$ & $72(17)$ & $35(16)$ & $7(16)$ \\
\hline$F(1)$ & $363(12)$ & 571(14) & $305(11)$ & $125(10)$ & $2(9)$ & $67(10)$ \\
\hline$F(2)$ & $394(12)$ & $425(13)$ & $500(13)$ & $-174(11)$ & $-61(10)$ & $21(10)$ \\
\hline $\mathrm{F}(3)$ & $216(11)$ & $522(13)$ & $444(12)$ & $43(10)$ & $41(9)$ & $-32(9)$ \\
\hline $\mathrm{O}(2)$ & $219(12)$ & $305(13)$ & $196(12)$ & $4(10)$ & $73(9)$ & $-31(10)$ \\
\hline $\mathrm{O}(3)$ & $334(13)$ & 298(13) & $348(13)$ & $-99(11)$ & $46(10)$ & $75(11)$ \\
\hline $\mathrm{O}(4)$ & $340(14)$ & $320(13)$ & $233(12)$ & $104(10)$ & $70(10)$ & $2(11)$ \\
\hline$C(1)$ & $218(19)$ & $310(20)$ & $260(20)$ & $91(17)$ & $106(16)$ & $65(16)$ \\
\hline$C(2)$ & $210(20)$ & $300(20)$ & $290(20)$ & $63(17)$ & $-6(16)$ & $-48(17)$ \\
\hline$C(3)$ & $215(17)$ & 241(19) & $215(17)$ & $26(14)$ & $10(14)$ & 1(14) \\
\hline$C(4)$ & $380(20)$ & $340(20)$ & $310(20)$ & $96(18)$ & $104(17)$ & $132(18)$ \\
\hline$C(5)$ & $400(20)$ & $460(30)$ & $500(30)$ & $170(20)$ & $230(20)$ & $230(20)$ \\
\hline$C(6)$ & $330(20)$ & $380(20)$ & $460(30)$ & 171(19) & $63(19)$ & $186(19)$ \\
\hline$C(7)$ & $310(20)$ & $410(20)$ & $360(20)$ & $178(18)$ & $33(18)$ & $70(18)$ \\
\hline$C(8)$ & 221(19) & $390(20)$ & $290(20)$ & $80(16)$ & $49(16)$ & $78(16)$ \\
\hline$C(9)$ & $260(19)$ & 251(19) & $217(18)$ & $38(14)$ & $104(15)$ & $15(15)$ \\
\hline$C(10)$ & $320(20)$ & $350(20)$ & $350(20)$ & $-87(18)$ & $67(18)$ & $59(18)$ \\
\hline$C(11)$ & $380(30)$ & $440(30)$ & $400(20)$ & $-140(19)$ & $30(20)$ & $-10(20)$ \\
\hline$C(12)$ & $580(30)$ & $330(20)$ & $340(20)$ & $-123(19)$ & $140(20)$ & $-10(20)$ \\
\hline$C(13)$ & $440(30)$ & $290(20)$ & $390(20)$ & $18(18)$ & $190(20)$ & $100(20)$ \\
\hline$C(14)$ & $300(20)$ & $330(20)$ & $310(20)$ & $86(17)$ & $112(17)$ & $46(18)$ \\
\hline$C(15)$ & 193(18) & $380(20)$ & 233(18) & $106(15)$ & $40(14)$ & $-22(15)$ \\
\hline$C(16)$ & $240(20)$ & $420(20)$ & 233(19) & $62(17)$ & $43(16)$ & $14(18)$ \\
\hline$C(17)$ & $370(20)$ & $450(30)$ & $310(20)$ & $110(20)$ & $103(18)$ & $150(20)$ \\
\hline$C(18)$ & $310(20)$ & $650(30)$ & $450(30)$ & $240(20)$ & $110(20)$ & $150(20)$ \\
\hline C(19) & $210(20)$ & $710(40)$ & $460(30)$ & $250(20)$ & $-39(18)$ & $-90(20)$ \\
\hline$C(20)$ & $280(20)$ & $420(30)$ & $410(20)$ & $140(20)$ & $17(18)$ & $-43(19)$ \\
\hline$C(21)$ & $200(17)$ & $310(20)$ & $269(18)$ & $31(16)$ & $34(14)$ & $-77(16)$ \\
\hline$C(22)$ & $410(20)$ & $510(30)$ & $260(20)$ & $80(20)$ & $22(18)$ & $80(20)$ \\
\hline$C(23)$ & $520(30)$ & $490(30)$ & $420(30)$ & $-10(20)$ & $60(20)$ & $200(20)$ \\
\hline$C(24)$ & $420(20)$ & $500(30)$ & $290(20)$ & $-90(20)$ & $39(19)$ & $-50(20)$ \\
\hline$C(25)$ & $320(20)$ & $430(20)$ & 231(19) & $-11(19)$ & $-55(16)$ & $-99(19)$ \\
\hline$C(26)$ & $250(20)$ & $280(20)$ & $320(20)$ & $18(17)$ & $-27(16)$ & $-90(16)$ \\
\hline$C(27)$ & $154(17)$ & $300(20)$ & 278(19) & $55(15)$ & $41(14)$ & $-39(15)$ \\
\hline$C(28)$ & $270(20)$ & $360(20)$ & $260(20)$ & $52(17)$ & $33(16)$ & $-20(16)$ \\
\hline$C(29)$ & $310(20)$ & $300(20)$ & $380(20)$ & $77(18)$ & $60(17)$ & $43(17)$ \\
\hline $\mathrm{C}(30)$ & $300(20)$ & $410(20)$ & $300(20)$ & $187(19)$ & $-4(17)$ & $-3(18)$ \\
\hline $\mathrm{C}(31)$ & $280(20)$ & $470(30)$ & $250(20)$ & $60(19)$ & 81(17) & $-41(18)$ \\
\hline $\mathrm{C}(32)$ & $227(19)$ & $300(20)$ & $320(20)$ & $46(18)$ & $88(15)$ & 11(16) \\
\hline$C(33)$ & $245(18)$ & $320(20)$ & 263(18) & $157(17)$ & $64(14)$ & $-3(17)$ \\
\hline$C(34)$ & $250(20)$ & $460(30)$ & $360(20)$ & $82(19)$ & $63(18)$ & $8(19)$ \\
\hline$C(35)$ & $250(20)$ & $540(30)$ & $550(30)$ & $140(20)$ & 130(20) & $90(20)$ \\
\hline$C(36)$ & 190(20) & $570(30)$ & $600(30)$ & $300(20)$ & $30(20)$ & $0(20)$ \\
\hline
\end{tabular}




\begin{tabular}{lrcrrrr}
$\mathrm{C}(37)$ & $310(20)$ & $480(30)$ & $520(30)$ & $230(20)$ & $-40(20)$ & $-170(20)$ \\
$\mathrm{C}(38)$ & $310(20)$ & $320(20)$ & $530(30)$ & $140(20)$ & $48(19)$ & $-27(19)$ \\
$\mathrm{C}(39)$ & $330(20)$ & $310(20)$ & $260(20)$ & $25(16)$ & $39(16)$ & $45(17)$ \\
$\mathrm{O}(1)$ & $261(12)$ & $244(12)$ & $199(12)$ & $46(9)$ & $62(9)$ & $46(10)$ \\
$\mathrm{C}(40)$ & $233(19)$ & $310(20)$ & $239(18)$ & $-16(16)$ & $68(15)$ & $0(17)$ \\
$\mathrm{C}(41)$ & $340(20)$ & $320(20)$ & $260(20)$ & $57(16)$ & $85(18)$ & $12(17)$ \\
$\mathrm{C}(42)$ & $320(20)$ & $310(20)$ & $350(20)$ & $94(18)$ & $74(18)$ & $55(19)$ \\
$\mathrm{C}(43)$ & $320(20)$ & $260(20)$ & $300(20)$ & $68(16)$ & $47(18)$ & $56(17)$ \\
& & & & & \\
$\mathrm{O}(5)$ & $1050(40)$ & $1310(40)$ & $2660(70)$ & $1090(40)$ & $-540(40)$ & $-360(30)$ \\
$\mathrm{C}(51)$ & $1620(80)$ & $3660(140)$ & $880(50)$ & $910(70)$ & $860(60)$ & $630(90)$ \\
$\mathrm{C}(52)$ & $790(50)$ & $2130(90)$ & $1960(80)$ & $1450(70)$ & $120(50)$ & $-110(50)$ \\
$\mathrm{C}(53)$ & $890(50)$ & $500(40)$ & $2240(80)$ & $330(40)$ & $310(50)$ & $-90(30)$ \\
$\mathrm{C}(54)$ & $1480(70)$ & $1360(60)$ & $820(40)$ & $540(40)$ & $-220(40)$ & $-690(50)$ \\
& & & & & \\
\hline
\end{tabular}




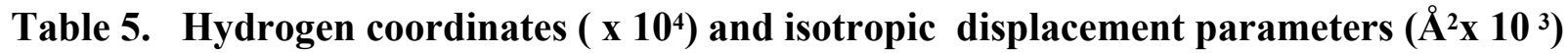
for 1' (CCDC 171286).

\begin{tabular}{|c|c|c|c|c|}
\hline & $\mathrm{x}$ & $\mathrm{y}$ & $\mathrm{Z}$ & $\mathrm{U}_{\text {iso }}$ \\
\hline $\mathrm{H}(1 \mathrm{~A})$ & $7019(18)$ & $2023(15)$ & $8299(12)$ & $14(7)$ \\
\hline $\mathrm{H}(1 \mathrm{~B})$ & $6630(20)$ & $2388(16)$ & $7638(15)$ & $34(9)$ \\
\hline $\mathrm{H}(2 \mathrm{~A})$ & $6140(20)$ & $3702(15)$ & $9090(13)$ & $23(8)$ \\
\hline $\mathrm{H}(2 \mathrm{~B})$ & $7190(20)$ & $3815(15)$ & $9422(13)$ & $17(8)$ \\
\hline $\mathrm{H}(4)$ & $3680(20)$ & $2873(17)$ & $8876(16)$ & $47(10)$ \\
\hline $\mathrm{H}(5)$ & $2470(20)$ & $3752(18)$ & $8396(16)$ & $42(11)$ \\
\hline $\mathrm{H}(6)$ & $2620(20)$ & $4340(17)$ & $7443(16)$ & $35(10)$ \\
\hline $\mathrm{H}(7)$ & $3930(20)$ & $4143(17)$ & $6730(16)$ & $44(10)$ \\
\hline $\mathrm{H}(8)$ & $5130(20)$ & $3219(15)$ & $7160(14)$ & $28(9)$ \\
\hline $\mathrm{H}(10)$ & $3620(20)$ & $1685(15)$ & $7525(14)$ & $20(9)$ \\
\hline $\mathrm{H}(11)$ & $3190(20)$ & $700(16)$ & $6797(14)$ & $24(9)$ \\
\hline $\mathrm{H}(12)$ & $4340(20)$ & $-187(18)$ & $6606(16)$ & $42(11)$ \\
\hline $\mathrm{H}(13)$ & $5990(20)$ & $-91(16)$ & $7173(14)$ & $28(9)$ \\
\hline $\mathrm{H}(14)$ & $6410(20)$ & $891(16)$ & $7912(14)$ & $27(9)$ \\
\hline $\mathrm{H}(16)$ & 7171(19) & $1142(14)$ & $9546(13)$ & $13(7)$ \\
\hline $\mathrm{H}(17)$ & $8610(20)$ & $425(17)$ & $9869(15)$ & $31(10)$ \\
\hline $\mathrm{H}(18)$ & $10080(20)$ & $976(18)$ & $10356(15)$ & $44(10)$ \\
\hline $\mathrm{H}(19)$ & $10020(20)$ & $2301(17)$ & $10617(15)$ & $32(10)$ \\
\hline $\mathrm{H}(20)$ & $8590(20)$ & 2987(16) & $10388(13)$ & $19(9)$ \\
\hline $\mathrm{H}(22)$ & $5600(20)$ & $4082(16)$ & $10196(15)$ & $29(9)$ \\
\hline $\mathrm{H}(23)$ & $5350(20)$ & $4575(18)$ & $11255(16)$ & $44(12)$ \\
\hline $\mathrm{H}(24)$ & $5900(20)$ & $4055(15)$ & $12292(14)$ & $26(8)$ \\
\hline $\mathrm{H}(25)$ & $6775(19)$ & $2878(15)$ & $12294(14)$ & $23(8)$ \\
\hline $\mathrm{H}(26)$ & $7090(20)$ & $2348(15)$ & $11249(13)$ & $18(8)$ \\
\hline $\mathrm{H}(28)$ & $6420(20)$ & $4778(16)$ & $8505(15)$ & $36(9)$ \\
\hline $\mathrm{H}(29)$ & $6130(20)$ & $5710(16)$ & $7669(14)$ & $23(8)$ \\
\hline $\mathrm{H}(30)$ & $6490(20)$ & $5469(16)$ & $6506(14)$ & $25(9)$ \\
\hline $\mathrm{H}(31)$ & $7140(20)$ & $4304(16)$ & $6259(15)$ & $23(9)$ \\
\hline $\mathrm{H}(32)$ & $7430(18)$ & $3406(14)$ & $7037(12)$ & $6(7)$ \\
\hline $\mathrm{H}(34)$ & $8560(20)$ & 2111(16) & $7999(14)$ & $25(9)$ \\
\hline $\mathrm{H}(35)$ & $10180(20)$ & $1906(17)$ & $8209(16)$ & $38(11)$ \\
\hline $\mathrm{H}(36)$ & $11260(20)$ & $2702(16)$ & $8921(15)$ & $35(9)$ \\
\hline $\mathrm{H}(37)$ & $10470(20)$ & $3835(17)$ & $9378(16)$ & $37(10)$ \\
\hline $\mathrm{H}(38)$ & $8835(19)$ & $4038(14)$ & $9108(13)$ & $9(8)$ \\
\hline $\mathrm{H}(40 \mathrm{~A})$ & $3950(20)$ & $1781(15)$ & $10937(14)$ & $29(9)$ \\
\hline $\mathrm{H}(40 \mathrm{~B})$ & $4780(20)$ & $2416(16)$ & $11166(13)$ & $25(9)$ \\
\hline $\mathrm{H}(41 \mathrm{~A})$ & $5670(20)$ & $1523(16)$ & $11963(14)$ & $33(9)$ \\
\hline $\mathrm{H}(41 \mathrm{~B})$ & $4480(20)$ & $1260(15)$ & $12067(14)$ & $29(8)$ \\
\hline $\mathrm{H}(42 \mathrm{~A})$ & $5570(20)$ & $252(16)$ & $11613(14)$ & $24(9)$ \\
\hline $\mathrm{H}(42 \mathrm{~B})$ & $4540(20)$ & $340(16)$ & $11203(14)$ & $28(9)$ \\
\hline $\mathrm{H}(43 \mathrm{~A})$ & $6360(20)$ & $885(17)$ & $10806(16)$ & $42(10)$ \\
\hline $\mathrm{H}(43 \mathrm{~B})$ & $5460(20)$ & $480(16)$ & $10278(15)$ & $39(9)$ \\
\hline $\mathrm{H}(51 \mathrm{~A})$ & 5824 & 1012 & 5628 & 240 \\
\hline $\mathrm{H}(51 \mathrm{~B})$ & 6603 & 599 & 6206 & 240 \\
\hline $\mathrm{H}(52 \mathrm{~A})$ & 7311 & 1732 & 6348 & 195 \\
\hline $\mathrm{H}(52 \mathrm{~B})$ & 6789 & 2035 & 5603 & 195 \\
\hline $\mathrm{H}(53 \mathrm{~A})$ & 8247 & 1813 & 5219 & 144 \\
\hline
\end{tabular}


$\mathrm{H}(53 \mathrm{~B})$

$\mathrm{H}(54 \mathrm{~A})$

H(54B)
8626

8013

8469
1308

825

324
5889

4604

5265
144

149

149 
Figure 2. Labeled drawing of 5 (CCDC 172948), with 50\% ellipsoids. Hydrogen atoms and solvent molecules have been omitted for clarity.

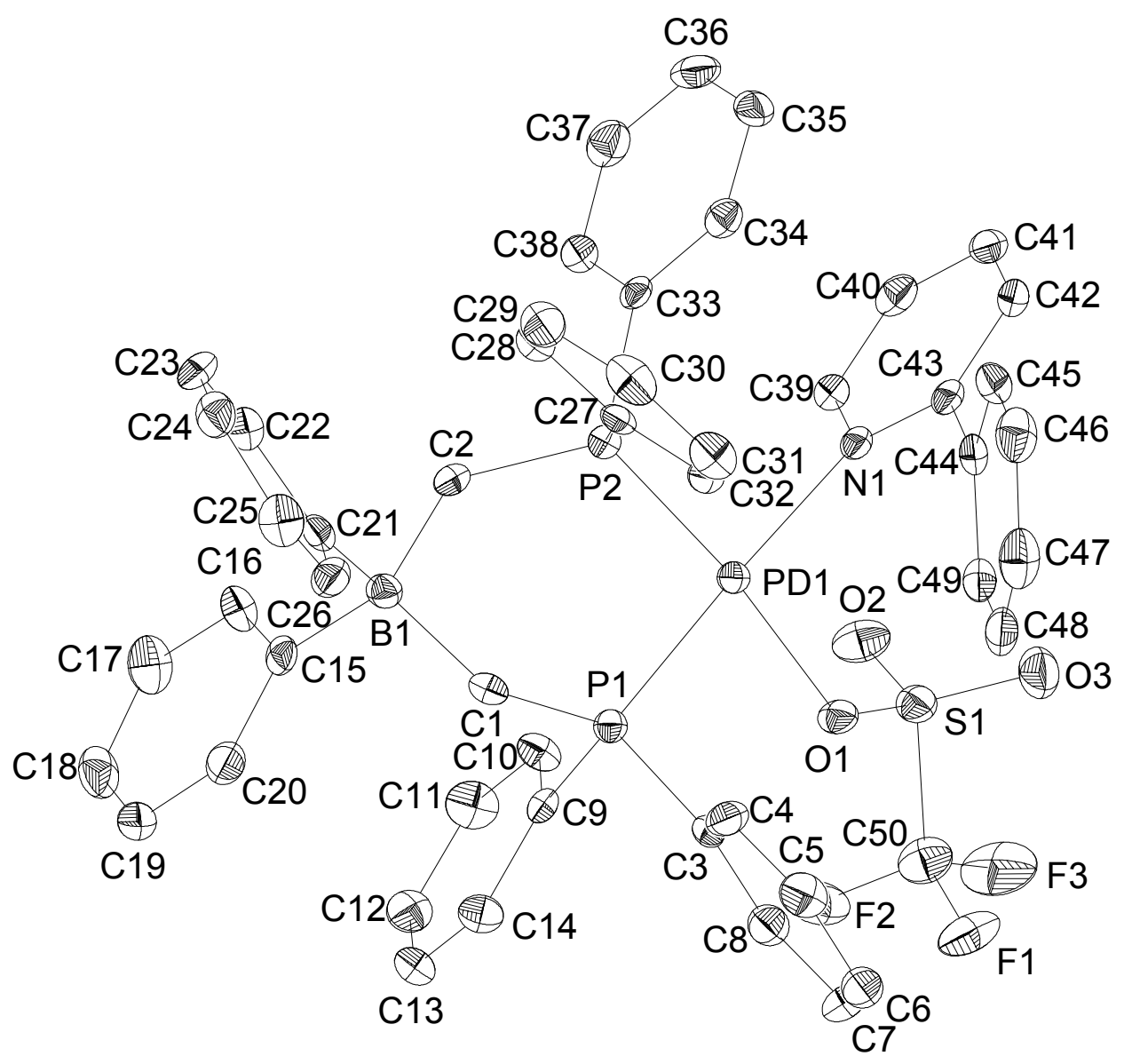


Table 6. Crystal data and structure refinement for 5 (CCDC 172948).

Empirical formula

Formula weight

Crystallization Solvent

Crystal Habit

Crystal size

Crystal color

Preliminary Photos

Type of diffractometer

Wavelength

Data Collection Temperature

$\theta$ range for 25758 reflections used in lattice determination

Unit cell dimensions

Volume

Z

Crystal system

Space group

Density (calculated)

$\mathrm{F}(000)$

Data collection program

$\theta$ range for data collection

Completeness to $\theta=28.42^{\circ}$

Index ranges

Data collection scan type

Data reduction program

Reflections collected

Independent reflections

Absorption coefficient

Absorption correction

Max. and min. transmission
$\mathrm{C}_{50} \mathrm{H}_{43} \mathrm{BF}_{3} \mathrm{NO}_{3} \mathrm{P}_{2} \mathrm{PdS} \cdot 2\left(\mathrm{CH}_{2} \mathrm{Cl}_{2}\right)$

1143.92

Dichloromethane/petroleum ether

Plate

$0.29 \times 0.28 \times 0.05 \mathrm{~mm}^{3}$

Dichroic Pink/Yellow

\section{Data Collection}

Rotation

CCD area detector

$0.71073 \AA \mathrm{MoK} \alpha$

98(2) K

2.75 to $25.50^{\circ}$

$\mathrm{a}=16.3584(11) \AA$

$\mathrm{b}=17.4150(12) \AA$

$\mathrm{c}=19.5418(13) \AA$

$\beta=112.1880(10)^{\circ}$

5154.9(6) $\AA^{3}$

4

Monoclinic

$\mathrm{P} 2{ }_{1} / \mathrm{n}$

$1.474 \mathrm{Mg} / \mathrm{m}^{3}$

2328

Bruker SMART

1.39 to $28.42^{\circ}$

$94.8 \%$

$-21 \leq \mathrm{h} \leq 21,-23 \leq \mathrm{k} \leq 23,-25 \leq 1 \leq 25$

$\omega$ scans at $7 \phi$ settings

Bruker SAINT v6.2

104619

$12279\left[\mathrm{R}_{\mathrm{int}}=0.0830\right]$

$0.724 \mathrm{~mm}^{-1}$

None

0.9661 and 0.8186 


\section{Table 1 (cont.)}

\section{Structure solution and Refinement}

Structure solution program

Primary solution method

Secondary solution method

Hydrogen placement

Structure refinement program

Refinement method

Data / restraints / parameters

Treatment of hydrogen atoms

Goodness-of-fit on $\mathrm{F}^{2}$

Final $\mathrm{R}$ indices $[\mathrm{I}>2 \sigma(\mathrm{I}), 8471$ reflections $]$

$\mathrm{R}$ indices (all data)

Type of weighting scheme used

Weighting scheme used

Max shift/error

Average shift/error

Largest diff. peak and hole
SHELXS-97 (Sheldrick, 1990)

Patterson method

Difference Fourier map

Difference Fourier map

SHELXL-97 (Sheldrick, 1997)

Full matrix least-squares on $\mathrm{F}^{2}$

$12279 / 0 / 785$

Unrestrained except for solvent

1.940

$\mathrm{R} 1=0.0489, w \mathrm{R} 2=0.0802$

$\mathrm{R} 1=0.0820, w \mathrm{R} 2=0.0830$

Sigma

$w=1 / \sigma^{2}\left(\mathrm{Fo}^{2}\right)$

0.106

0.000

1.648 and -1.247 e. $\AA^{-3}$

\section{Special Refinement Details}

Refinement of $\mathrm{F}^{2}$ against ALL reflections. The weighted R-factor $(w \mathrm{R})$ and goodness of fit $(\mathrm{S})$ are based on $\mathrm{F}^{2}$, conventional $\mathrm{R}$-factors $(\mathrm{R})$ are based on $\mathrm{F}$, with $\mathrm{F}$ set to zero for negative $\mathrm{F}^{2}$. The threshold expression of $\mathrm{F}^{2}>$ $2 \sigma\left(\mathrm{F}^{2}\right)$ is used only for calculating R-factors $(\mathrm{gt})$ etc. and is not relevant to the choice of reflections for refinement. $\mathrm{R}$-factors based on $\mathrm{F}^{2}$ are statistically about twice as large as those based on $\mathrm{F}$, and R-factors based on ALL data will be even larger.

All esds (except the esd in the dihedral angle between two 1.s. planes) are estimated using the full covariance matrix. The cell esds are taken into account individually in the estimation of esds in distances, angles and torsion angles; correlations between esds in cell parameters are only used when they are defined by crystal symmetry. An approximate (isotropic) treatment of cell esds is used for estimating esds involving l.s. planes. 
Table 7. Atomic coordinates $\left(x^{10}\right)$ and equivalent isotropic displacement parameters $\left(\AA^{2} \times 1^{3}\right)$ for 5 (CCDC 172948). U(eq) is defined as the trace of the orthogonalized $U^{i j}$ tensor.

\begin{tabular}{|c|c|c|c|c|}
\hline & $\mathrm{x}$ & $\mathrm{y}$ & $\mathrm{z}$ & $\mathrm{U}_{\mathrm{eq}}$ \\
\hline$\overline{\mathrm{Pd}(1)}$ & $5298(1)$ & 1351(1) & $7735(1)$ & $15(1)$ \\
\hline $\mathrm{S}(1)$ & $4153(1)$ & $-254(1)$ & $7139(1)$ & $21(1)$ \\
\hline $\mathrm{P}(1)$ & $4770(1)$ & $2217(1)$ & 6801(1) & $15(1)$ \\
\hline $\mathrm{P}(2)$ & 6489(1) & $2108(1)$ & $8250(1)$ & $16(1)$ \\
\hline $\mathrm{F}(1)$ & $2516(1)$ & $-92(1)$ & $6187(1)$ & $39(1)$ \\
\hline $\mathrm{F}(2)$ & $3469(1)$ & $-263(1)$ & $5688(1)$ & $40(1)$ \\
\hline $\mathrm{F}(3)$ & $3079(2)$ & $-1217(1)$ & 6201(1) & $52(1)$ \\
\hline $\mathrm{N}(1)$ & $5737(2)$ & $518(2)$ & $8603(1)$ & $15(1)$ \\
\hline $\mathrm{O}(1)$ & $4207(1)$ & $588(1)$ & 7099(1) & $20(1)$ \\
\hline $\mathrm{O}(2)$ & 4899(2) & $-654(1)$ & $7095(1)$ & $30(1)$ \\
\hline $\mathrm{O}(3)$ & $3824(2)$ & $-511(1)$ & $7685(1)$ & $33(1)$ \\
\hline $\mathrm{B}(1)$ & 6372(3) & $3141(2)$ & 7001(2) & $17(1)$ \\
\hline $\mathrm{C}(1)$ & $5305(2)$ & $3128(2)$ & 6884(2) & $17(1)$ \\
\hline$C(2)$ & 6906(2) & $2423(2)$ & $7573(2)$ & $18(1)$ \\
\hline$C(3)$ & $3633(2)$ & $2401(2)$ & $6702(2)$ & $15(1)$ \\
\hline$C(4)$ & $3439(2)$ & $3008(2)$ & 7089(2) & $21(1)$ \\
\hline$C(5)$ & 2589(3) & $3148(2)$ & $7037(2)$ & $26(1)$ \\
\hline$C(6)$ & 1902(3) & $2690(2)$ & $6594(2)$ & $27(1)$ \\
\hline$C(7)$ & 2074(3) & 2091(2) & 6203(2) & $27(1)$ \\
\hline $\mathrm{C}(8)$ & 2932(2) & $1944(2)$ & $6258(2)$ & $22(1)$ \\
\hline C(9) & 4751(2) & $1803(2)$ & $5945(2)$ & $15(1)$ \\
\hline$C(10)$ & $5267(2)$ & $1165(2)$ & $5960(2)$ & $21(1)$ \\
\hline $\mathrm{C}(11)$ & 5353(3) & $911(2)$ & $5314(2)$ & $27(1)$ \\
\hline$C(12)$ & 4924(2) & $1290(2)$ & $4653(2)$ & $24(1)$ \\
\hline$C(13)$ & 4394(2) & 1920(2) & 4633(2) & $23(1)$ \\
\hline$C(14)$ & $4309(2)$ & $2176(2)$ & $5275(2)$ & $20(1)$ \\
\hline$C(15)$ & $6512(2)$ & $3057(2)$ & $6218(2)$ & $17(1)$ \\
\hline$C(16)$ & $7129(3)$ & $2571(2)$ & $6105(2)$ & $23(1)$ \\
\hline$C(17)$ & 7243(3) & $2517(2)$ & $5433(2)$ & $30(1)$ \\
\hline$C(18)$ & 6734(3) & $2957(2)$ & $4835(2)$ & $29(1)$ \\
\hline$C(19)$ & 6123(3) & $3452(2)$ & 4923(2) & $27(1)$ \\
\hline$C(20)$ & $6022(2)$ & $3501(2)$ & $5602(2)$ & $23(1)$ \\
\hline$C(21)$ & $6786(2)$ & $3964(2)$ & $7383(2)$ & $17(1)$ \\
\hline$C(22)$ & 7706(2) & $4082(2)$ & 7695(2) & $21(1)$ \\
\hline$C(23)$ & $8084(3)$ & $4742(2)$ & $8079(2)$ & $24(1)$ \\
\hline$C(24)$ & 7567(3) & $5336(2)$ & $8154(2)$ & $24(1)$ \\
\hline$C(25)$ & $6656(3)$ & $5265(2)$ & $7822(2)$ & $26(1)$ \\
\hline$C(26)$ & $6283(3)$ & $4595(2)$ & $7445(2)$ & $22(1)$ \\
\hline$C(27)$ & $6315(2)$ & $2926(2)$ & $8752(2)$ & $16(1)$ \\
\hline$C(28)$ & 7004(3) & $3459(2)$ & $9050(2)$ & $22(1)$ \\
\hline$C(29)$ & 6886(3) & 4099(2) & $9430(2)$ & $28(1)$ \\
\hline $\mathrm{C}(30)$ & 6106(3) & $4207(2)$ & $9526(2)$ & $29(1)$ \\
\hline$C(31)$ & $5425(3)$ & $3691(2)$ & $9241(2)$ & $26(1)$ \\
\hline$C(32)$ & $5528(2)$ & $3054(2)$ & $8852(2)$ & $20(1)$ \\
\hline$C(33)$ & 7363(2) & $1567(2)$ & 8954(2) & $15(1)$ \\
\hline$C(34)$ & $7390(2)$ & $1519(2)$ & $9677(2)$ & 19(1) \\
\hline$C(35)$ & $8016(3)$ & $1065(2)$ & 10198(2) & $24(1)$ \\
\hline
\end{tabular}




\begin{tabular}{lrrrr}
$\mathrm{C}(36)$ & $8626(3)$ & $665(2)$ & $10004(2)$ & $27(1)$ \\
$\mathrm{C}(37)$ & $8613(3)$ & $713(2)$ & $9295(2)$ & $26(1)$ \\
$\mathrm{C}(38)$ & $7981(2)$ & $1157(2)$ & $8772(2)$ & $22(1)$ \\
$\mathrm{C}(39)$ & $6308(2)$ & $-23(2)$ & $8558(2)$ & $19(1)$ \\
$\mathrm{C}(40)$ & $6717(2)$ & $-548(2)$ & $9121(2)$ & $22(1)$ \\
$\mathrm{C}(41)$ & $6546(2)$ & $-497(2)$ & $9763(2)$ & $22(1)$ \\
$\mathrm{C}(42)$ & $5964(2)$ & $63(2)$ & $9812(2)$ & $20(1)$ \\
$\mathrm{C}(43)$ & $5555(2)$ & $559(2)$ & $9224(2)$ & $17(1)$ \\
$\mathrm{C}(44)$ & $4924(2)$ & $1156(2)$ & $9259(2)$ & $19(1)$ \\
$\mathrm{C}(45)$ & $5112(3)$ & $1601(2)$ & $9897(2)$ & $24(1)$ \\
$\mathrm{C}(46)$ & $4537(3)$ & $2167(2)$ & $9935(2)$ & $28(1)$ \\
$\mathrm{C}(47)$ & $3764(3)$ & $2297(2)$ & $9341(2)$ & $29(1)$ \\
$\mathrm{C}(48)$ & $3549(3)$ & $1861(2)$ & $8707(2)$ & $24(1)$ \\
$\mathrm{C}(49)$ & $4122(2)$ & $1288(2)$ & $8664(2)$ & $20(1)$ \\
$\mathrm{C}(50)$ & $3261(3)$ & $-466(2)$ & $6256(2)$ & $30(1)$ \\
& & & & \\
$\mathrm{Cl}(1)$ & $5361(1)$ & $7363(1)$ & $6968(1)$ & $69(1)$ \\
$\mathrm{Cl}(2)$ & $5166(1)$ & $6815(1)$ & $8296(1)$ & $51(1)$ \\
$\mathrm{C}(51)$ & $5350(3)$ & $7629(2)$ & $7826(2)$ & $49(1)$ \\
$\mathrm{Cl}(3)$ & & & & \\
$\mathrm{Cl}(4)$ & $916(1)$ & $-46(1)$ & $6878(1)$ & $53(1)$ \\
$\mathrm{C}(52)$ & $1633(1)$ & $513(1)$ & $8389(1)$ & $67(1)$ \\
& $1777(3)$ & $476(2)$ & $7541(2)$ & $45(1)$ \\
\hline
\end{tabular}


Table 8. Bond lengths $[\AA]$ and angles $\left[{ }^{\circ}\right]$ for 5 (CCDC 172948).

\begin{tabular}{|c|c|c|c|}
\hline $\operatorname{Pd}(1)-\mathrm{N}(1)$ & $2.139(3)$ & $C(15)-C(16)$ & $1.398(5)$ \\
\hline $\operatorname{Pd}(1)-O(1)$ & $2.195(2)$ & $C(16)-C(17)$ & $1.397(5)$ \\
\hline $\mathrm{Pd}(1)-\mathrm{P}(2)$ & $2.2503(9)$ & $\mathrm{C}(16)-\mathrm{H}(16)$ & $0.91(3)$ \\
\hline $\operatorname{Pd}(1)-\mathrm{P}(1)$ & $2.2718(9)$ & $\mathrm{C}(17)-\mathrm{C}(18)$ & $1.383(5)$ \\
\hline $\mathrm{S}(1)-\mathrm{O}(3)$ & $1.436(2)$ & $\mathrm{C}(17)-\mathrm{H}(17)$ & $0.86(3)$ \\
\hline $\mathrm{S}(1)-\mathrm{O}(2)$ & $1.436(2)$ & $\mathrm{C}(18)-\mathrm{C}(19)$ & $1.380(5)$ \\
\hline $\mathrm{S}(1)-\mathrm{O}(1)$ & $1.474(2)$ & $\mathrm{C}(18)-\mathrm{H}(18)$ & $0.93(3)$ \\
\hline$S(1)-C(50)$ & $1.828(4)$ & C(19)-C(20) & $1.399(5)$ \\
\hline $\mathrm{P}(1)-\mathrm{C}(1)$ & $1.790(4)$ & $\mathrm{C}(19)-\mathrm{H}(19)$ & $0.84(3)$ \\
\hline $\mathrm{P}(1)-\mathrm{C}(9)$ & $1.811(3)$ & $\mathrm{C}(20)-\mathrm{H}(20)$ & $0.94(3)$ \\
\hline$P(1)-C(3)$ & $1.824(3)$ & $C(21)-C(22)$ & $1.410(5)$ \\
\hline$P(2)-C(2)$ & $1.789(4)$ & $C(21)-C(26)$ & $1.405(5)$ \\
\hline $\mathrm{P}(2)-\mathrm{C}(27)$ & $1.812(3)$ & $C(22)-C(23)$ & $1.383(5)$ \\
\hline $\mathrm{P}(2)-\mathrm{C}(33)$ & $1.826(3)$ & $\mathrm{C}(22)-\mathrm{H}(22)$ & $0.94(3)$ \\
\hline $\mathrm{F}(1)-\mathrm{C}(50)$ & $1.343(4)$ & $\mathrm{C}(23)-\mathrm{C}(24)$ & $1.379(5)$ \\
\hline $\mathrm{F}(2)-\mathrm{C}(50)$ & $1.325(4)$ & $\mathrm{C}(23)-\mathrm{H}(23)$ & $0.81(3)$ \\
\hline $\mathrm{F}(3)-\mathrm{C}(50)$ & $1.335(4)$ & $C(24)-C(25)$ & $1.388(5)$ \\
\hline $\mathrm{N}(1)-\mathrm{C}(43)$ & $1.355(4)$ & $\mathrm{C}(24)-\mathrm{H}(24)$ & $0.99(3)$ \\
\hline $\mathrm{N}(1)-\mathrm{C}(39)$ & $1.353(4)$ & $C(25)-C(26)$ & $1.392(5)$ \\
\hline $\mathrm{B}(1)-\mathrm{C}(15)$ & $1.636(5)$ & $\mathrm{C}(25)-\mathrm{H}(25)$ & $0.97(3)$ \\
\hline $\mathrm{B}(1)-\mathrm{C}(21)$ & $1.640(5)$ & $\mathrm{C}(26)-\mathrm{H}(26)$ & $0.87(3)$ \\
\hline B(1)-C(1) & $1.672(5)$ & $\mathrm{C}(27)-\mathrm{C}(32)$ & $1.393(5)$ \\
\hline B(1)-C(2) & $1.685(5)$ & $\mathrm{C}(27)-\mathrm{C}(28)$ & $1.406(5)$ \\
\hline $\mathrm{C}(1)-\mathrm{H}(1 \mathrm{~A})$ & $0.89(3)$ & $C(28)-C(29)$ & $1.393(5)$ \\
\hline $\mathrm{C}(1)-\mathrm{H}(1 \mathrm{~B})$ & $0.94(3)$ & $\mathrm{C}(28)-\mathrm{H}(28)$ & $0.95(3)$ \\
\hline $\mathrm{C}(2)-\mathrm{H}(2 \mathrm{~A})$ & $0.95(3)$ & $C(29)-C(30)$ & $1.370(5)$ \\
\hline $\mathrm{C}(2)-\mathrm{H}(2 \mathrm{~B})$ & $0.92(3)$ & $\mathrm{C}(29)-\mathrm{H}(29)$ & $0.83(3)$ \\
\hline C(3)-C(8) & $1.396(5)$ & $C(30)-C(31)$ & $1.375(5)$ \\
\hline$C(3)-C(4)$ & $1.404(5)$ & $\mathrm{C}(30)-\mathrm{H}(30)$ & $0.86(3)$ \\
\hline$C(4)-C(5)$ & $1.378(5)$ & $\mathrm{C}(31)-\mathrm{C}(32)$ & $1.390(5)$ \\
\hline $\mathrm{C}(4)-\mathrm{H}(4)$ & $0.83(3)$ & $\mathrm{C}(31)-\mathrm{H}(31)$ & $0.90(3)$ \\
\hline$C(5)-C(6)$ & $1.383(5)$ & $\mathrm{C}(32)-\mathrm{H}(32)$ & $0.83(3)$ \\
\hline $\mathrm{C}(5)-\mathrm{H}(5)$ & $0.86(3)$ & $\mathrm{C}(33)-\mathrm{C}(38)$ & $1.390(5)$ \\
\hline$C(6)-C(7)$ & $1.384(5)$ & $\mathrm{C}(33)-\mathrm{C}(34)$ & $1.399(5)$ \\
\hline $\mathrm{C}(6)-\mathrm{H}(6)$ & $0.92(3)$ & $C(34)-C(35)$ & $1.387(5)$ \\
\hline$C(7)-C(8)$ & $1.391(5)$ & $\mathrm{C}(34)-\mathrm{H}(34)$ & $0.91(3)$ \\
\hline $\mathrm{C}(7)-\mathrm{H}(7)$ & $0.88(3)$ & $C(35)-C(36)$ & $1.381(5)$ \\
\hline $\mathrm{C}(8)-\mathrm{H}(8)$ & $0.91(3)$ & $\mathrm{C}(35)-\mathrm{H}(35)$ & $0.79(3)$ \\
\hline $\mathrm{C}(9)-\mathrm{C}(10)$ & $1.389(4)$ & $C(36)-C(37)$ & $1.381(5)$ \\
\hline $\mathrm{C}(9)-\mathrm{C}(14)$ & $1.395(4)$ & $\mathrm{C}(36)-\mathrm{H}(36)$ & $0.84(3)$ \\
\hline $\mathrm{C}(10)-\mathrm{C}(11)$ & $1.394(5)$ & $\mathrm{C}(37)-\mathrm{C}(38)$ & $1.383(5)$ \\
\hline $\mathrm{C}(10)-\mathrm{H}(10)$ & $0.88(3)$ & $\mathrm{C}(37)-\mathrm{H}(37)$ & $0.84(3)$ \\
\hline $\mathrm{C}(11)-\mathrm{C}(12)$ & $1.384(5)$ & $\mathrm{C}(38)-\mathrm{H}(38)$ & $0.86(3)$ \\
\hline $\mathrm{C}(11)-\mathrm{H}(11)$ & $0.93(3)$ & $C(39)-C(40)$ & $1.392(5)$ \\
\hline$C(12)-C(13)$ & $1.390(5)$ & $\mathrm{C}(39)-\mathrm{H}(39)$ & $0.91(3)$ \\
\hline $\mathrm{C}(12)-\mathrm{H}(12)$ & $0.95(3)$ & $\mathrm{C}(40)-\mathrm{C}(41)$ & $1.389(5)$ \\
\hline $\mathrm{C}(13)-\mathrm{C}(14)$ & $1.385(5)$ & $\mathrm{C}(40)-\mathrm{H}(40)$ & $0.93(3)$ \\
\hline $\mathrm{C}(13)-\mathrm{H}(13)$ & $0.89(3)$ & $\mathrm{C}(41)-\mathrm{C}(42)$ & $1.390(5)$ \\
\hline $\mathrm{C}(14)-\mathrm{H}(14)$ & $0.92(3)$ & $\mathrm{C}(41)-\mathrm{H}(41)$ & $0.93(3)$ \\
\hline $\mathrm{C}(15)-\mathrm{C}(20)$ & $1.401(4)$ & $C(42)-C(43)$ & $1.391(4)$ \\
\hline
\end{tabular}




\begin{tabular}{|c|c|c|c|}
\hline $\mathrm{C}(42)-\mathrm{H}(42)$ & $0.95(3)$ & $\mathrm{C}(21)-\mathrm{B}(1)-\mathrm{C}(2)$ & $109.0(3)$ \\
\hline $\mathrm{C}(43)-\mathrm{C}(44)$ & $1.486(4)$ & $\mathrm{C}(1)-\mathrm{B}(1)-\mathrm{C}(2)$ & $109.5(3)$ \\
\hline $\mathrm{C}(44)-\mathrm{C}(45)$ & $1.399(5)$ & $\mathrm{B}(1)-\mathrm{C}(1)-\mathrm{P}(1)$ & $118.2(2)$ \\
\hline $\mathrm{C}(44)-\mathrm{C}(49)$ & $1.404(5)$ & $\mathrm{B}(1)-\mathrm{C}(1)-\mathrm{H}(1 \mathrm{~A})$ & $111.7(18)$ \\
\hline $\mathrm{C}(45)-\mathrm{C}(46)$ & $1.385(5)$ & $\mathrm{P}(1)-\mathrm{C}(1)-\mathrm{H}(1 \mathrm{~A})$ & $104.3(18)$ \\
\hline $\mathrm{C}(45)-\mathrm{H}(45)$ & $0.89(3)$ & $\mathrm{B}(1)-\mathrm{C}(1)-\mathrm{H}(1 \mathrm{~B})$ & $111.9(17)$ \\
\hline $\mathrm{C}(46)-\mathrm{C}(47)$ & $1.375(6)$ & $\mathrm{P}(1)-\mathrm{C}(1)-\mathrm{H}(1 \mathrm{~B})$ & $102.6(16)$ \\
\hline $\mathrm{C}(46)-\mathrm{H}(46)$ & $0.91(3)$ & $\mathrm{H}(1 \mathrm{~A})-\mathrm{C}(1)-\mathrm{H}(1 \mathrm{~B})$ & $107(2)$ \\
\hline $\mathrm{C}(47)-\mathrm{C}(48)$ & $1.381(5)$ & $\mathrm{B}(1)-\mathrm{C}(2)-\mathrm{P}(2)$ & $118.1(2)$ \\
\hline $\mathrm{C}(47)-\mathrm{H}(47)$ & $0.77(3)$ & $\mathrm{B}(1)-\mathrm{C}(2)-\mathrm{H}(2 \mathrm{~A})$ & $112.4(18)$ \\
\hline $\mathrm{C}(48)-\mathrm{C}(49)$ & $1.393(5)$ & $\mathrm{P}(2)-\mathrm{C}(2)-\mathrm{H}(2 \mathrm{~A})$ & $102.8(18)$ \\
\hline $\mathrm{C}(48)-\mathrm{H}(48)$ & $1.02(3)$ & $\mathrm{B}(1)-\mathrm{C}(2)-\mathrm{H}(2 \mathrm{~B})$ & $110.0(19)$ \\
\hline $\mathrm{C}(49)-\mathrm{H}(49)$ & $0.98(3)$ & $\mathrm{P}(2)-\mathrm{C}(2)-\mathrm{H}(2 \mathrm{~B})$ & $108.7(19)$ \\
\hline $\mathrm{Cl}(1)-\mathrm{C}(51)$ & $1.746(4)$ & $\mathrm{H}(2 \mathrm{~A})-\mathrm{C}(2)-\mathrm{H}(2 \mathrm{~B})$ & $104(3)$ \\
\hline $\mathrm{Cl}(2)-\mathrm{C}(51)$ & $1.776(4)$ & $\mathrm{C}(8)-\mathrm{C}(3)-\mathrm{C}(4)$ & $117.8(3)$ \\
\hline $\mathrm{C}(51)-\mathrm{H}(51 \mathrm{~A})$ & 0.9900 & $\mathrm{C}(8)-\mathrm{C}(3)-\mathrm{P}(1)$ & $122.0(3)$ \\
\hline $\mathrm{C}(51)-\mathrm{H}(51 \mathrm{~B})$ & 0.9900 & $\mathrm{C}(4)-\mathrm{C}(3)-\mathrm{P}(1)$ & $120.2(3)$ \\
\hline $\mathrm{Cl}(3)-\mathrm{C}(52)$ & $1.764(4)$ & $\mathrm{C}(5)-\mathrm{C}(4)-\mathrm{C}(3)$ & $121.4(4)$ \\
\hline $\mathrm{Cl}(4)-\mathrm{C}(52)$ & $1.761(4)$ & $\mathrm{C}(5)-\mathrm{C}(4)-\mathrm{H}(4)$ & $120(2)$ \\
\hline $\mathrm{C}(52)-\mathrm{H}(52 \mathrm{~A})$ & 0.9900 & $\mathrm{C}(3)-\mathrm{C}(4)-\mathrm{H}(4)$ & $119(2)$ \\
\hline \multirow[t]{2}{*}{$\mathrm{C}(52)-\mathrm{H}(52 \mathrm{~B})$} & 0.9900 & $\mathrm{C}(4)-\mathrm{C}(5)-\mathrm{C}(6)$ & $120.2(4)$ \\
\hline & & $\mathrm{C}(4)-\mathrm{C}(5)-\mathrm{H}(5)$ & $120(2)$ \\
\hline $\mathrm{N}(1)-\mathrm{Pd}(1)-\mathrm{O}(1)$ & $89.85(9)$ & $\mathrm{C}(6)-\mathrm{C}(5)-\mathrm{H}(5)$ & $120(2)$ \\
\hline N(1)-Pd(1)-P(2) & 93.87(7) & $C(7)-C(6)-C(5)$ & $119.7(4)$ \\
\hline $\mathrm{O}(1)-\mathrm{Pd}(1)-\mathrm{P}(2)$ & $171.45(6)$ & $\mathrm{C}(7)-\mathrm{C}(6)-\mathrm{H}(6)$ & $122(2)$ \\
\hline $\mathrm{N}(1)-\operatorname{Pd}(1)-\mathrm{P}(1)$ & $177.49(7)$ & $\mathrm{C}(5)-\mathrm{C}(6)-\mathrm{H}(6)$ & $118(2)$ \\
\hline $\mathrm{O}(1)-\mathrm{Pd}(1)-\mathrm{P}(1)$ & $88.00(6)$ & $\mathrm{C}(6)-\mathrm{C}(7)-\mathrm{C}(8)$ & $120.4(4)$ \\
\hline $\mathrm{P}(2)-\mathrm{Pd}(1)-\mathrm{P}(1)$ & $88.43(3)$ & $\mathrm{C}(6)-\mathrm{C}(7)-\mathrm{H}(7)$ & $118(2)$ \\
\hline $\mathrm{O}(3)-\mathrm{S}(1)-\mathrm{O}(2)$ & $117.33(15)$ & $\mathrm{C}(8)-\mathrm{C}(7)-\mathrm{H}(7)$ & $122(2)$ \\
\hline $\mathrm{O}(3)-\mathrm{S}(1)-\mathrm{O}(1)$ & $113.40(15)$ & $\mathrm{C}(3)-\mathrm{C}(8)-\mathrm{C}(7)$ & $120.7(4)$ \\
\hline $\mathrm{O}(2)-\mathrm{S}(1)-\mathrm{O}(1)$ & $114.16(14)$ & $\mathrm{C}(3)-\mathrm{C}(8)-\mathrm{H}(8)$ & 117.1(19) \\
\hline $\mathrm{O}(3)-\mathrm{S}(1)-\mathrm{C}(50)$ & $104.44(17)$ & $\mathrm{C}(7)-\mathrm{C}(8)-\mathrm{H}(8)$ & $121.7(19)$ \\
\hline $\mathrm{O}(2)-\mathrm{S}(1)-\mathrm{C}(50)$ & $103.84(16)$ & $\mathrm{C}(10)-\mathrm{C}(9)-\mathrm{C}(14)$ & $119.1(3)$ \\
\hline $\mathrm{O}(1)-\mathrm{S}(1)-\mathrm{C}(50)$ & $101.15(15)$ & $\mathrm{C}(10)-\mathrm{C}(9)-\mathrm{P}(1)$ & $120.1(3)$ \\
\hline $\mathrm{C}(1)-\mathrm{P}(1)-\mathrm{C}(9)$ & $105.88(16)$ & C(14)-C(9)-P(1) & $120.3(3)$ \\
\hline $\mathrm{C}(1)-\mathrm{P}(1)-\mathrm{C}(3)$ & $107.37(16)$ & $\mathrm{C}(9)-\mathrm{C}(10)-\mathrm{C}(11)$ & $120.3(3)$ \\
\hline C(9)-P(1)-C(3) & $108.08(15)$ & $\mathrm{C}(9)-\mathrm{C}(10)-\mathrm{H}(10)$ & $117(2)$ \\
\hline $\mathrm{C}(1)-\mathrm{P}(1)-\mathrm{Pd}(1)$ & $119.44(12)$ & $\mathrm{C}(11)-\mathrm{C}(10)-\mathrm{H}(10)$ & 123(2) \\
\hline $\mathrm{C}(9)-\mathrm{P}(1)-\mathrm{Pd}(1)$ & $110.25(11)$ & $C(12)-C(11)-C(10)$ & $120.3(4)$ \\
\hline C(3)-P(1)-Pd(1) & $105.38(10)$ & $\mathrm{C}(12)-\mathrm{C}(11)-\mathrm{H}(11)$ & $119(2)$ \\
\hline C(2)-P(2)-C(27) & $109.88(16)$ & $\mathrm{C}(10)-\mathrm{C}(11)-\mathrm{H}(11)$ & $121(2)$ \\
\hline C(2)-P(2)-C(33) & $107.81(16)$ & $\mathrm{C}(11)-\mathrm{C}(12)-\mathrm{C}(13)$ & $119.6(3)$ \\
\hline $\mathrm{C}(27)-\mathrm{P}(2)-\mathrm{C}(33)$ & $103.63(15)$ & $\mathrm{C}(11)-\mathrm{C}(12)-\mathrm{H}(12)$ & 123.3(19) \\
\hline$C(2)-P(2)-P d(1)$ & $110.91(13)$ & $\mathrm{C}(13)-\mathrm{C}(12)-\mathrm{H}(12)$ & 117.1(18) \\
\hline $\mathrm{C}(27)-\mathrm{P}(2)-\mathrm{Pd}(1)$ & $114.64(12)$ & $\mathrm{C}(14)-\mathrm{C}(13)-\mathrm{C}(12)$ & $120.2(4)$ \\
\hline $\mathrm{C}(33)-\mathrm{P}(2)-\mathrm{Pd}(1)$ & $109.53(10)$ & $\mathrm{C}(14)-\mathrm{C}(13)-\mathrm{H}(13)$ & $118(2)$ \\
\hline $\mathrm{C}(43)-\mathrm{N}(1)-\mathrm{C}(39)$ & $119.0(3)$ & $\mathrm{C}(12)-\mathrm{C}(13)-\mathrm{H}(13)$ & $121(2)$ \\
\hline $\mathrm{C}(43)-\mathrm{N}(1)-\mathrm{Pd}(1)$ & $123.6(2)$ & $\mathrm{C}(13)-\mathrm{C}(14)-\mathrm{C}(9)$ & $120.5(3)$ \\
\hline $\mathrm{C}(39)-\mathrm{N}(1)-\mathrm{Pd}(1)$ & $117.0(2)$ & $\mathrm{C}(13)-\mathrm{C}(14)-\mathrm{H}(14)$ & 119.1(17) \\
\hline $\mathrm{S}(1)-\mathrm{O}(1)-\mathrm{Pd}(1)$ & $128.63(13)$ & $\mathrm{C}(9)-\mathrm{C}(14)-\mathrm{H}(14)$ & $120.3(17)$ \\
\hline $\mathrm{C}(15)-\mathrm{B}(1)-\mathrm{C}(21)$ & $108.9(3)$ & $C(20)-C(15)-C(16)$ & $114.2(3)$ \\
\hline $\mathrm{C}(15)-\mathrm{B}(1)-\mathrm{C}(1)$ & $112.2(3)$ & $\mathrm{C}(20)-\mathrm{C}(15)-\mathrm{B}(1)$ & $121.2(3)$ \\
\hline $\mathrm{C}(21)-\mathrm{B}(1)-\mathrm{C}(1)$ & $107.7(3)$ & $\mathrm{C}(16)-\mathrm{C}(15)-\mathrm{B}(1)$ & $124.5(3)$ \\
\hline $\mathrm{C}(15)-\mathrm{B}(1)-\mathrm{C}(2)$ & $109.5(3)$ & $C(15)-C(16)-C(17)$ & $123.4(4)$ \\
\hline
\end{tabular}




\begin{tabular}{|c|c|c|c|}
\hline $\mathrm{C}(15)-\mathrm{C}(16)-\mathrm{H}(16)$ & $118(2)$ & $\mathrm{C}(35)-\mathrm{C}(34)-\mathrm{H}(34)$ & $118.9(19)$ \\
\hline $\mathrm{C}(17)-\mathrm{C}(16)-\mathrm{H}(16)$ & $118(2)$ & $\mathrm{C}(33)-\mathrm{C}(34)-\mathrm{H}(34)$ & $120.4(19)$ \\
\hline$C(18)-C(17)-C(16)$ & $120.4(4)$ & $C(36)-C(35)-C(34)$ & $119.7(4)$ \\
\hline $\mathrm{C}(18)-\mathrm{C}(17)-\mathrm{H}(17)$ & $120(2)$ & $\mathrm{C}(36)-\mathrm{C}(35)-\mathrm{H}(35)$ & $125(3)$ \\
\hline $\mathrm{C}(16)-\mathrm{C}(17)-\mathrm{H}(17)$ & $120(2)$ & $\mathrm{C}(34)-\mathrm{C}(35)-\mathrm{H}(35)$ & $115(3)$ \\
\hline $\mathrm{C}(19)-\mathrm{C}(18)-\mathrm{C}(17)$ & $118.2(4)$ & $C(35)-C(36)-C(37)$ & $120.3(4)$ \\
\hline $\mathrm{C}(19)-\mathrm{C}(18)-\mathrm{H}(18)$ & $119(2)$ & $\mathrm{C}(35)-\mathrm{C}(36)-\mathrm{H}(36)$ & $115(2)$ \\
\hline $\mathrm{C}(17)-\mathrm{C}(18)-\mathrm{H}(18)$ & $123(2)$ & $\mathrm{C}(37)-\mathrm{C}(36)-\mathrm{H}(36)$ & $125(2)$ \\
\hline $\mathrm{C}(18)-\mathrm{C}(19)-\mathrm{C}(20)$ & $120.5(4)$ & $\mathrm{C}(38)-\mathrm{C}(37)-\mathrm{C}(36)$ & $120.0(4)$ \\
\hline $\mathrm{C}(18)-\mathrm{C}(19)-\mathrm{H}(19)$ & $119(2)$ & $\mathrm{C}(38)-\mathrm{C}(37)-\mathrm{H}(37)$ & $116(2)$ \\
\hline $\mathrm{C}(20)-\mathrm{C}(19)-\mathrm{H}(19)$ & $120(2)$ & $\mathrm{C}(36)-\mathrm{C}(37)-\mathrm{H}(37)$ & $124(2)$ \\
\hline$C(15)-C(20)-C(19)$ & $123.2(4)$ & $\mathrm{C}(33)-\mathrm{C}(38)-\mathrm{C}(37)$ & $120.8(4)$ \\
\hline $\mathrm{C}(15)-\mathrm{C}(20)-\mathrm{H}(20)$ & 116.2(19) & $\mathrm{C}(33)-\mathrm{C}(38)-\mathrm{H}(38)$ & $116(2)$ \\
\hline $\mathrm{C}(19)-\mathrm{C}(20)-\mathrm{H}(20)$ & $121(2)$ & $\mathrm{C}(37)-\mathrm{C}(38)-\mathrm{H}(38)$ & $123(2)$ \\
\hline $\mathrm{C}(22)-\mathrm{C}(21)-\mathrm{C}(26)$ & $114.4(3)$ & N(1)-C(39)-C(40) & $123.0(3)$ \\
\hline $\mathrm{C}(22)-\mathrm{C}(21)-\mathrm{B}(1)$ & $121.0(3)$ & N(1)-C(39)-H(39) & $119.2(18)$ \\
\hline $\mathrm{C}(26)-\mathrm{C}(21)-\mathrm{B}(1)$ & $124.6(3)$ & $\mathrm{C}(40)-\mathrm{C}(39)-\mathrm{H}(39)$ & $117.9(18)$ \\
\hline$C(23)-C(22)-C(21)$ & $122.8(4)$ & $C(39)-C(40)-C(41)$ & $118.1(4)$ \\
\hline $\mathrm{C}(23)-\mathrm{C}(22)-\mathrm{H}(22)$ & 115.1(19) & $\mathrm{C}(39)-\mathrm{C}(40)-\mathrm{H}(40)$ & $117(2)$ \\
\hline $\mathrm{C}(21)-\mathrm{C}(22)-\mathrm{H}(22)$ & $122.0(19)$ & $\mathrm{C}(41)-\mathrm{C}(40)-\mathrm{H}(40)$ & $125(2)$ \\
\hline$C(22)-C(23)-C(24)$ & $121.0(4)$ & $C(42)-C(41)-C(40)$ & $118.9(3)$ \\
\hline $\mathrm{C}(22)-\mathrm{C}(23)-\mathrm{H}(23)$ & $115(2)$ & $\mathrm{C}(42)-\mathrm{C}(41)-\mathrm{H}(41)$ & $124(2)$ \\
\hline $\mathrm{C}(24)-\mathrm{C}(23)-\mathrm{H}(23)$ & $124(2)$ & $\mathrm{C}(40)-\mathrm{C}(41)-\mathrm{H}(41)$ & $117(2)$ \\
\hline$C(25)-C(24)-C(23)$ & $118.4(4)$ & $\mathrm{C}(43)-\mathrm{C}(42)-\mathrm{C}(41)$ & $120.5(3)$ \\
\hline $\mathrm{C}(25)-\mathrm{C}(24)-\mathrm{H}(24)$ & $121.0(18)$ & $\mathrm{C}(43)-\mathrm{C}(42)-\mathrm{H}(42)$ & $119.4(17)$ \\
\hline $\mathrm{C}(23)-\mathrm{C}(24)-\mathrm{H}(24)$ & $120.6(18)$ & $\mathrm{C}(41)-\mathrm{C}(42)-\mathrm{H}(42)$ & $120.0(17)$ \\
\hline$C(26)-C(25)-C(24)$ & $120.1(4)$ & N(1)-C(43)-C(42) & $120.4(3)$ \\
\hline $\mathrm{C}(26)-\mathrm{C}(25)-\mathrm{H}(25)$ & $118(2)$ & N(1)-C(43)-C(44) & $118.0(3)$ \\
\hline $\mathrm{C}(24)-\mathrm{C}(25)-\mathrm{H}(25)$ & 121(2) & $\mathrm{C}(42)-\mathrm{C}(43)-\mathrm{C}(44)$ & $121.5(3)$ \\
\hline$C(25)-C(26)-C(21)$ & $123.2(4)$ & $C(45)-C(44)-C(49)$ & $118.0(3)$ \\
\hline$C(25)-C(26)-H(26)$ & $117(2)$ & $C(45)-C(44)-C(43)$ & $120.4(3)$ \\
\hline $\mathrm{C}(21)-\mathrm{C}(26)-\mathrm{H}(26)$ & $120(2)$ & $\mathrm{C}(49)-\mathrm{C}(44)-\mathrm{C}(43)$ & $121.6(3)$ \\
\hline$C(32)-C(27)-C(28)$ & $118.4(3)$ & $C(46)-C(45)-C(44)$ & $121.2(4)$ \\
\hline C(32)-C(27)-P(2) & $123.0(3)$ & $\mathrm{C}(46)-\mathrm{C}(45)-\mathrm{H}(45)$ & $119(2)$ \\
\hline $\mathrm{C}(28)-\mathrm{C}(27)-\mathrm{P}(2)$ & $118.6(3)$ & $\mathrm{C}(44)-\mathrm{C}(45)-\mathrm{H}(45)$ & $120(2)$ \\
\hline $\mathrm{C}(29)-\mathrm{C}(28)-\mathrm{C}(27)$ & $119.9(4)$ & $C(45)-C(46)-C(47)$ & $119.9(4)$ \\
\hline $\mathrm{C}(29)-\mathrm{C}(28)-\mathrm{H}(28)$ & $122.4(19)$ & $\mathrm{C}(45)-\mathrm{C}(46)-\mathrm{H}(46)$ & $121(2)$ \\
\hline $\mathrm{C}(27)-\mathrm{C}(28)-\mathrm{H}(28)$ & $117.7(19)$ & $\mathrm{C}(47)-\mathrm{C}(46)-\mathrm{H}(46)$ & $119(2)$ \\
\hline$C(30)-C(29)-C(28)$ & $120.5(4)$ & $C(48)-C(47)-C(46)$ & $120.6(4)$ \\
\hline $\mathrm{C}(30)-\mathrm{C}(29)-\mathrm{H}(29)$ & $120(2)$ & $\mathrm{C}(48)-\mathrm{C}(47)-\mathrm{H}(47)$ & $120(3)$ \\
\hline $\mathrm{C}(28)-\mathrm{C}(29)-\mathrm{H}(29)$ & $119(2)$ & $\mathrm{C}(46)-\mathrm{C}(47)-\mathrm{H}(47)$ & $120(3)$ \\
\hline$C(29)-C(30)-C(31)$ & $120.5(4)$ & $C(47)-C(48)-C(49)$ & $119.9(4)$ \\
\hline $\mathrm{C}(29)-\mathrm{C}(30)-\mathrm{H}(30)$ & $118(2)$ & $\mathrm{C}(47)-\mathrm{C}(48)-\mathrm{H}(48)$ & $120.8(18)$ \\
\hline $\mathrm{C}(31)-\mathrm{C}(30)-\mathrm{H}(30)$ & $120(2)$ & $\mathrm{C}(49)-\mathrm{C}(48)-\mathrm{H}(48)$ & $119.2(18)$ \\
\hline $\mathrm{C}(30)-\mathrm{C}(31)-\mathrm{C}(32)$ & $119.8(4)$ & $C(48)-C(49)-C(44)$ & $120.4(3)$ \\
\hline $\mathrm{C}(30)-\mathrm{C}(31)-\mathrm{H}(31)$ & $120(2)$ & $\mathrm{C}(48)-\mathrm{C}(49)-\mathrm{H}(49)$ & $121.3(17)$ \\
\hline $\mathrm{C}(32)-\mathrm{C}(31)-\mathrm{H}(31)$ & $120(2)$ & $\mathrm{C}(44)-\mathrm{C}(49)-\mathrm{H}(49)$ & $118.3(17)$ \\
\hline$C(27)-C(32)-C(31)$ & $120.9(4)$ & $\mathrm{F}(2)-\mathrm{C}(50)-\mathrm{F}(3)$ & 108.3(3) \\
\hline $\mathrm{C}(27)-\mathrm{C}(32)-\mathrm{H}(32)$ & $120(2)$ & $\mathrm{F}(2)-\mathrm{C}(50)-\mathrm{F}(1)$ & 107.3(3) \\
\hline $\mathrm{C}(31)-\mathrm{C}(32)-\mathrm{H}(32)$ & $119(2)$ & $\mathrm{F}(3)-\mathrm{C}(50)-\mathrm{F}(1)$ & $107.5(3)$ \\
\hline$C(38)-C(33)-C(34)$ & $118.5(3)$ & $\mathrm{F}(2)-\mathrm{C}(50)-\mathrm{S}(1)$ & $111.7(3)$ \\
\hline C(38)-C(33)-P(2) & $120.7(3)$ & $\mathrm{F}(3)-\mathrm{C}(50)-\mathrm{S}(1)$ & $110.3(3)$ \\
\hline C(34)-C(33)-P(2) & $120.6(3)$ & F(1)-C(50)-S(1) & $111.5(3)$ \\
\hline$C(35)-C(34)-C(33)$ & $120.6(4)$ & $\mathrm{Cl}(1)-\mathrm{C}(51)-\mathrm{Cl}(2)$ & $110.5(2)$ \\
\hline
\end{tabular}


$\mathrm{Cl}(1)-\mathrm{C}(51)-\mathrm{H}(51 \mathrm{~A}) \quad 109.5$

$\mathrm{Cl}(2)-\mathrm{C}(51)-\mathrm{H}(51 \mathrm{~A}) \quad 109.5$

$\mathrm{Cl}(1)-\mathrm{C}(51)-\mathrm{H}(51 \mathrm{~B}) \quad 109.5$

$\mathrm{Cl}(2)-\mathrm{C}(51)-\mathrm{H}(51 \mathrm{~B}) \quad 109.5$

$\mathrm{H}(51 \mathrm{~A})-\mathrm{C}(51)-\mathrm{H}(51 \mathrm{~B}) \quad 108.1$

$\mathrm{Cl}(4)-\mathrm{C}(52)-\mathrm{Cl}(3) \quad 110.9(2)$

$\mathrm{Cl}(4)-\mathrm{C}(52)-\mathrm{H}(52 \mathrm{~A}) \quad 109.5$

$\mathrm{Cl}(3)-\mathrm{C}(52)-\mathrm{H}(52 \mathrm{~A}) \quad 109.5$

$\mathrm{Cl}(4)-\mathrm{C}(52)-\mathrm{H}(52 \mathrm{~B}) \quad 109.5$

$\mathrm{Cl}(3)-\mathrm{C}(52)-\mathrm{H}(52 \mathrm{~B}) \quad 109.5$

H(52A)-C(52)-H(52B) $\quad 108.0$ 
Table 9. Anisotropic displacement parameters $\left(\AA^{2} \times 10^{4}\right)$ for 5 (CCDC 172948). The anisotropic displacement factor exponent takes the form: $-2 \pi^{2}\left[h^{2} a^{* 2} U 11+\ldots+2 h k a^{*} b^{*}\right.$ $\mathbf{U}^{12}$ ]

\begin{tabular}{|c|c|c|c|c|c|c|}
\hline & $\mathrm{U}^{11}$ & $\mathrm{U}^{22}$ & $\mathrm{U}^{33}$ & $\mathrm{U}^{23}$ & $\mathrm{U}^{13}$ & $\mathrm{U}^{12}$ \\
\hline $\operatorname{Pd}(1)$ & $142(1)$ & $142(1)$ & $145(1)$ & $12(1)$ & $41(1)$ & $-12(1)$ \\
\hline $\mathrm{S}(1)$ & $217(5)$ & $190(5)$ & 191(5) & $-3(4)$ & $37(4)$ & $-43(4)$ \\
\hline $\mathrm{P}(1)$ & $144(5)$ & $140(5)$ & $150(5)$ & $4(4)$ & $49(4)$ & $-5(4)$ \\
\hline $\mathrm{P}(2)$ & $145(5)$ & $166(5)$ & $153(5)$ & $10(4)$ & $50(4)$ & $-16(4)$ \\
\hline $\mathrm{F}(1)$ & 181(12) & $515(15)$ & 411(14) & $-101(12)$ & $33(11)$ & $-14(11)$ \\
\hline $\mathrm{F}(2)$ & $380(14)$ & $571(16)$ & 204(12) & $-34(11)$ & $57(11)$ & $88(12)$ \\
\hline $\mathrm{F}(3)$ & $526(16)$ & $265(14)$ & $529(16)$ & $-127(12)$ & $-65(13)$ & $-131(12)$ \\
\hline $\mathrm{N}(1)$ & $122(15)$ & $146(15)$ & 173(16) & $10(12)$ & $40(13)$ & $-29(12)$ \\
\hline $\mathrm{O}(1)$ & $180(13)$ & $148(13)$ & 207(13) & $17(11)$ & $4(11)$ & $-44(10)$ \\
\hline $\mathrm{O}(2)$ & $230(15)$ & $277(15)$ & $334(16)$ & $-49(12)$ & $33(12)$ & $47(12)$ \\
\hline $\mathrm{O}(3)$ & $378(17)$ & $333(16)$ & 271(15) & 7(12) & $130(13)$ & $-135(13)$ \\
\hline $\mathrm{B}(1)$ & $180(20)$ & $150(20)$ & $160(20)$ & $14(17)$ & 48(18) & $-7(17)$ \\
\hline$C(1)$ & 190(20) & $168(19)$ & 117(19) & $9(16)$ & $32(17)$ & $21(16)$ \\
\hline$C(2)$ & $110(20)$ & $210(20)$ & $180(20)$ & $11(16)$ & $29(17)$ & $-16(16)$ \\
\hline$C(3)$ & $148(18)$ & $141(18)$ & $142(18)$ & $48(14)$ & $48(15)$ & $19(15)$ \\
\hline$C(4)$ & $150(20)$ & $250(20)$ & $210(20)$ & $-11(17)$ & $31(17)$ & $-11(17)$ \\
\hline$C(5)$ & $270(20)$ & $300(20)$ & $230(20)$ & $3(18)$ & 117(19) & $70(19)$ \\
\hline$C(6)$ & $170(20)$ & $380(20)$ & $270(20)$ & 103(19) & 103(19) & $77(19)$ \\
\hline$C(7)$ & $150(20)$ & $360(20)$ & $280(20)$ & 2(19) & 34(19) & $-47(19)$ \\
\hline$C(8)$ & $220(20)$ & $240(20)$ & $210(20)$ & $13(17)$ & $87(18)$ & $19(17)$ \\
\hline C(9) & $110(18)$ & $165(18)$ & 177(19) & $-9(15)$ & $61(15)$ & $-23(14)$ \\
\hline$C(10)$ & $250(20)$ & $220(20)$ & $170(20)$ & $36(16)$ & $63(18)$ & $54(17)$ \\
\hline$C(11)$ & $290(20)$ & $270(20)$ & $280(20)$ & $-38(18)$ & $130(20)$ & $97(19)$ \\
\hline$C(12)$ & $250(20)$ & $300(20)$ & 170(20) & $-66(18)$ & $90(17)$ & $-4(18)$ \\
\hline$C(13)$ & $240(20)$ & $280(20)$ & $160(20)$ & $45(17)$ & $57(18)$ & $-2(18)$ \\
\hline$C(14)$ & $200(20)$ & $170(20)$ & $220(20)$ & $14(16)$ & 61(17) & $63(17)$ \\
\hline$C(15)$ & 184(19) & $140(18)$ & $190(20)$ & $-10(15)$ & $75(16)$ & $-66(15)$ \\
\hline$C(16)$ & $290(20)$ & $210(20)$ & $220(20)$ & $52(17)$ & $125(19)$ & $2(18)$ \\
\hline$C(17)$ & $390(30)$ & $250(20)$ & $350(30)$ & $-20(19)$ & $240(20)$ & $10(20)$ \\
\hline$C(18)$ & $370(30)$ & $330(20)$ & $210(20)$ & $-50(19)$ & $170(20)$ & $-90(20)$ \\
\hline$C(19)$ & $230(20)$ & $330(30)$ & $200(20)$ & $48(18)$ & 37(19) & $-81(18)$ \\
\hline$C(20)$ & $200(20)$ & $250(20)$ & $250(20)$ & $-6(17)$ & $95(18)$ & $-28(17)$ \\
\hline $\mathrm{C}(21)$ & $210(20)$ & $195(19)$ & 123(18) & $29(15)$ & $70(16)$ & $-6(16)$ \\
\hline$C(22)$ & $230(20)$ & $200(20)$ & $220(20)$ & $20(16)$ & $106(18)$ & $-8(17)$ \\
\hline$C(23)$ & $140(20)$ & $280(20)$ & $260(20)$ & 1(18) & $35(18)$ & $-68(18)$ \\
\hline$C(24)$ & $300(20)$ & $200(20)$ & $230(20)$ & $-24(17)$ & $97(19)$ & $-91(18)$ \\
\hline$C(25)$ & $330(20)$ & $150(20)$ & $320(20)$ & $-7(17)$ & $170(20)$ & $39(18)$ \\
\hline$C(26)$ & $180(20)$ & $210(20)$ & $260(20)$ & $21(17)$ & $66(18)$ & $-27(17)$ \\
\hline $\mathrm{C}(27)$ & $175(19)$ & 178(19) & $106(18)$ & $38(15)$ & $23(15)$ & $4(15)$ \\
\hline$C(28)$ & $220(20)$ & $270(20)$ & $160(20)$ & $19(16)$ & $64(17)$ & $-19(17)$ \\
\hline$C(29)$ & $350(30)$ & $250(20)$ & $210(20)$ & $-42(18)$ & $60(20)$ & $-130(20)$ \\
\hline$C(30)$ & $440(30)$ & $200(20)$ & $220(20)$ & $-9(18)$ & $130(20)$ & $20(20)$ \\
\hline $\mathrm{C}(31)$ & $310(20)$ & $250(20)$ & $250(20)$ & $21(18)$ & 127(19) & $60(20)$ \\
\hline$C(32)$ & $190(20)$ & $210(20)$ & $170(20)$ & $22(16)$ & 41(17) & $-34(17)$ \\
\hline$C(33)$ & $113(18)$ & $135(18)$ & 179(19) & $-4(14)$ & $33(15)$ & $-41(14)$ \\
\hline$C(34)$ & $170(20)$ & $170(20)$ & $220(20)$ & $-12(15)$ & 71(17) & $-23(16)$ \\
\hline$C(35)$ & $250(20)$ & $250(20)$ & $160(20)$ & $19(17)$ & $13(19)$ & $-89(17)$ \\
\hline$C(36)$ & $180(20)$ & $220(20)$ & $300(20)$ & $63(19)$ & $-41(19)$ & $-11(18)$ \\
\hline \multirow[t]{2}{*}{$C(37)$} & $210(20)$ & $220(20)$ & $360(30)$ & $38(19)$ & $110(20)$ & $17(18)$ \\
\hline & & & & S24 & & \\
\hline
\end{tabular}




\begin{tabular}{lcllrrr}
$\mathrm{C}(38)$ & $200(20)$ & $270(20)$ & $180(20)$ & $19(17)$ & $50(18)$ & $-61(16)$ \\
$\mathrm{C}(39)$ & $160(20)$ & $220(20)$ & $180(20)$ & $10(16)$ & $58(17)$ & $-22(16)$ \\
$\mathrm{C}(40)$ & $160(20)$ & $170(20)$ & $300(20)$ & $-13(17)$ & $67(18)$ & $-20(16)$ \\
$\mathrm{C}(41)$ & $160(20)$ & $220(20)$ & $240(20)$ & $76(17)$ & $9(17)$ & $-34(17)$ \\
$\mathrm{C}(42)$ & $170(20)$ & $260(20)$ & $180(20)$ & $23(17)$ & $72(17)$ & $-57(16)$ \\
$\mathrm{C}(43)$ & $130(19)$ & $168(19)$ & $195(19)$ & $6(15)$ & $45(16)$ & $-47(15)$ \\
$\mathrm{C}(44)$ & $200(20)$ & $200(20)$ & $190(20)$ & $36(15)$ & $104(17)$ & $-38(15)$ \\
$\mathrm{C}(45)$ & $250(20)$ & $300(20)$ & $170(20)$ & $30(17)$ & $84(19)$ & $-50(18)$ \\
$\mathrm{C}(46)$ & $350(30)$ & $290(20)$ & $280(20)$ & $-66(19)$ & $210(20)$ & $-52(19)$ \\
$\mathrm{C}(47)$ & $340(30)$ & $210(20)$ & $440(30)$ & $20(20)$ & $280(20)$ & $50(20)$ \\
$\mathrm{C}(48)$ & $230(20)$ & $240(20)$ & $300(20)$ & $55(18)$ & $150(20)$ & $-15(18)$ \\
$\mathrm{C}(49)$ & $220(20)$ & $200(20)$ & $210(20)$ & $-5(17)$ & $119(17)$ & $-36(17)$ \\
$\mathrm{C}(50)$ & $280(20)$ & $250(20)$ & $320(20)$ & $-69(19)$ & $70(20)$ & $-19(19)$ \\
$\mathrm{Cl}(1)$ & $1052(12)$ & $681(9)$ & $459(8)$ & $-80(7)$ & $437(8)$ & $-262(8)$ \\
$\mathrm{Cl}(2)$ & $734(9)$ & $424(7)$ & $455(7)$ & $34(6)$ & $312(7)$ & $95(6)$ \\
$\mathrm{C}(51)$ & $700(30)$ & $330(20)$ & $490(30)$ & $40(20)$ & $300(30)$ & $-40(20)$ \\
$\mathrm{Cl}(3)$ & $503(8)$ & $511(7)$ & $487(7)$ & $-128(6)$ & $90(6)$ & $-92(6)$ \\
$\mathrm{Cl}(4)$ & $754(10)$ & $829(10)$ & $367(7)$ & $-23(7)$ & $156(7)$ & $-216(8)$ \\
$\mathrm{C}(52)$ & $470(30)$ & $510(30)$ & $310(30)$ & $-100(20)$ & $80(20)$ & $-110(20)$ \\
& & & & & \\
\hline
\end{tabular}


Table 10. Hydrogen coordinates $\left(x 1^{4}\right)$ and isotropic displacement parameters $\left(\AA^{2} \times 10^{3}\right)$ for 5 (CCDC 172948).

\begin{tabular}{|c|c|c|c|c|}
\hline & $\mathrm{x}$ & $\mathrm{y}$ & $\mathrm{z}$ & $\mathrm{U}_{\text {iso }}$ \\
\hline $\mathrm{H}(1 \mathrm{~A})$ & 4971(18) & $3384(15)$ & $6477(15)$ & $2(8)$ \\
\hline $\mathrm{H}(1 \mathrm{~B})$ & $5210(18)$ & $3355(15)$ & $7285(16)$ & $2(8)$ \\
\hline $\mathrm{H}(2 \mathrm{~A})$ & $6910(20)$ & 1963(17) & $7315(16)$ & $15(9)$ \\
\hline $\mathrm{H}(2 \mathrm{~B})$ & $7500(20)$ & $2546(17)$ & 7810(17) & $17(9)$ \\
\hline $\mathrm{H}(4)$ & $3850(18)$ & $3277(16)$ & $7365(15)$ & $0(8)$ \\
\hline $\mathrm{H}(5)$ & 2482(19) & $3530(17)$ & $7272(16)$ & 12(9) \\
\hline $\mathrm{H}(6)$ & 1330(20) & 2811(18) & $6550(18)$ & $26(10)$ \\
\hline $\mathrm{H}(7)$ & $1630(20)$ & 1808(19) & 5923(18) & $24(10)$ \\
\hline $\mathrm{H}(8)$ & 3051(19) & 1595(16) & $5963(16)$ & $9(9)$ \\
\hline $\mathrm{H}(10)$ & $5560(20)$ & 959(17) & $6395(17)$ & $14(9)$ \\
\hline $\mathrm{H}(11)$ & $5720(20)$ & 493(18) & $5323(17)$ & $23(10)$ \\
\hline $\mathrm{H}(12)$ & 4961(19) & 1137(16) & $4200(17)$ & $15(9)$ \\
\hline $\mathrm{H}(13)$ & $4120(20)$ & $2178(18)$ & $4217(17)$ & $20(10)$ \\
\hline $\mathrm{H}(14)$ & 3990(18) & 2613(15) & $5259(14)$ & $0(8)$ \\
\hline $\mathrm{H}(16)$ & $7470(20)$ & $2277(17)$ & $6487(17)$ & 13(9) \\
\hline $\mathrm{H}(17)$ & $7630(20)$ & $2203(18)$ & $5387(18)$ & $20(10)$ \\
\hline $\mathrm{H}(18)$ & $6800(20)$ & 2941(17) & $4384(17)$ & $19(9)$ \\
\hline $\mathrm{H}(19)$ & $5790(20)$ & $3702(19)$ & $4559(19)$ & $27(11)$ \\
\hline $\mathrm{H}(20)$ & $5630(20)$ & $3852(17)$ & $5669(17)$ & $19(10)$ \\
\hline $\mathrm{H}(22)$ & $8110(20)$ & 3701(18) & $7685(16)$ & $18(9)$ \\
\hline $\mathrm{H}(23)$ & $8620(20)$ & 4748(17) & $8251(16)$ & $9(9)$ \\
\hline $\mathrm{H}(24)$ & 7840(20) & $5795(17)$ & $8447(16)$ & $15(9)$ \\
\hline $\mathrm{H}(25)$ & $6260(20)$ & $5658(18)$ & 7878(17) & $25(10)$ \\
\hline $\mathrm{H}(26)$ & $5708(19)$ & 4579(16) & 7231(16) & $6(8)$ \\
\hline $\mathrm{H}(28)$ & $7530(20)$ & $3374(17)$ & $8966(17)$ & $20(10)$ \\
\hline $\mathrm{H}(29)$ & $7290(20)$ & 4421(18) & $9587(17)$ & $15(10)$ \\
\hline $\mathrm{H}(30)$ & $6020(20)$ & 4636(19) & $9707(18)$ & $27(11)$ \\
\hline $\mathrm{H}(31)$ & $4900(20)$ & 3781(18) & 9284(17) & $18(10)$ \\
\hline $\mathrm{H}(32)$ & $5120(20)$ & 2736(17) & $8694(16)$ & 11(9) \\
\hline $\mathrm{H}(34)$ & $6980(20)$ & 1769(16) & $9808(16)$ & 11(9) \\
\hline $\mathrm{H}(35)$ & $8000(20)$ & $1065(18)$ & $10597(18)$ & $16(11)$ \\
\hline $\mathrm{H}(36)$ & $8970(20)$ & 391(18) & 10341(18) & $21(10)$ \\
\hline $\mathrm{H}(37)$ & $8960(20)$ & $467(17)$ & $9147(16)$ & 11(9) \\
\hline $\mathrm{H}(38)$ & 7933(19) & 1182(16) & $8319(16)$ & $9(9)$ \\
\hline $\mathrm{H}(39)$ & 6433(19) & $-50(16)$ & $8143(15)$ & $6(8)$ \\
\hline $\mathrm{H}(40)$ & $7060(20)$ & $-926(18)$ & $9026(17)$ & $21(10)$ \\
\hline $\mathrm{H}(41)$ & $6820(20)$ & $-849(18)$ & 10134(17) & $23(10)$ \\
\hline $\mathrm{H}(42)$ & $5844(18)$ & 109(15) & $10248(15)$ & $5(8)$ \\
\hline $\mathrm{H}(45)$ & $5610(20)$ & $1519(17)$ & $10286(17)$ & $15(9)$ \\
\hline $\mathrm{H}(46)$ & $4650(20)$ & 2446(19) & 10357(19) & $32(11)$ \\
\hline $\mathrm{H}(47)$ & $3440(20)$ & 2612(18) & $9364(17)$ & $12(10)$ \\
\hline $\mathrm{H}(48)$ & $2990(20)$ & 1971(17) & $8258(17)$ & $18(9)$ \\
\hline $\mathrm{H}(49)$ & $3977(18)$ & $964(15)$ & $8226(15)$ & $4(8)$ \\
\hline $\mathrm{H}(51 \mathrm{~A})$ & 4878 & 8012 & 7755 & 73 \\
\hline $\mathrm{H}(51 \mathrm{~B})$ & 5922 & 7868 & 8128 & 73 \\
\hline $\mathrm{H}(52 \mathrm{~A})$ & 2350 & 230 & 7615 & 68 \\
\hline $\mathrm{H}(52 \mathrm{~B})$ & 1789 & 1005 & 7358 & 68 \\
\hline
\end{tabular}


Figure 3. Labeled drawing of 8 (CCDC 186799), with 50\% ellipsoids. Hydrogen atoms have been omitted for clarity.

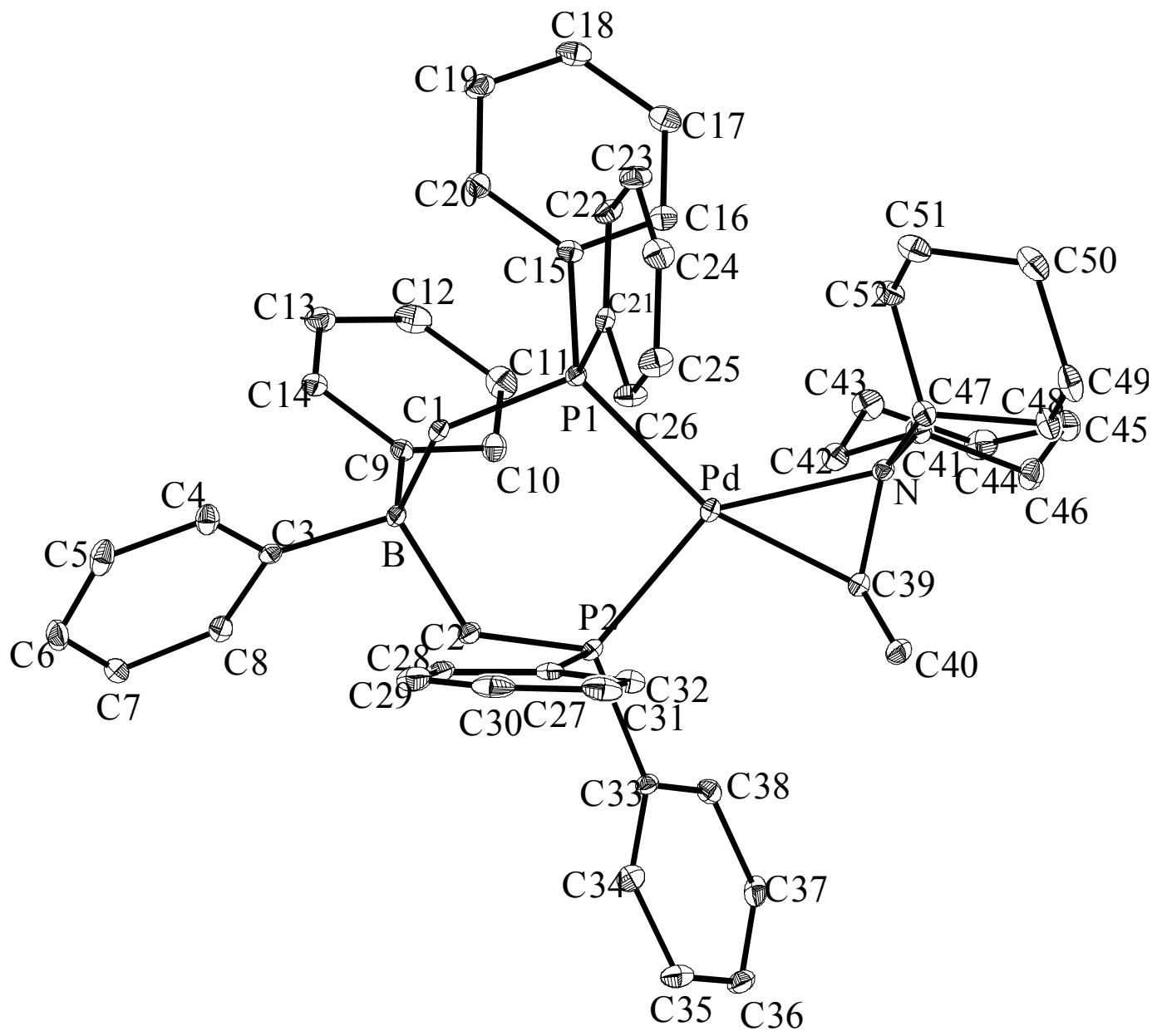


Table 11. Crystal Data and Structure Analysis Details for 8 (CCDC 186799).

Empirical formula

Formula weight

Crystallization solvent

Crystal shape

Crystal color

Crystal size
$\mathrm{C}_{52} \mathrm{H}_{60} \mathrm{BNP}_{2} \mathrm{Pd}$

878.16

$\mathrm{CH}_{2} \mathrm{Cl}_{2}$

tabular

colorless

$0.10 \times 0.14 \times 0.15 \mathrm{~mm}$

\section{Data Collection}

Preliminary photograph(s)

Type of diffractometer

Wavelength

Data collection temperature

Theta range for 6911 reflections used in lattice determination

Unit cell dimensions

Volume

Z

Crystal system

Space group

Density (calculated)

$\mathrm{F}(000)$

Theta range for data collection

Completeness to theta $=28.50^{\circ}$

Index ranges

Data collection scan type

Reflections collected

Independent reflections

Reflections $>2 \sigma(\mathrm{I})$

Average $\sigma(\mathrm{I}) /($ net I)

Absorption coefficient

Absorption correction

Max. and min. transmission

Reflections monitored for decay

Decay of standards rotation

Bruker SMART $1000 \mathrm{ccd}$

$0.71073 \AA \mathrm{MoK} \alpha$

$98 \mathrm{~K}$

2.40 to $28.67^{\circ}$

$\mathrm{a}=12.9776(11) \AA$

$\alpha=90^{\circ}$

$\mathrm{b}=19.4418(16) \AA$

$\beta=107.570(1)^{\circ}$

$\mathrm{c}=18.1205(15) \AA$

$\gamma=90^{\circ}$
4358.7(6) $\AA^{3}$

4

monoclinic

$P 21 /$ c (\# 14)

$1.338 \mathrm{~g} / \mathrm{cm}^{3}$

1840

1.6 to $28.5^{\circ}$

$94.1 \%$

$-17 \leq \mathrm{h} \leq 17,-26 \leq \mathrm{k} \leq 25,-23 \leq 1 \leq 24$

$\omega$ scans at 7 fixed $\phi$ values

77250

$10404\left[\mathrm{R}_{\mathrm{int}}=0.0721\right]$

7523

0.0550

$0.54 \mathrm{~mm}^{-1}$

integration

0.9604 and 0.9191

first 75 scans recollected at end of runs

$0 \%$ 


\section{Structure Solution and Refinement}

Primary solution method

Secondary solution method

Hydrogen placement

Refinement method

Data / restraints / parameters

Treatment of hydrogen atoms

Goodness-of-fit on $\mathrm{F}^{2}$

Final $\mathrm{R}$ indices [I $>2 \sigma(\mathrm{I}), 7523$ reflections]

$\mathrm{R}$ indices (all data)

Type of weighting scheme used

Weighting scheme used

Max shift/error

Average shift/error

Largest diff. peak and hole direct methods

difference map

calculated

full-matrix least-squares on $\mathrm{F}^{2}$

10404 / 0 / 515

not refined, $\mathrm{U}_{\text {iso }}$ fixed at $120 \% \mathrm{U}_{\text {eq }}$ of attached atom

1.75

$\mathrm{R} 1=0.0497, \mathrm{wR} 2=0.0953$

$\mathrm{R} 1=0.0777, \mathrm{wR} 2=0.0987$

calculated weights

$\mathrm{w}=1 /\left[\sigma^{2}\left(\mathrm{~F}_{\mathrm{o}}{ }^{2}\right)+(0.02 \mathrm{P})^{2}\right]$ where $\mathrm{P}=\left(\mathrm{F}_{\mathrm{o}}{ }^{2}+2 \mathrm{~F}_{\mathrm{c}}{ }^{2}\right) / 3$

0.027

0.001

4.14 and $-1.68 \mathrm{e} \cdot \AA^{-3}$

\section{Programs Used}

Cell refinement

Bruker SMART v5.606, Bruker SAINT v6.29

Data collection

Bruker SMART v5.054

Data reduction

Bruker SAINT v6.29

Structure solution

SHELX-97 (Sheldrick, 1997)

Structure refinement

SHELX-97 (Sheldrick, 1997)

Graphics

Diamond, Bruker SHELXTL v6.12

\section{References}

Bruker (1999) SMART (v5.054), SMART (v5.606), SAINT (v6.29) and SHELXTL (v6.12). Bruker AXS Inc., Madison, Wisconsin, USA.

Diamond 2.1. (2000) Crystal Impact GbR, Bonn, Germany.

Spek, A.L. (1990). Acta Cryst., A46, C-34.

Sheldrick, G. M. (1997). SHELX-97. Program for Structures Refinement. Univ. of Gottingen, Federal Republic of Germany. 


\section{Special Refinement Details}

A small clear tabular crystal was selected and mounted on a glass fiber with Paratone-N oil. Seven runs of data were collected with 30 second long, $-0.3^{\circ}$ wide $\omega$-scans at six values of $\varphi\left(0,51,103,154,206\right.$, and $\left.309^{\circ}\right)$ with the detector $5 \mathrm{~cm}$ (nominal) distant at a $\theta$ of $-28^{\circ}$. The initial cell for data reduction was calculated from 999 centered reflections chosen from throughout the data frames. A total of 2 reflections was discarded in the triclinic leastsquares with a reciprocal lattice vector tolerance of 0.005. For data processing with SAINT v6.29, all defaults were used, except: a fixed box size of $1.8 \times 1.8 \times 0.7$ was used, periodic orientation matrix updating was disabled, the instrument error was set to zero, no Laue class integration restraints were used, the I/ $\sigma$ lower limit and threshold were set to 5 and 4 respectively, and for the post-integration global least squares refinement, no constraints were applied. No decay correction was needed. A face-indexed absorption correction resulted in marginal improvement.

No reflections were specifically omitted from the final processed dataset; 1207 reflections were rejected, with 0 space group-absence violations, 23 inconsistent equivalents, and no reflections suppressed. Refinement of $\mathrm{F}^{2}$ was against all reflections. The weighted R-factor $(w \mathrm{R})$ and goodness of fit $(\mathrm{S})$ are based on $\mathrm{F}^{2}$, conventional $\mathrm{R}$-factors (R) are based on F, with $F$ set to zero for negative $F^{2}$. The threshold expression of $F^{2}>2 \sigma\left(F^{2}\right)$ is used only for calculating R-factors(gt) etc. and is not relevant to the choice of reflections for refinement.

Of the twenty largest peaks in the final difference map, four are greater than $|1| \mathrm{e} \cdot \AA^{-3}$. Three of these four peaks are near the palladium atom, including the largest positive peak of $4.14 \mathrm{e} \cdot \AA^{-3}$ at a distance of $0.91 \AA$. The largest negative peak of $-1.68 \mathrm{e} \cdot \AA^{-3}$ is $0.58 \AA$ from $\mathrm{Pd}$. 
Table 12. Atomic coordinates $\left(\mathrm{x}_{10}{ }^{4}\right)$ and equivalent isotropic displacement parameters $\left(\AA^{2} \times 10^{3}\right)$ for 8 (CCDC 186799). U(eq) is defined as one third of the trace of the orthogonalized $U^{i j}$ tensor.

\begin{tabular}{|c|c|c|c|c|}
\hline & $\mathrm{x}$ & $\mathrm{y}$ & $\mathrm{z}$ & $\mathrm{U}_{\text {eq }}$ \\
\hline $\mathrm{Pd}$ & $3278.5(2)$ & $8621.2(1)$ & $7521.3(1)$ & $18.0(1)$ \\
\hline $\mathrm{P}(1)$ & $1636.8(7)$ & $9089.6(4)$ & $7605.7(5)$ & $16.7(2)$ \\
\hline $\mathrm{P}(2)$ & $3684.4(7)$ & $9559.6(4)$ & $6932.3(5)$ & $17.3(2)$ \\
\hline $\mathrm{N}$ & $3772(2)$ & $7569(1)$ & $7870(2)$ & $18(1)$ \\
\hline B & $2425(3)$ & $10534(2)$ & $7648(2)$ & $18(1)$ \\
\hline$C(1)$ & $1423(2)$ & $9977(2)$ & $7288(2)$ & $17(1)$ \\
\hline$C(2)$ & $3568(3)$ & $10353(2)$ & $7418(2)$ & $20(1)$ \\
\hline$C(3)$ & 2054(3) & $11301(2)$ & $7290(2)$ & $19(1)$ \\
\hline $\mathrm{C}(4)$ & $1058(3)$ & $11470(2)$ & $6778(2)$ & $26(1)$ \\
\hline$C(5)$ & $810(3)$ & $12129(2)$ & $6481(2)$ & $33(1)$ \\
\hline$C(6)$ & $1576(3)$ & $12639(2)$ & 6671(2) & $35(1)$ \\
\hline$C(7)$ & $2575(3)$ & $12496(2)$ & 7192(2) & $37(1)$ \\
\hline$C(8)$ & 2795(3) & $11837(2)$ & $7498(2)$ & $30(1)$ \\
\hline C(9) & $2670(2)$ & $10538(2)$ & $8589(2)$ & $18(1)$ \\
\hline$C(10)$ & $3396(3)$ & $10096(2)$ & $9089(2)$ & $25(1)$ \\
\hline$C(11)$ & $3518(3)$ & $10060(2)$ & $9875(2)$ & $34(1)$ \\
\hline$C(12)$ & 2911(3) & $10488(2)$ & $10189(2)$ & $35(1)$ \\
\hline$C(13)$ & 2196(3) & $10939(2)$ & $9723(2)$ & $30(1)$ \\
\hline$C(14)$ & 2077(3) & $10966(2)$ & $8935(2)$ & $24(1)$ \\
\hline$C(15)$ & 1233(3) & 8999(2) & $8483(2)$ & $20(1)$ \\
\hline$C(16)$ & 1687(3) & $8473(2)$ & $9001(2)$ & $24(1)$ \\
\hline$C(17)$ & 1336(3) & $8348(2)$ & $9641(2)$ & $29(1)$ \\
\hline$C(18)$ & $535(3)$ & $8750(2)$ & 9771(2) & $29(1)$ \\
\hline$C(19)$ & 91(3) & $9283(2)$ & $9277(2)$ & $30(1)$ \\
\hline$C(20)$ & $443(3)$ & $9404(2)$ & $8633(2)$ & $26(1)$ \\
\hline $\mathrm{C}(21)$ & $559(2)$ & $8612(2)$ & $6900(2)$ & $19(1)$ \\
\hline $\mathrm{C}(22)$ & $-342(3)$ & $8337(2)$ & $7026(2)$ & $24(1)$ \\
\hline $\mathrm{C}(23)$ & $-1091(3)$ & $7960(2)$ & $6460(2)$ & $31(1)$ \\
\hline $\mathrm{C}(24)$ & $-945(3)$ & $7860(2)$ & $5739(2)$ & $31(1)$ \\
\hline$C(25)$ & $-46(3)$ & $8130(2)$ & $5605(2)$ & $34(1)$ \\
\hline$C(26)$ & 703(3) & $8495(2)$ & $6173(2)$ & $29(1)$ \\
\hline$C(27)$ & 2841(3) & $9642(2)$ & $5928(2)$ & $20(1)$ \\
\hline $\mathrm{C}(28)$ & 2231(3) & $10228(2)$ & $5643(2)$ & $26(1)$ \\
\hline$C(29)$ & $1538(3)$ & $10236(2)$ & 4897(2) & $36(1)$ \\
\hline$C(30)$ & $1440(3)$ & $9679(2)$ & $4425(2)$ & $39(1)$ \\
\hline$C(31)$ & 2053(3) & $9098(2)$ & 4684(2) & $35(1)$ \\
\hline$C(32)$ & 2754(3) & $9079(2)$ & $5437(2)$ & $27(1)$ \\
\hline$C(33)$ & $5055(2)$ & $9560(2)$ & $6838(2)$ & $19(1)$ \\
\hline$C(34)$ & $5288(3)$ & $9752(2)$ & $6169(2)$ & $25(1)$ \\
\hline$C(35)$ & $6329(3)$ & $9720(2)$ & $6125(2)$ & $29(1)$ \\
\hline$C(36)$ & 7154(3) & $9496(2)$ & $6748(2)$ & $28(1)$ \\
\hline$C(37)$ & $6958(3)$ & $9326(2)$ & $7428(2)$ & $28(1)$ \\
\hline$C(38)$ & $5900(3)$ & $9350(2)$ & $7472(2)$ & $23(1)$ \\
\hline$C(39)$ & $4428(3)$ & $7922(2)$ & $7478(2)$ & $22(1)$ \\
\hline $\mathrm{C}(40)$ & 4484(3) & $7687(2)$ & $6698(2)$ & $27(1)$ \\
\hline $\mathrm{C}(41)$ & $4288(3)$ & $7446(2)$ & $8720(2)$ & $24(1)$ \\
\hline
\end{tabular}




\begin{tabular}{lrrrr}
$\mathrm{C}(42)$ & $4665(3)$ & $8108(2)$ & $9166(2)$ & $25(1)$ \\
$\mathrm{C}(43)$ & $5065(3)$ & $7974(2)$ & $10036(2)$ & $34(1)$ \\
$\mathrm{C}(44)$ & $5984(3)$ & $7448(2)$ & $10226(2)$ & $34(1)$ \\
$\mathrm{C}(45)$ & $5656(3)$ & $6792(2)$ & $9764(2)$ & $35(1)$ \\
$\mathrm{C}(46)$ & $5221(3)$ & $6931(2)$ & $8898(2)$ & $32(1)$ \\
$\mathrm{C}(47)$ & $2952(3)$ & $7075(2)$ & $7415(2)$ & $22(1)$ \\
$\mathrm{C}(48)$ & $3371(3)$ & $6347(2)$ & $7328(2)$ & $29(1)$ \\
$\mathrm{C}(49)$ & $2504(3)$ & $5968(2)$ & $6712(2)$ & $37(1)$ \\
$\mathrm{C}(50)$ & $1447(3)$ & $5929(2)$ & $6931(2)$ & $38(1)$ \\
$\mathrm{C}(51)$ & $1071(3)$ & $6633(2)$ & $7102(2)$ & $34(1)$ \\
$\mathrm{C}(52)$ & $1958(3)$ & $7026(2)$ & $7698(2)$ & $26(1)$ \\
\hline
\end{tabular}


Table 13. Bond lengths $[\AA ̊]$ and angles $\left[^{\circ}\right]$ for 8 (CCDC 186799).

\begin{tabular}{|c|c|c|c|}
\hline Pd-C(39) & $2.037(3)$ & $C(19)-C(20)$ & $1.395(5)$ \\
\hline Pd-N & $2.180(3)$ & C(19)-H(19) & 0.9500 \\
\hline Pd-P(2) & $2.2549(9)$ & $\mathrm{C}(20)-\mathrm{H}(20)$ & 0.9500 \\
\hline Pd-P(1) & $2.3641(9)$ & $C(21)-C(22)$ & $1.366(4)$ \\
\hline$P(1)-C(1)$ & $1.813(3)$ & $C(21)-C(26)$ & $1.403(4)$ \\
\hline $\mathrm{P}(1)-\mathrm{C}(15)$ & $1.827(3)$ & $C(22)-C(23)$ & $1.390(5)$ \\
\hline$P(1)-C(21)$ & $1.837(3)$ & $\mathrm{C}(22)-\mathrm{H}(22)$ & 0.9500 \\
\hline $\mathrm{P}(2)-\mathrm{C}(2)$ & $1.806(3)$ & $C(23)-C(24)$ & $1.388(5)$ \\
\hline $\mathrm{P}(2)-\mathrm{C}(27)$ & $1.825(3)$ & C(23)-H(23) & 0.9500 \\
\hline $\mathrm{P}(2)-\mathrm{C}(33)$ & $1.838(3)$ & $C(24)-C(25)$ & $1.366(5)$ \\
\hline N-C(39) & $1.438(4)$ & C(24)-H(24) & 0.9500 \\
\hline $\mathrm{N}-\mathrm{C}(47)$ & $1.484(4)$ & $C(25)-C(26)$ & $1.381(5)$ \\
\hline $\mathrm{N}-\mathrm{C}(41)$ & $1.502(4)$ & $\mathrm{C}(25)-\mathrm{H}(25)$ & 0.9500 \\
\hline B-C(9) & $1.637(5)$ & $\mathrm{C}(26)-\mathrm{H}(26)$ & 0.9500 \\
\hline B-C(3) & $1.639(5)$ & C(27)-C(32) & $1.394(5)$ \\
\hline B-C(1) & $1.666(5)$ & $C(27)-C(28)$ & $1.395(4)$ \\
\hline B-C(2) & $1.694(5)$ & $C(28)-C(29)$ & $1.380(5)$ \\
\hline $\mathrm{C}(1)-\mathrm{H}(1 \mathrm{~A})$ & 0.9900 & $\mathrm{C}(28)-\mathrm{H}(28)$ & 0.9500 \\
\hline C(1)-H(1B) & 0.9900 & C(29)-C(30) & $1.362(6)$ \\
\hline $\mathrm{C}(2)-\mathrm{H}(2 \mathrm{~A})$ & 0.9900 & C(29)-H(29) & 0.9500 \\
\hline $\mathrm{C}(2)-\mathrm{H}(2 \mathrm{~B})$ & 0.9900 & $C(30)-C(31)$ & $1.379(6)$ \\
\hline C(3)-C(4) & $1.384(5)$ & $\mathrm{C}(30)-\mathrm{H}(30)$ & 0.9500 \\
\hline $\mathrm{C}(3)-\mathrm{C}(8)$ & $1.392(5)$ & $C(31)-C(32)$ & $1.393(5)$ \\
\hline $\mathrm{C}(4)-\mathrm{C}(5)$ & $1.388(5)$ & $\mathrm{C}(31)-\mathrm{H}(31)$ & 0.9500 \\
\hline $\mathrm{C}(4)-\mathrm{H}(4)$ & 0.9500 & $\mathrm{C}(32)-\mathrm{H}(32)$ & 0.9500 \\
\hline$C(5)-C(6)$ & $1.373(5)$ & C(33)-C(34) & $1.386(4)$ \\
\hline $\mathrm{C}(5)-\mathrm{H}(5)$ & 0.9500 & C(33)-C(38) & $1.388(4)$ \\
\hline$C(6)-C(7)$ & $1.382(5)$ & C(34)-C(35) & $1.379(5)$ \\
\hline $\mathrm{C}(6)-\mathrm{H}(6)$ & 0.9500 & C(34)-H(34) & 0.9500 \\
\hline$C(7)-C(8)$ & $1.390(5)$ & $C(35)-C(36)$ & $1.371(5)$ \\
\hline $\mathrm{C}(7)-\mathrm{H}(7)$ & 0.9500 & $\mathrm{C}(35)-\mathrm{H}(35)$ & 0.9500 \\
\hline $\mathrm{C}(8)-\mathrm{H}(8)$ & 0.9500 & $C(36)-C(37)$ & $1.371(5)$ \\
\hline$C(9)-C(10)$ & $1.389(4)$ & $\mathrm{C}(36)-\mathrm{H}(36)$ & 0.9500 \\
\hline C(9)-C(14) & $1.403(4)$ & C(37)-C(38) & $1.401(5)$ \\
\hline$C(10)-C(11)$ & $1.387(5)$ & $\mathrm{C}(37)-\mathrm{H}(37)$ & 0.9500 \\
\hline $\mathrm{C}(10)-\mathrm{H}(10)$ & 0.9500 & $\mathrm{C}(38)-\mathrm{H}(38)$ & 0.9500 \\
\hline$C(11)-C(12)$ & $1.382(5)$ & C(39)-C(40) & $1.508(4)$ \\
\hline $\mathrm{C}(11)-\mathrm{H}(11)$ & 0.9500 & C(39)-H(39) & 1.0000 \\
\hline$C(12)-C(13)$ & $1.367(5)$ & $\mathrm{C}(40)-\mathrm{H}(40 \mathrm{~A})$ & 0.9800 \\
\hline $\mathrm{C}(12)-\mathrm{H}(12)$ & 0.9500 & $\mathrm{C}(40)-\mathrm{H}(40 \mathrm{~B})$ & 0.9800 \\
\hline$C(13)-C(14)$ & $1.390(4)$ & $\mathrm{C}(40)-\mathrm{H}(40 \mathrm{C})$ & 0.9800 \\
\hline $\mathrm{C}(13)-\mathrm{H}(13)$ & 0.9500 & $C(41)-C(42)$ & $1.520(5)$ \\
\hline $\mathrm{C}(14)-\mathrm{H}(14)$ & 0.9500 & $C(41)-C(46)$ & $1.529(4)$ \\
\hline$C(15)-C(20)$ & $1.383(4)$ & $\mathrm{C}(41)-\mathrm{H}(41)$ & 1.0000 \\
\hline$C(15)-C(16)$ & $1.393(4)$ & $C(42)-C(43)$ & $1.526(5)$ \\
\hline$C(16)-C(17)$ & $1.390(5)$ & $\mathrm{C}(42)-\mathrm{H}(42 \mathrm{~A})$ & 0.9900 \\
\hline $\mathrm{C}(16)-\mathrm{H}(16)$ & 0.9500 & $\mathrm{C}(42)-\mathrm{H}(42 \mathrm{~B})$ & 0.9900 \\
\hline$C(17)-C(18)$ & $1.377(5)$ & $\mathrm{C}(43)-\mathrm{C}(44)$ & $1.530(5)$ \\
\hline $\mathrm{C}(17)-\mathrm{H}(17)$ & 0.9500 & $\mathrm{C}(43)-\mathrm{H}(43 \mathrm{~A})$ & 0.9900 \\
\hline C(18)-C(19) & $1.376(5)$ & $\mathrm{C}(43)-\mathrm{H}(43 \mathrm{~B})$ & 0.9900 \\
\hline $\mathrm{C}(18)-\mathrm{H}(18)$ & 0.9500 & C(44)-C(45) & $1.513(5)$ \\
\hline
\end{tabular}




\begin{tabular}{|c|c|c|c|}
\hline $\mathrm{C}(44)-\mathrm{H}(44 \mathrm{~A})$ & 0.9900 & $\mathrm{C}(1)-\mathrm{B}-\mathrm{C}(2)$ & $114.0(3)$ \\
\hline $\mathrm{C}(44)-\mathrm{H}(44 \mathrm{~B})$ & 0.9900 & B-C(1)-P(1) & $117.9(2)$ \\
\hline$C(45)-C(46)$ & $1.523(5)$ & $\mathrm{B}-\mathrm{C}(1)-\mathrm{H}(1 \mathrm{~A})$ & 107.8 \\
\hline $\mathrm{C}(45)-\mathrm{H}(45 \mathrm{~A})$ & 0.9900 & $\mathrm{P}(1)-\mathrm{C}(1)-\mathrm{H}(1 \mathrm{~A})$ & 107.8 \\
\hline $\mathrm{C}(45)-\mathrm{H}(45 \mathrm{~B})$ & 0.9900 & $\mathrm{~B}-\mathrm{C}(1)-\mathrm{H}(1 \mathrm{~B})$ & 107.8 \\
\hline $\mathrm{C}(46)-\mathrm{H}(46 \mathrm{~A})$ & 0.9900 & $\mathrm{P}(1)-\mathrm{C}(1)-\mathrm{H}(1 \mathrm{~B})$ & 107.8 \\
\hline $\mathrm{C}(46)-\mathrm{H}(46 \mathrm{~B})$ & 0.9900 & $\mathrm{H}(1 \mathrm{~A})-\mathrm{C}(1)-\mathrm{H}(1 \mathrm{~B})$ & 107.2 \\
\hline $\mathrm{C}(47)-\mathrm{C}(52)$ & $1.528(5)$ & $\mathrm{B}-\mathrm{C}(2)-\mathrm{P}(2)$ & $120.4(2)$ \\
\hline $\mathrm{C}(47)-\mathrm{C}(48)$ & $1.542(4)$ & $\mathrm{B}-\mathrm{C}(2)-\mathrm{H}(2 \mathrm{~A})$ & 107.2 \\
\hline $\mathrm{C}(47)-\mathrm{H}(47)$ & 1.0000 & $\mathrm{P}(2)-\mathrm{C}(2)-\mathrm{H}(2 \mathrm{~A})$ & 107.2 \\
\hline C(48)-C(49) & $1.515(5)$ & $\mathrm{B}-\mathrm{C}(2)-\mathrm{H}(2 \mathrm{~B})$ & 107.2 \\
\hline $\mathrm{C}(48)-\mathrm{H}(48 \mathrm{~A})$ & 0.9900 & $\mathrm{P}(2)-\mathrm{C}(2)-\mathrm{H}(2 \mathrm{~B})$ & 107.2 \\
\hline $\mathrm{C}(48)-\mathrm{H}(48 \mathrm{~B})$ & 0.9900 & $\mathrm{H}(2 \mathrm{~A})-\mathrm{C}(2)-\mathrm{H}(2 \mathrm{~B})$ & 106.9 \\
\hline $\mathrm{C}(49)-\mathrm{C}(50)$ & $1.541(5)$ & $C(4)-C(3)-C(8)$ & $115.6(3)$ \\
\hline C(49)-H(49A) & 0.9900 & $C(4)-C(3)-B$ & $125.9(3)$ \\
\hline C(49)-H(49B) & 0.9900 & $\mathrm{C}(8)-\mathrm{C}(3)-\mathrm{B}$ & $118.5(3)$ \\
\hline $\mathrm{C}(50)-\mathrm{C}(51)$ & $1.514(5)$ & $C(3)-C(4)-C(5)$ & $122.7(3)$ \\
\hline $\mathrm{C}(50)-\mathrm{H}(50 \mathrm{~A})$ & 0.9900 & $\mathrm{C}(3)-\mathrm{C}(4)-\mathrm{H}(4)$ & 118.7 \\
\hline $\mathrm{C}(50)-\mathrm{H}(50 \mathrm{~B})$ & 0.9900 & $\mathrm{C}(5)-\mathrm{C}(4)-\mathrm{H}(4)$ & 118.7 \\
\hline $\mathrm{C}(51)-\mathrm{C}(52)$ & $1.525(5)$ & $C(6)-C(5)-C(4)$ & $120.2(4)$ \\
\hline $\mathrm{C}(51)-\mathrm{H}(51 \mathrm{~A})$ & 0.9900 & $\mathrm{C}(6)-\mathrm{C}(5)-\mathrm{H}(5)$ & 119.9 \\
\hline C(51)-H(51B) & 0.9900 & $\mathrm{C}(4)-\mathrm{C}(5)-\mathrm{H}(5)$ & 119.9 \\
\hline C(52)-H(52A) & 0.9900 & $C(5)-C(6)-C(7)$ & $119.0(3)$ \\
\hline \multirow[t]{2}{*}{$\mathrm{C}(52)-\mathrm{H}(52 \mathrm{~B})$} & 0.9900 & $\mathrm{C}(5)-\mathrm{C}(6)-\mathrm{H}(6)$ & 120.5 \\
\hline & & $\mathrm{C}(7)-\mathrm{C}(6)-\mathrm{H}(6)$ & 120.5 \\
\hline C(39)-Pd-N & $39.70(11)$ & $C(6)-C(7)-C(8)$ & $119.7(4)$ \\
\hline C(39)-Pd-P(2) & 104.04(9) & $\mathrm{C}(6)-\mathrm{C}(7)-\mathrm{H}(7)$ & 120.2 \\
\hline N-Pd-P(2) & $143.75(7)$ & $\mathrm{C}(8)-\mathrm{C}(7)-\mathrm{H}(7)$ & 120.2 \\
\hline C(39)-Pd-P(1) & $160.81(9)$ & $C(7)-C(8)-C(3)$ & $122.7(4)$ \\
\hline N-Pd-P(1) & $122.31(7)$ & $\mathrm{C}(7)-\mathrm{C}(8)-\mathrm{H}(8)$ & 118.7 \\
\hline P(2)-Pd-P(1) & $93.60(3)$ & $\mathrm{C}(3)-\mathrm{C}(8)-\mathrm{H}(8)$ & 118.7 \\
\hline C(1)-P(1)-C(15) & $108.13(15)$ & C(10)-C(9)-C(14) & $115.5(3)$ \\
\hline C(1)-P(1)-C(21) & $104.40(14)$ & C(10)-C(9)-B & $123.8(3)$ \\
\hline C(15)-P(1)-C(21) & $101.48(14)$ & C(14)-C(9)-B & $120.5(3)$ \\
\hline C(1)-P(1)-Pd & $113.62(10)$ & $\mathrm{C}(11)-\mathrm{C}(10)-\mathrm{C}(9)$ & 123.1(3) \\
\hline $\mathrm{C}(15)-\mathrm{P}(1)-\mathrm{Pd}$ & 121.34(11) & $\mathrm{C}(11)-\mathrm{C}(10)-\mathrm{H}(10)$ & 118.4 \\
\hline C(21)-P(1)-Pd & $105.77(10)$ & $\mathrm{C}(9)-\mathrm{C}(10)-\mathrm{H}(10)$ & 118.4 \\
\hline C(2)-P(2)-C(27) & $107.36(15)$ & $\mathrm{C}(12)-\mathrm{C}(11)-\mathrm{C}(10)$ & 119.3(3) \\
\hline C(2)-P(2)-C(33) & $105.75(14)$ & $\mathrm{C}(12)-\mathrm{C}(11)-\mathrm{H}(11)$ & 120.4 \\
\hline C(27)-P(2)-C(33) & $102.31(14)$ & $\mathrm{C}(10)-\mathrm{C}(11)-\mathrm{H}(11)$ & 120.4 \\
\hline C(2)-P(2)-Pd & $113.23(11)$ & $\mathrm{C}(13)-\mathrm{C}(12)-\mathrm{C}(11)$ & $119.8(3)$ \\
\hline $\mathrm{C}(27)-\mathrm{P}(2)-\mathrm{Pd}$ & $112.79(11)$ & $\mathrm{C}(13)-\mathrm{C}(12)-\mathrm{H}(12)$ & 120.1 \\
\hline C(33)-P(2)-Pd & 114.51(11) & $\mathrm{C}(11)-\mathrm{C}(12)-\mathrm{H}(12)$ & 120.1 \\
\hline C(39)-N-C(47) & $117.9(3)$ & $\mathrm{C}(12)-\mathrm{C}(13)-\mathrm{C}(14)$ & $120.2(3)$ \\
\hline C(39)-N-C(41) & $115.8(3)$ & $\mathrm{C}(12)-\mathrm{C}(13)-\mathrm{H}(13)$ & 119.9 \\
\hline C(47)-N-C(41) & $119.3(2)$ & $\mathrm{C}(14)-\mathrm{C}(13)-\mathrm{H}(13)$ & 119.9 \\
\hline $\mathrm{C}(39)-\mathrm{N}-\mathrm{Pd}$ & $64.78(16)$ & $\mathrm{C}(13)-\mathrm{C}(14)-\mathrm{C}(9)$ & $122.1(3)$ \\
\hline C(47)-N-Pd & $110.33(18)$ & $\mathrm{C}(13)-\mathrm{C}(14)-\mathrm{H}(14)$ & 119.0 \\
\hline C(41)-N-Pd & $116.58(19)$ & $\mathrm{C}(9)-\mathrm{C}(14)-\mathrm{H}(14)$ & 119.0 \\
\hline $\mathrm{C}(9)-\mathrm{B}-\mathrm{C}(3)$ & $110.5(3)$ & $C(20)-C(15)-C(16)$ & $118.3(3)$ \\
\hline $\mathrm{C}(9)-\mathrm{B}-\mathrm{C}(1)$ & $107.0(3)$ & $\mathrm{C}(20)-\mathrm{C}(15)-\mathrm{P}(1)$ & $122.7(3)$ \\
\hline $\mathrm{C}(3)-\mathrm{B}-\mathrm{C}(1)$ & 109.6(3) & $\mathrm{C}(16)-\mathrm{C}(15)-\mathrm{P}(1)$ & $118.9(2)$ \\
\hline $\mathrm{C}(9)-\mathrm{B}-\mathrm{C}(2)$ & $110.3(3)$ & $C(17)-C(16)-C(15)$ & $120.8(3)$ \\
\hline $\mathrm{C}(3)-\mathrm{B}-\mathrm{C}(2)$ & $105.4(3)$ & $\mathrm{C}(17)-\mathrm{C}(16)-\mathrm{H}(16)$ & 119.6 \\
\hline
\end{tabular}




$\begin{array}{ll}\mathrm{C}(15)-\mathrm{C}(16)-\mathrm{H}(16) & 119.6 \\ \mathrm{C}(18)-\mathrm{C}(17)-\mathrm{C}(16) & 119.8(3) \\ \mathrm{C}(18)-\mathrm{C}(17)-\mathrm{H}(17) & 120.1 \\ \mathrm{C}(16)-\mathrm{C}(17)-\mathrm{H}(17) & 120.1 \\ \mathrm{C}(19)-\mathrm{C}(18)-\mathrm{C}(17) & 120.4(3) \\ \mathrm{C}(19)-\mathrm{C}(18)-\mathrm{H}(18) & 119.8 \\ \mathrm{C}(17)-\mathrm{C}(18)-\mathrm{H}(18) & 119.8 \\ \mathrm{C}(18)-\mathrm{C}(19)-\mathrm{C}(20) & 119.5(3) \\ \mathrm{C}(18)-\mathrm{C}(19)-\mathrm{H}(19) & 120.2 \\ \mathrm{C}(20)-\mathrm{C}(19)-\mathrm{H}(19) & 120.2 \\ \mathrm{C}(15)-\mathrm{C}(20)-\mathrm{C}(19) & 121.1(3) \\ \mathrm{C}(15)-\mathrm{C}(20)-\mathrm{H}(20) & 119.4 \\ \mathrm{C}(19)-\mathrm{C}(20)-\mathrm{H}(20) & 119.4 \\ \mathrm{C}(22)-\mathrm{C}(21)-\mathrm{C}(26) & 117.1(3) \\ \mathrm{C}(22)-\mathrm{C}(21)-\mathrm{P}(1) & 126.3(2) \\ \mathrm{C}(26)-\mathrm{C}(21)-\mathrm{P}(1) & 116.5(2) \\ \mathrm{C}(21)-\mathrm{C}(22)-\mathrm{C}(23) & 121.7(3) \\ \mathrm{C}(21)-\mathrm{C}(22)-\mathrm{H}(22) & 119.1 \\ \mathrm{C}(23)-\mathrm{C}(22)-\mathrm{H}(22) & 119.1 \\ \mathrm{C}(24)-\mathrm{C}(23)-\mathrm{C}(22) & 120.3(3) \\ \mathrm{C}(24)-\mathrm{C}(23)-\mathrm{H}(23) & 119.9 \\ \mathrm{C}(22)-\mathrm{C}(23)-\mathrm{H}(23) & 119.9 \\ \mathrm{C}(25)-\mathrm{C}(24)-\mathrm{C}(23) & 118.7(3) \\ \mathrm{C}(25)-\mathrm{C}(24)-\mathrm{H}(24) & 120.6 \\ \mathrm{C}(23)-\mathrm{C}(24)-\mathrm{H}(24) & 120.6 \\ \mathrm{C}(24)-\mathrm{C}(25)-\mathrm{C}(26) & 120.7(3) \\ \mathrm{C}(24)-\mathrm{C}(25)-\mathrm{H}(25) & 119.7 \\ \mathrm{C}(26)-\mathrm{C}(25)-\mathrm{H}(25) & 119.7 \\ \mathrm{C}(25)-\mathrm{C}(26)-\mathrm{C}(21) & 121.4(3) \\ \mathrm{C}(25)-\mathrm{C}(26)-\mathrm{H}(26) & 119.3 \\ \mathrm{C}(21)-\mathrm{C}(26)-\mathrm{H}(26) & 119.3 \\ \mathrm{C}(32)-\mathrm{C}(27)-\mathrm{C}(28) & 118.6(3) \\ \mathrm{C}(32)-\mathrm{C}(27)-\mathrm{P}(2) & 118.5(3) \\ \mathrm{C}(28)-\mathrm{C}(27)-\mathrm{P}(2) & 122.9(3) \\ \mathrm{C}(29)-\mathrm{C}(28)-\mathrm{C}(27) & 120.0(4) \\ \mathrm{C}(29)-\mathrm{C}(28)-\mathrm{H}(28) & 120.0 \\ \mathrm{C}(27)-\mathrm{C}(28)-\mathrm{H}(28) & 120.0 \\ \mathrm{C}(30)-\mathrm{C}(29)-\mathrm{C}(28) & 121.1(4) \\ \mathrm{C}(30)-\mathrm{C}(29)-\mathrm{H}(29) & 119.4 \\ \mathrm{C}(28)-\mathrm{C}(29)-\mathrm{H}(29) & 119.4 \\ \mathrm{C}(29)-\mathrm{C}(30)-\mathrm{C}(31) & 120.2(4) \\ \mathrm{C}(29)-\mathrm{C}(30)-\mathrm{H}(30) & 119.9 \\ \mathrm{C}(31)-\mathrm{C}(30)-\mathrm{H}(30) & 119.9 \\ \mathrm{C}(30)-\mathrm{C}(31)-\mathrm{C}(32) & 119.6(4) \\ \mathrm{C}(30)-\mathrm{C}(31)-\mathrm{H}(31) & 120.2 \\ \mathrm{C}(32)-\mathrm{C}(31)-\mathrm{H}(31) & 120.2 \\ \mathrm{C}(31)-\mathrm{C}(32)-\mathrm{C}(27) & 120.5(4) \\ \mathrm{C}(31)-\mathrm{C}(32)-\mathrm{H}(32) & 119.7 \\ \mathrm{C}(27)-\mathrm{C}(32)-\mathrm{H}(32) & 119.7 \\ \mathrm{C}(34)-\mathrm{C}(33)-\mathrm{C}(38) & 118.5(3) \\ \mathrm{C}(34)-\mathrm{C}(33)-\mathrm{P}(2) & 123.6(3) \\ \mathrm{C}(38)-\mathrm{C}(33)-\mathrm{P}(2) & \\ \mathrm{C}(35)-\mathrm{C}(34)-\mathrm{C}(33) & \\ \mathrm{C}(35)-\mathrm{C}(34)-\mathrm{H}(34) & \\ & \end{array}$

$\begin{array}{ll}\mathrm{C}(33)-\mathrm{C}(34)-\mathrm{H}(34) & 119.5 \\ \mathrm{C}(36)-\mathrm{C}(35)-\mathrm{C}(34) & 120.2(3) \\ \mathrm{C}(36)-\mathrm{C}(35)-\mathrm{H}(35) & 119.9 \\ \mathrm{C}(34)-\mathrm{C}(35)-\mathrm{H}(35) & 119.9 \\ \mathrm{C}(35)-\mathrm{C}(36)-\mathrm{C}(37) & 120.4(3) \\ \mathrm{C}(35)-\mathrm{C}(36)-\mathrm{H}(36) & 119.8 \\ \mathrm{C}(37)-\mathrm{C}(36)-\mathrm{H}(36) & 119.8 \\ \mathrm{C}(36)-\mathrm{C}(37)-\mathrm{C}(38) & 119.6(3) \\ \mathrm{C}(36)-\mathrm{C}(37)-\mathrm{H}(37) & 120.2 \\ \mathrm{C}(38)-\mathrm{C}(37)-\mathrm{H}(37) & 120.2 \\ \mathrm{C}(33)-\mathrm{C}(38)-\mathrm{C}(37) & 120.3(3) \\ \mathrm{C}(33)-\mathrm{C}(38)-\mathrm{H}(38) & 119.8 \\ \mathrm{C}(37)-\mathrm{C}(38)-\mathrm{H}(38) & 119.8 \\ \mathrm{~N}-\mathrm{C}(39)-\mathrm{C}(40) & 121.4(3) \\ \mathrm{N}-\mathrm{C}(39)-\mathrm{Pd} & 75.51(17) \\ \mathrm{C}(40)-\mathrm{C}(39)-\mathrm{Pd} & 118.8(2) \\ \mathrm{N}-\mathrm{C}(39)-\mathrm{H}(39) & 112.1 \\ \mathrm{C}(40)-\mathrm{C}(39)-\mathrm{H}(39) & 112.1 \\ \mathrm{Pd}-\mathrm{C}(39)-\mathrm{H}(39) & 112.1 \\ \mathrm{C}(39)-\mathrm{C}(40)-\mathrm{H}(40 \mathrm{~A}) & 109.5 \\ \mathrm{C}(39)-\mathrm{C}(40)-\mathrm{H}(40 \mathrm{~B}) & 109.5 \\ \mathrm{H}(40 \mathrm{~A})-\mathrm{C}(40)-\mathrm{H}(40 \mathrm{~B}) & 109.5 \\ \mathrm{C}(39)-\mathrm{C}(40)-\mathrm{H}(40 \mathrm{C}) & 109.5 \\ \mathrm{H}(40 \mathrm{~A})-\mathrm{C}(40)-\mathrm{H}(40 \mathrm{C}) & 109.5 \\ \mathrm{H}(40 \mathrm{~B})-\mathrm{C}(40)-\mathrm{H}(40 \mathrm{C}) & 109.5 \\ \text { N-C(41)-C(42) } & 112.5(3) \\ \mathrm{N}-\mathrm{C}(41)-\mathrm{C}(46) & 113.6(3) \\ \mathrm{C}(42)-\mathrm{C}(41)-\mathrm{C}(46) & 109.6(3) \\ \text { N-C(41)-H(41) } & 106.9 \\ \mathrm{C}(42)-\mathrm{C}(41)-\mathrm{H}(41) & 106.9 \\ \mathrm{C}(46)-\mathrm{C}(41)-\mathrm{H}(41) & 106.9 \\ \mathrm{C}(41)-\mathrm{C}(42)-\mathrm{C}(43) & 111.1(3) \\ \mathrm{C}(41)-\mathrm{C}(42)-\mathrm{H}(42 \mathrm{~A}) & 109.4 \\ \mathrm{C}(43)-\mathrm{C}(42)-\mathrm{H}(42 \mathrm{~A}) & 109.4 \\ \mathrm{C}(41)-\mathrm{C}(42)-\mathrm{H}(42 \mathrm{~B}) & 109.4 \\ \mathrm{C}(43)-\mathrm{C}(42)-\mathrm{H}(42 \mathrm{~B}) & 109.4 \\ \mathrm{H}(42 \mathrm{~A})-\mathrm{C}(42)-\mathrm{H}(42 \mathrm{~B}) & 108.0 \\ \mathrm{C}(42)-\mathrm{C}(43)-\mathrm{C}(44) & 110.2(3) \\ \mathrm{C}(42)-\mathrm{C}(43)-\mathrm{H}(43 \mathrm{~A}) & 109.6 \\ \mathrm{C}(44)-\mathrm{C}(43)-\mathrm{H}(43 \mathrm{~A}) & 109.6 \\ \mathrm{C}(42)-\mathrm{C}(43)-\mathrm{H}(43 \mathrm{~B}) & 109.6 \\ \mathrm{C}(44)-\mathrm{C}(43)-\mathrm{H}(43 \mathrm{~B}) & 109.6 \\ \mathrm{H}(43 \mathrm{~A})-\mathrm{C}(43)-\mathrm{H}(43 \mathrm{~B}) & 108.1 \\ \mathrm{C}(45)-\mathrm{C}(44)-\mathrm{C}(43) & 111.7(3) \\ \mathrm{C}(45)-\mathrm{C}(44)-\mathrm{H}(44 \mathrm{~A}) & 109.3 \\ \mathrm{C}(43)-\mathrm{C}(44)-\mathrm{H}(44 \mathrm{~A}) & 109.3 \\ \mathrm{C}(45)-\mathrm{C}(44)-\mathrm{H}(44 \mathrm{~B}) & 109.3 \\ \mathrm{C}(43)-\mathrm{C}(44)-\mathrm{H}(44 \mathrm{~B}) & 109.3 \\ \mathrm{H}(44 \mathrm{~A})-\mathrm{C}(44)-\mathrm{H}(44 \mathrm{~B}) & 107.9 \\ \mathrm{C}(44)-\mathrm{C}(45)-\mathrm{C}(46) & 112.1(3) \\ \mathrm{C}(44)-\mathrm{C}(45)-\mathrm{H}(45 \mathrm{~A}) & 109.2 \\ \mathrm{C}(46)-\mathrm{C}(45)-\mathrm{H}(45 \mathrm{~A}) & 109.2 \\ \mathrm{C}(44)-\mathrm{C}(45)-\mathrm{H}(45 \mathrm{~B}) & 109.2 \\ \mathrm{C}(46)-\mathrm{C}(45)-\mathrm{H}(45 \mathrm{~B}) & 109.2 \\ & \end{array}$




$\begin{array}{llll}\mathrm{H}(45 \mathrm{~A})-\mathrm{C}(45)-\mathrm{H}(45 \mathrm{~B}) & 107.9 & \mathrm{C}(48)-\mathrm{C}(49)-\mathrm{H}(49 \mathrm{~B}) & 109.5 \\ \mathrm{C}(45)-\mathrm{C}(46)-\mathrm{C}(41) & 110.9(3) & \mathrm{C}(50)-\mathrm{C}(49)-\mathrm{H}(49 \mathrm{~B}) & 109.5 \\ \mathrm{C}(45)-\mathrm{C}(46)-\mathrm{H}(46 \mathrm{~A}) & 109.5 & \mathrm{H}(49 \mathrm{~A})-\mathrm{C}(49)-\mathrm{H}(49 \mathrm{~B}) & 108.1 \\ \mathrm{C}(41)-\mathrm{C}(46)-\mathrm{H}(46 \mathrm{~A}) & 109.5 & \mathrm{C}(51)-\mathrm{C}(50)-\mathrm{C}(49) & 112.0(3) \\ \mathrm{C}(45)-\mathrm{C}(46)-\mathrm{H}(46 \mathrm{~B}) & 109.5 & \mathrm{C}(51)-\mathrm{C}(50)-\mathrm{H}(50 \mathrm{~A}) & 109.2 \\ \mathrm{C}(41)-\mathrm{C}(46)-\mathrm{H}(46 \mathrm{~B}) & 109.5 & \mathrm{C}(49)-\mathrm{C}(50)-\mathrm{H}(50 \mathrm{~A}) & 109.2 \\ \mathrm{H}(46 \mathrm{~A})-\mathrm{C}(46)-\mathrm{H}(46 \mathrm{~B}) & 108.0 & \mathrm{C}(51)-\mathrm{C}(50)-\mathrm{H}(50 \mathrm{~B}) & 109.2 \\ \mathrm{~N}-\mathrm{C}(47)-\mathrm{C}(52) & 113.0(3) & \mathrm{C}(49)-\mathrm{C}(50)-\mathrm{H}(50 \mathrm{~B}) & 109.2 \\ \mathrm{~N}-\mathrm{C}(47)-\mathrm{C}(48) & 115.8(3) & \mathrm{H}(50 \mathrm{~A})-\mathrm{C}(50)-\mathrm{H}(50 \mathrm{~B}) & 107.9 \\ \mathrm{C}(52)-\mathrm{C}(47)-\mathrm{C}(48) & 109.7(3) & \mathrm{C}(50)-\mathrm{C}(51)-\mathrm{C}(52) & 112.1(3) \\ \mathrm{N}-\mathrm{C}(47)-\mathrm{H}(47) & 105.9 & \mathrm{C}(50)-\mathrm{C}(51)-\mathrm{H}(51 \mathrm{~A}) & 109.2 \\ \mathrm{C}(52)-\mathrm{C}(47)-\mathrm{H}(47) & 105.9 & \mathrm{C}(52)-\mathrm{C}(51)-\mathrm{H}(51 \mathrm{~A}) & 109.2 \\ \mathrm{C}(48)-\mathrm{C}(47)-\mathrm{H}(47) & 105.9 & \mathrm{C}(50)-\mathrm{C}(51)-\mathrm{H}(51 \mathrm{~B}) & 109.2 \\ \mathrm{C}(49)-\mathrm{C}(48)-\mathrm{C}(47) & 108.3(3) & \mathrm{C}(52)-\mathrm{C}(51)-\mathrm{H}(51 \mathrm{~B}) & 109.2 \\ \mathrm{C}(49)-\mathrm{C}(48)-\mathrm{H}(48 \mathrm{~A}) & 110.0 & \mathrm{H}(51 \mathrm{~A})-\mathrm{C}(51)-\mathrm{H}(51 \mathrm{~B}) & 107.9 \\ \mathrm{C}(47)-\mathrm{C}(48)-\mathrm{H}(48 \mathrm{~A}) & 110.0 & \mathrm{C}(51)-\mathrm{C}(52)-\mathrm{C}(47) & 109.1(3) \\ \mathrm{C}(49)-\mathrm{C}(48)-\mathrm{H}(48 \mathrm{~B}) & 110.0 & \mathrm{C}(51)-\mathrm{C}(52)-\mathrm{H}(52 \mathrm{~A}) & 109.9 \\ \mathrm{C}(47)-\mathrm{C}(48)-\mathrm{H}(48 \mathrm{~B}) & 110.0 & \mathrm{C}(47)-\mathrm{C}(52)-\mathrm{H}(52 \mathrm{~A}) & 109.9 \\ \mathrm{H}(48 \mathrm{~A})-\mathrm{C}(48)-\mathrm{H}(48 \mathrm{~B}) & 108.4 & \mathrm{C}(51)-\mathrm{C}(52)-\mathrm{H}(52 \mathrm{~B}) & 109.9 \\ \mathrm{C}(48)-\mathrm{C}(49)-\mathrm{C}(50) & 110.8(3) & \mathrm{C}(47)-\mathrm{C}(52)-\mathrm{H}(52 \mathrm{~B}) & 109.9 \\ \mathrm{C}(48)-\mathrm{C}(49)-\mathrm{H}(49 \mathrm{~A}) & 109.5 & \mathrm{H}(52 \mathrm{~A})-\mathrm{C}(52)-\mathrm{H}(52 \mathrm{~B}) & 108.3 \\ \mathrm{C}(50)-\mathrm{C}(49)-\mathrm{H}(49 \mathrm{~A}) & 109.5 & & \end{array}$


Table 14. Anisotropic displacement parameters $\left(\AA^{2} \times 10^{4}\right)$ for 8 (CCDC 186799). The anisotropic displacement factor exponent takes the form: $-2 \pi^{2}\left[h^{2} a *{ }^{2} U^{11}+\ldots+2 h k a * b *\right.$ $\mathbf{U}^{12}$ ]

\begin{tabular}{|c|c|c|c|c|c|c|}
\hline & $\mathrm{U}^{11}$ & $\mathrm{U}^{22}$ & $\mathrm{U}^{33}$ & $\mathrm{U}^{23}$ & $\mathrm{U}^{13}$ & $\mathrm{U}^{12}$ \\
\hline $\mathrm{Pd}$ & $167(1)$ & $178(1)$ & 191(1) & $-3(1)$ & $50(1)$ & $36(1)$ \\
\hline $\mathrm{P}(1)$ & $159(4)$ & $167(4)$ & $179(5)$ & $-7(3)$ & $57(3)$ & $-1(3)$ \\
\hline $\mathrm{P}(2)$ & 134(4) & 201(4) & $189(4)$ & $-19(3)$ & $57(4)$ & $17(3)$ \\
\hline $\mathrm{N}$ & $145(14)$ & 199(14) & $188(15)$ & $18(11)$ & $48(12)$ & $21(11)$ \\
\hline B & $140(18)$ & $160(17)$ & $230(20)$ & $-6(15)$ & $59(16)$ & $17(14)$ \\
\hline $\mathrm{C}(1)$ & $147(16)$ & 193(16) & $170(17)$ & $-2(13)$ & $46(13)$ & $42(12)$ \\
\hline$C(2)$ & $175(17)$ & $190(16)$ & $246(19)$ & $-35(14)$ & $88(15)$ & $-13(13)$ \\
\hline $\mathrm{C}(3)$ & $240(17)$ & $200(17)$ & 191(17) & $-15(14)$ & $140(14)$ & $9(14)$ \\
\hline $\mathrm{C}(4)$ & $320(20)$ & $224(18)$ & $213(18)$ & $-38(15)$ & $63(15)$ & $44(15)$ \\
\hline$C(5)$ & $410(20)$ & $340(20)$ & $200(20)$ & $9(16)$ & $54(17)$ & 171(18) \\
\hline$C(6)$ & $560(30)$ & 239(19) & $340(20)$ & $115(17)$ & $270(20)$ & 149(19) \\
\hline$C(7)$ & $370(20)$ & 199(18) & $630(30)$ & $48(18)$ & $300(20)$ & 4(16) \\
\hline $\mathrm{C}(8)$ & 248(19) & $212(18)$ & $460(20)$ & $6(17)$ & $155(18)$ & $21(15)$ \\
\hline$C(9)$ & $163(16)$ & $163(16)$ & $222(18)$ & $-22(13)$ & $47(14)$ & $-42(13)$ \\
\hline$C(10)$ & $262(19)$ & $232(18)$ & $240(20)$ & $-17(15)$ & $48(16)$ & $13(15)$ \\
\hline $\mathrm{C}(11)$ & $300(20)$ & $380(20)$ & $270(20)$ & $59(17)$ & $-12(17)$ & 1(17) \\
\hline$C(12)$ & $390(20)$ & $470(20)$ & $172(19)$ & $2(17)$ & $73(17)$ & $-84(19)$ \\
\hline$C(13)$ & $320(20)$ & $320(20)$ & $290(20)$ & $-39(17)$ & $159(17)$ & $-43(17)$ \\
\hline$C(14)$ & $248(19)$ & $245(18)$ & $245(19)$ & $35(15)$ & $107(15)$ & $5(15)$ \\
\hline$C(15)$ & $214(18)$ & $210(17)$ & $182(17)$ & $-35(14)$ & $61(14)$ & $-57(14)$ \\
\hline$C(16)$ & 204(18) & $300(20)$ & $208(18)$ & $-8(14)$ & $43(15)$ & $-28(14)$ \\
\hline$C(17)$ & $300(20)$ & $340(20)$ & 211(19) & $24(15)$ & $51(16)$ & $-70(16)$ \\
\hline$C(18)$ & $340(20)$ & $380(20)$ & $179(18)$ & $-55(16)$ & $108(16)$ & $-129(17)$ \\
\hline$C(19)$ & $320(20)$ & $310(20)$ & $330(20)$ & $-95(17)$ & 192(18) & $-44(16)$ \\
\hline$C(20)$ & $310(20)$ & $220(18)$ & $260(20)$ & $-6(15)$ & $130(16)$ & $-3(15)$ \\
\hline $\mathrm{C}(21)$ & $165(16)$ & $161(15)$ & $210(17)$ & $-26(14)$ & $24(13)$ & $32(14)$ \\
\hline$C(22)$ & 198(18) & $320(19)$ & $229(19)$ & $-77(15)$ & $87(15)$ & $-10(15)$ \\
\hline$C(23)$ & 204(19) & $370(20)$ & $370(20)$ & $-48(17)$ & $114(17)$ & $-99(16)$ \\
\hline$C(24)$ & $250(20)$ & $320(20)$ & $300(20)$ & $-66(16)$ & $3(17)$ & $-89(16)$ \\
\hline$C(25)$ & $350(20)$ & $430(20)$ & $240(20)$ & $-96(17)$ & $102(17)$ & $-45(18)$ \\
\hline$C(26)$ & $230(19)$ & $380(20)$ & $265(19)$ & $-45(16)$ & $106(16)$ & $-89(16)$ \\
\hline$C(27)$ & $159(17)$ & 293(18) & $175(17)$ & $23(14)$ & $81(14)$ & $-42(14)$ \\
\hline$C(28)$ & 192(18) & $350(20)$ & $280(20)$ & $51(16)$ & $123(16)$ & $33(15)$ \\
\hline$C(29)$ & $240(20)$ & $550(30)$ & $310(20)$ & $170(20)$ & $112(18)$ & 63(18) \\
\hline $\mathrm{C}(30)$ & 178(19) & $710(30)$ & $250(20)$ & $140(20)$ & $21(16)$ & $-107(19)$ \\
\hline$C(31)$ & $350(20)$ & $510(30)$ & $220(20)$ & $-72(18)$ & $120(18)$ & $-210(20)$ \\
\hline$C(32)$ & 264(19) & $310(20)$ & $240(19)$ & 13(16) & $102(16)$ & $-53(16)$ \\
\hline$C(33)$ & $149(16)$ & 185(16) & $244(18)$ & $-37(14)$ & $81(14)$ & $-21(13)$ \\
\hline$C(34)$ & 191(18) & 309(19) & $227(19)$ & $-28(15)$ & $29(15)$ & $-14(15)$ \\
\hline$C(35)$ & $270(20)$ & $380(20)$ & $270(20)$ & $-63(16)$ & 141(17) & $-83(16)$ \\
\hline$C(36)$ & 206(19) & 274(19) & $410(20)$ & $-71(17)$ & $160(17)$ & $-30(15)$ \\
\hline$C(37)$ & $125(18)$ & $210(17)$ & $410(20)$ & $-9(16)$ & $-35(17)$ & $10(13)$ \\
\hline$C(38)$ & 241(19) & 196(17) & $248(19)$ & $-10(14)$ & 71(16) & $-30(14)$ \\
\hline C(39) & 209(18) & $202(17)$ & $290(20)$ & $25(14)$ & $107(15)$ & $38(14)$ \\
\hline $\mathrm{C}(40)$ & $290(20)$ & 288(19) & $290(20)$ & $16(16)$ & $151(17)$ & $53(16)$ \\
\hline
\end{tabular}




\begin{tabular}{llllrrr}
$\mathrm{C}(41)$ & $225(18)$ & $273(18)$ & $203(18)$ & $70(15)$ & $60(15)$ & $30(15)$ \\
$\mathrm{C}(42)$ & $218(19)$ & $280(19)$ & $226(19)$ & $15(15)$ & $22(15)$ & $5(15)$ \\
$\mathrm{C}(43)$ & $330(20)$ & $400(20)$ & $260(20)$ & $14(17)$ & $64(17)$ & $17(17)$ \\
$\mathrm{C}(44)$ & $240(20)$ & $470(20)$ & $260(20)$ & $71(18)$ & $14(17)$ & $41(17)$ \\
$\mathrm{C}(45)$ & $310(20)$ & $390(20)$ & $340(20)$ & $159(18)$ & $64(18)$ & $84(18)$ \\
$\mathrm{C}(46)$ & $290(20)$ & $320(20)$ & $320(20)$ & $45(17)$ & $55(17)$ & $97(16)$ \\
$\mathrm{C}(47)$ & $233(18)$ & $201(17)$ & $219(19)$ & $14(14)$ & $44(15)$ & $-7(14)$ \\
$\mathrm{C}(48)$ & $340(20)$ & $184(16)$ & $330(20)$ & $32(16)$ & $67(16)$ & $8(16)$ \\
$\mathrm{C}(49)$ & $520(30)$ & $192(19)$ & $360(20)$ & $-3(16)$ & $80(20)$ & $6(17)$ \\
$\mathrm{C}(50)$ & $410(20)$ & $290(20)$ & $380(20)$ & $65(18)$ & $23(19)$ & $-82(18)$ \\
$\mathrm{C}(51)$ & $320(20)$ & $350(20)$ & $340(20)$ & $34(17)$ & $91(18)$ & $-97(17)$ \\
$\mathrm{C}(52)$ & $250(20)$ & $281(19)$ & $250(20)$ & $48(15)$ & $78(16)$ & $-32(15)$ \\
& & & & & \\
\hline
\end{tabular}


Table 15. Hydrogen coordinates $\left(x 0^{3}\right)$ and isotropic displacement parameters $\left(\AA^{2} \times 10^{3}\right)$ for 8 (CCDC 186799).

\begin{tabular}{|c|c|c|c|c|}
\hline & $\mathrm{x}$ & $\mathrm{y}$ & z & $U_{\text {iso }}$ \\
\hline $\mathrm{H}(1 \mathrm{~A})$ & 125 & 998 & 672 & 20 \\
\hline $\mathrm{H}(1 \mathrm{~B})$ & 78 & 1015 & 741 & 20 \\
\hline $\mathrm{H}(2 \mathrm{~A})$ & 417 & 1037 & 790 & 24 \\
\hline $\mathrm{H}(2 \mathrm{~B})$ & 368 & 1073 & 709 & 24 \\
\hline $\mathrm{H}(4)$ & 52 & 1112 & 662 & 31 \\
\hline $\mathrm{H}(5)$ & 11 & 1223 & 614 & 39 \\
\hline $\mathrm{H}(6)$ & 142 & 1308 & 645 & 42 \\
\hline $\mathrm{H}(7)$ & 311 & 1284 & 734 & 44 \\
\hline $\mathrm{H}(8)$ & 348 & 1175 & 786 & 35 \\
\hline $\mathrm{H}(10)$ & 383 & 981 & 888 & 30 \\
\hline $\mathrm{H}(11)$ & 401 & 974 & 1019 & 41 \\
\hline $\mathrm{H}(12)$ & 299 & 1047 & 1073 & 42 \\
\hline $\mathrm{H}(13)$ & 178 & 1124 & 994 & 36 \\
\hline $\mathrm{H}(14)$ & 158 & 1128 & 862 & 29 \\
\hline $\mathrm{H}(16)$ & 224 & 820 & 892 & 29 \\
\hline $\mathrm{H}(17)$ & 165 & 798 & 999 & 35 \\
\hline $\mathrm{H}(18)$ & 29 & 866 & 1020 & 35 \\
\hline $\mathrm{H}(19)$ & -45 & 956 & 937 & 36 \\
\hline $\mathrm{H}(20)$ & 13 & 977 & 829 & 31 \\
\hline $\mathrm{H}(22)$ & -46 & 840 & 751 & 29 \\
\hline $\mathrm{H}(23)$ & -170 & 777 & 657 & 37 \\
\hline $\mathrm{H}(24)$ & -146 & 761 & 535 & 37 \\
\hline $\mathrm{H}(25)$ & 6 & 807 & 511 & 40 \\
\hline $\mathrm{H}(26)$ & 133 & 867 & 607 & 34 \\
\hline $\mathrm{H}(28)$ & 229 & 1062 & 596 & 32 \\
\hline $\mathrm{H}(29)$ & 112 & 1064 & 471 & 43 \\
\hline $\mathrm{H}(30)$ & 95 & 969 & 392 & 47 \\
\hline $\mathrm{H}(31)$ & 200 & 871 & 435 & 42 \\
\hline $\mathrm{H}(32)$ & 318 & 868 & 562 & 32 \\
\hline $\mathrm{H}(34)$ & 472 & 991 & 574 & 30 \\
\hline $\mathrm{H}(35)$ & 648 & 985 & 566 & 35 \\
\hline $\mathrm{H}(36)$ & 787 & 946 & 671 & 34 \\
\hline $\mathrm{H}(37)$ & 754 & 919 & 787 & 33 \\
\hline $\mathrm{H}(38)$ & 576 & 922 & 794 & 27 \\
\hline H(39) & 516 & 804 & 784 & 27 \\
\hline $\mathrm{H}(40 \mathrm{~A})$ & 487 & 725 & 675 & 33 \\
\hline $\mathrm{H}(40 \mathrm{~B})$ & 487 & 803 & 649 & 33 \\
\hline $\mathrm{H}(40 \mathrm{C})$ & 375 & 763 & 635 & 33 \\
\hline $\mathrm{H}(41)$ & 372 & 724 & 892 & 28 \\
\hline $\mathrm{H}(42 \mathrm{~A})$ & 406 & 844 & 905 & 30 \\
\hline $\mathrm{H}(42 \mathrm{~B})$ & 526 & 831 & 900 & 30 \\
\hline $\mathrm{H}(43 \mathrm{~A})$ & 446 & 780 & 1021 & 40 \\
\hline $\mathrm{H}(43 \mathrm{~B})$ & 533 & 841 & 1031 & 40 \\
\hline $\mathrm{H}(44 \mathrm{~A})$ & 662 & 765 & 1011 & 40 \\
\hline $\mathrm{H}(44 \mathrm{~B})$ & 620 & 734 & 1078 & 40 \\
\hline $\mathrm{H}(45 \mathrm{~A})$ & 510 & 655 & 994 & 42 \\
\hline $\mathrm{H}(45 \mathrm{~B})$ & 629 & 648 & 987 & 42 \\
\hline
\end{tabular}




$\begin{array}{lrlll}\mathrm{H}(46 \mathrm{~A}) & 497 & 649 & 862 & 38 \\ \mathrm{H}(46 \mathrm{~B}) & 581 & 711 & 871 & 38 \\ \mathrm{H}(47) & 269 & 727 & 688 & 27 \\ \mathrm{H}(48 \mathrm{~A}) & 404 & 638 & 718 & 35 \\ \mathrm{H}(48 \mathrm{~B}) & 354 & 610 & 783 & 35 \\ \mathrm{H}(49 \mathrm{~A}) & 276 & 550 & 665 & 44 \\ \mathrm{H}(49 \mathrm{~B}) & 237 & 621 & 621 & 44 \\ \mathrm{H}(50 \mathrm{~A}) & 88 & 572 & 650 & 46 \\ \mathrm{H}(50 \mathrm{~B}) & 156 & 563 & 739 & 46 \\ \mathrm{H}(51 \mathrm{~A}) & 44 & 658 & 730 & 41 \\ \mathrm{H}(51 \mathrm{~B}) & 84 & 690 & 662 & 41 \\ \mathrm{H}(52 \mathrm{~A}) & 215 & 678 & 820 & 31 \\ \mathrm{H}(52 \mathrm{~B}) & 170 & 749 & 777 & 31\end{array}$


Figure 4. Labeled drawing of 9 (CCDC 186800), with 50\% ellipsoids. Hydrogen atoms have been omitted for clarity.

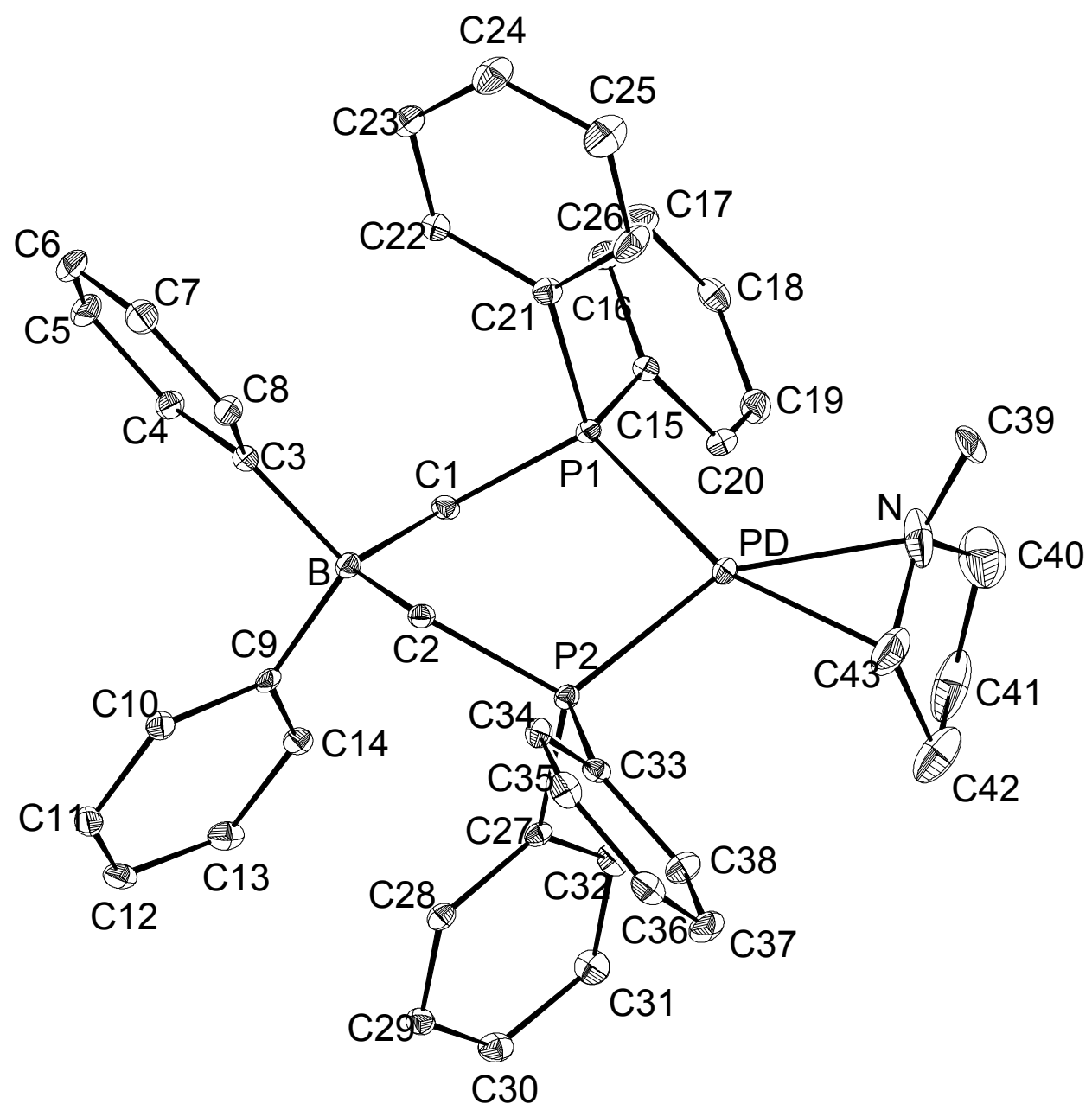


Table 16. Crystal Data and Structure Analysis Details for 9 (CCDC 186800).

Empirical formula

Formula weight

Crystallization solvent

Crystal shape

Crystal color

Crystal size
$\mathrm{C}_{43} \mathrm{H}_{44} \mathrm{BNP}_{2} \mathrm{Pd}$

753.94

tetrahydrofuran

blade

colorless

$0.11 \times 0.12 \times 0.30 \mathrm{~mm}$

\section{Data Collection}

Preliminary photograph(s)

Type of diffractometer

Wavelength

Data collection temperature

Theta range for 8067 reflections used in lattice determination

Unit cell dimensions

Volume

Z

Crystal system

Space group

Density (calculated)

$\mathrm{F}(000)$

Theta range for data collection

Completeness to theta $=28.52^{\circ}$

Index ranges

Data collection scan type

Reflections collected

Independent reflections

Reflections $>2 \sigma(\mathrm{I})$

Average $\sigma(\mathrm{I}) /($ net I)

Absorption coefficient

Absorption correction

Reflections monitored for decay

Decay of standards rotation

Bruker SMART $1000 \mathrm{ccd}$

$0.71073 \AA \mathrm{MoK} \alpha$

$98 \mathrm{~K}$

2.22 to $28.41^{\circ}$

$\mathrm{a}=11.6163(10) \AA$

$\mathrm{b}=29.268(2) \AA$

$\mathrm{c}=11.5305(9) \AA$
$\alpha=90^{\circ}$
$\beta=113.573(1)^{\circ}$
$\gamma=90^{\circ}$

3593.1(5) $\AA^{3}$

4

monoclinic

$P 21 / \mathrm{c} \quad$ (\# 14)

$1.394 \mathrm{~g} / \mathrm{cm}^{3}$

1560

1.4 to $28.5^{\circ}$

$95.0 \%$

$-15 \leq \mathrm{h} \leq 15,-38 \leq \mathrm{k} \leq 38,-15 \leq 1 \leq 15$

$\omega$ scans at 7 fixed $\phi$ values

83659

$8682\left[\mathrm{R}_{\text {int }}=0.0784\right]$

6031

0.060

$0.64 \mathrm{~mm}^{-1}$

none

first 620 scans recollected at end of runs

$0 \%$ 


\section{Structure Solution and Refinement}

Primary solution method

Secondary solution method

Hydrogen placement

Refinement method

Data / restraints / parameters

Treatment of hydrogen atoms

Goodness-of-fit on $\mathrm{F}^{2}$

Final $R$ indices $[\mathrm{I}>2 \sigma(\mathrm{I}), 6031$ reflections]

$\mathrm{R}$ indices (all data)

Type of weighting scheme used

Weighting scheme used

Max shift/error

Average shift/error

Largest diff. peak and hole direct methods

difference map

calculated

full-matrix least-squares on $\mathrm{F}^{2}$

8682 / 0 / 440

not refined, $\mathrm{U}_{\text {iso }}$ fixed at $120 \% \mathrm{U}_{\text {eq }}$ of attached atom

1.61

$\mathrm{R} 1=0.0485, \mathrm{wR} 2=0.0870$

$\mathrm{R} 1=0.0806, \mathrm{wR} 2=0.0900$

calculated weights

calc $\mathrm{w}=1 /\left[\sigma^{2}\left(\mathrm{~F}_{\mathrm{o}}{ }^{2}\right)+(0.02 \mathrm{P})^{2}\right]$ where $\mathrm{P}=\left(\mathrm{F}_{\mathrm{o}}{ }^{2}+2 \mathrm{~F}_{\mathrm{c}}{ }^{2}\right) / 3$

0.002

0.000

1.49 and $-1.48 \mathrm{e} \cdot \AA^{-3}$

\section{Programs Used}

Cell refinement

Bruker SMART v5.606, Bruker SAINT v6.29

Data collection

Bruker SMART v5.054

Data reduction

Bruker SAINT v6.29

Structure solution

SHELX-97 (Sheldrick, 1997)

Structure refinement

SHELX-97 (Sheldrick, 1997)

Graphics

Diamond, Bruker SHELXTL v6.12

\section{References}

Bruker (1999) SMART (v5.054), SMART (v5.606), SAINT (v6.29) and SHELXTL (v6.12). Bruker AXS Inc., Madison, Wisconsin, USA.

Diamond 2.1. (2000) Crystal Impact GbR, Bonn, Germany.

Spek, A.L. (1990). Acta Cryst., A46, C-34.

Sheldrick, G. M. (1997). SHELX-97. Program for Structures Refinement. Univ. of Gottingen, Federal Republic of Germany. 


\section{Special Refinement Details}

A colorless blade-shaped crystal was selected and mounted on a glass fiber with Paratone-N oil. Seven runs of data were collected with 30 second long, $-0.3^{\circ}$ wide $\omega$-scans at seven values of $\varphi(0,51,103,154,206,257$, and $309^{\circ}$ ) with the detector $5 \mathrm{~cm}$ (nominal) distant at a $\theta$ of $-28^{\circ}$. The initial cell for data reduction was calculated from 999 centered reflections chosen from throughout the data frames. A total of 2 reflections was discarded in the triclinic least-squares with a reciprocal lattice vector tolerance of 0.005 . For data processing with SAINT v6.29, all defaults were used, except: a fixed box size of $1.8 \times 1.8 \times 0.7$ was used, periodic orientation matrix updating was disabled, the instrument error was set to zero, no Laue class integration restraints were used, the I/ $\sigma$ lower limit and threshold were set to 5 and 4 respectively, and for the post-integration global least squares refinement, no constraints were applied. No decay correction was needed.

No reflections were specifically omitted from the final processed dataset; 1047 reflections were rejected, with 8 space group-absence violations, 7 inconsistent equivalents, and no reflections suppressed. Refinement of $\mathrm{F}^{2}$ was against all reflections. The weighted $\mathrm{R}$-factor $(w \mathrm{R})$ and goodness of fit $(\mathrm{S})$ are based on $\mathrm{F}^{2}$, conventional R-factors (R) are based on F, with $F$ set to zero for negative $F^{2}$. The threshold expression of $F^{2}>2 \sigma\left(F^{2}\right)$ is used only for calculating R-factors(gt) etc. and is not relevant to the choice of reflections for refinement.

The $\mathrm{N}$-methylpyrrolidene fragment is coordinated to the $\mathrm{Pd}$ metal by $\mathrm{C}(43)$ and $\mathrm{N}$. The difference map indicated this fragment was disordered. The structure was modeled as a superposition of two molecules in which $\mathrm{C}(43)$ and $\mathrm{N}$ interchange positions; the other ring carbon atoms in the two orientations overlap, and the $N$-methyl carbon occupies two different positions. (The minor position of the methyl carbon was refined isotropically.) The relative populations are 0.81 and 0.19 . The two models have different geometries; there is a difference of $\sim 15$ degrees in both the P-Pd-N and P-Pd-C angles. In at least one similar system, the ligand is disordered about the molecular 2fold axis bisecting the P-Pd-P angle, resulting in both models having the same geometry.

Of the twenty largest peaks in the final difference map, three are greater than $|1| \mathrm{e} \cdot \AA^{-3}$. All three peaks are near the palladium atom (within a distance of $0.88 \AA$ ), including the largest positive peak of $1.49 \mathrm{e} \cdot \AA^{-3}$ and the largest negative peak of $-1.48 \mathrm{e} \cdot \AA^{-3}$. 
Table 17. Atomic coordinates $\left(\times_{10}^{4}\right)$ and equivalent isotropic displacement parameters $\left(\AA^{2} \times 10^{3}\right)$ for 9 (CCDC 186800). U(eq) is defined as one third of the trace of the orthogonalized $\mathrm{U}^{\mathrm{ij}}$ tensor.

\begin{tabular}{|c|c|c|c|c|}
\hline & $\mathrm{x}$ & $\mathrm{y}$ & $\mathrm{z}$ & $\mathrm{U}_{\text {eq }}$ or $\mathrm{U}_{\text {iso }}$ \\
\hline $\mathrm{Pd}$ & $-343.9(2)$ & $1290.8(1)$ & $1749.2(2)$ & $24.2(1)$ \\
\hline $\mathrm{P}(1)$ & $-105.3(8)$ & $1626.2(3)$ & $3669.9(7)$ & $19.6(2)$ \\
\hline $\mathrm{P}(2)$ & $1606.2(7)$ & $957.5(3)$ & $2577.5(7)$ & $18.4(2)$ \\
\hline $\mathrm{N}^{\mathrm{a}}$ & $-1964(3)$ & $1408.3(15)$ & $96(3)$ & $63.4(12)$ \\
\hline $\mathrm{C}\left(43^{\prime}\right)^{\mathrm{b}}$ & $-1964(3)$ & $1408.3(15)$ & $96(3)$ & $63.4(12)$ \\
\hline B & $2455(3)$ & $1248.1(12)$ & $5266(3)$ & $19.5(7)$ \\
\hline $\mathrm{C}(1)$ & $948(3)$ & $1298.5(11)$ & $5007(3)$ & $20.0(7)$ \\
\hline $\mathrm{C}(2)$ & $2694(3)$ & $1254.2(10)$ & $3934(3)$ & $18.9(6)$ \\
\hline$C(3)$ & $3265(3)$ & $1665.7(10)$ & $6192(3)$ & $20.7(7)$ \\
\hline$C(4)$ & $3231(3)$ & $1715.7(11)$ & 7391(3) & $26.6(8)$ \\
\hline$C(5)$ & $3831(3)$ & 2068.7(12) & $8198(3)$ & $36.7(9)$ \\
\hline$C(6)$ & $4518(3)$ & 2386.3(12) & $7856(3)$ & $37.7(9)$ \\
\hline$C(7)$ & $4598(3)$ & $2345.8(11)$ & $6707(3)$ & $33.6(8)$ \\
\hline $\mathrm{C}(8)$ & $3969(3)$ & 1993.3(11) & $5886(3)$ & $26.2(7)$ \\
\hline C(9) & $3005(3)$ & 768.2(10) & $6040(3)$ & $18.3(7)$ \\
\hline$C(10)$ & $4305(3)$ & $695.9(11)$ & $6630(3)$ & $25.4(8)$ \\
\hline $\mathrm{C}(11)$ & $4825(3)$ & $302.9(12)$ & $7301(3)$ & 29.1(8) \\
\hline$C(12)$ & $4068(3)$ & $-37.7(11)$ & $7435(3)$ & $29.8(8)$ \\
\hline$C(13)$ & 2793(3) & $16.9(11)$ & $6857(3)$ & $28.2(8)$ \\
\hline$C(14)$ & 2281(3) & $408.7(10)$ & $6171(3)$ & $22.5(7)$ \\
\hline$C(15)$ & $-1543(3)$ & $1633.2(10)$ & $3969(3)$ & $20.3(7)$ \\
\hline$C(16)$ & $-1752(3)$ & $1946.9(12)$ & $4769(3)$ & $31.9(8)$ \\
\hline$C(17)$ & $-2772(3)$ & $1900.7(12)$ & $5104(3)$ & $36.7(9)$ \\
\hline $\mathrm{C}(18)$ & $-3604(3)$ & $1548.6(12)$ & $4622(3)$ & $31.3(8)$ \\
\hline C(19) & $-3418(3)$ & $1238.8(12)$ & $3818(3)$ & $31.9(8)$ \\
\hline $\mathrm{C}(20)$ & $-2402(3)$ & $1282.4(11)$ & $3499(3)$ & $27.6(7)$ \\
\hline $\mathrm{C}(21)$ & 397(3) & $2219.8(10)$ & $3907(3)$ & $22.4(7)$ \\
\hline $\mathrm{C}(22)$ & $1043(3)$ & 2409.1(11) & $5103(3)$ & $25.5(7)$ \\
\hline $\mathrm{C}(23)$ & $1402(3)$ & 2861.9(11) & $5232(3)$ & $31.7(8)$ \\
\hline$C(24)$ & $1107(4)$ & $3136.7(12)$ & $4182(4)$ & $41.2(10)$ \\
\hline$C(25)$ & $435(4)$ & $2958.4(13)$ & 2987(3) & $47.9(11)$ \\
\hline$C(26)$ & 99(4) & 2503.1(12) & $2852(3)$ & $38.2(9)$ \\
\hline $\mathrm{C}(27)$ & $1608(3)$ & 358.7(10) & $3007(3)$ & $18.0(7)$ \\
\hline $\mathrm{C}(28)$ & $2737(3)$ & $118.9(10)$ & $3568(3)$ & $20.9(7)$ \\
\hline C(29) & $2743(3)$ & $-335.5(11)$ & $3881(3)$ & $24.4(7)$ \\
\hline$C(30)$ & $1631(3)$ & $-562.2(11)$ & $3632(3)$ & $28.2(8)$ \\
\hline$C(31)$ & $508(3)$ & $-329.4(12)$ & $3065(3)$ & $31.0(8)$ \\
\hline $\mathrm{C}(32)$ & 501(3) & 129.9(11) & $2769(3)$ & $25.4(7)$ \\
\hline C(33) & $2379(3)$ & $920.1(10)$ & $1472(3)$ & $19.2(7)$ \\
\hline $\mathrm{C}(34)$ & $3369(3)$ & 1196.9(10) & $1559(3)$ & $23.5(7)$ \\
\hline$C(35)$ & $3912(3)$ & $1160.8(11)$ & $688(3)$ & $29.5(8)$ \\
\hline$C(36)$ & $3498(3)$ & 837.4(12) & $-256(3)$ & $29.8(8)$ \\
\hline $\mathrm{C}(37)$ & $2517(3)$ & $558.3(12)$ & $-349(3)$ & $32.3(9)$ \\
\hline $\mathrm{C}(38)$ & 1944(3) & $601.8(11)$ & 489(3) & $30.0(8)$ \\
\hline$C(39)^{a}$ & $-2124(5)$ & $1843.5(15)$ & $-569(5)$ & $52.0(17)$ \\
\hline $\mathrm{C}\left(39^{\prime}\right)^{\mathrm{b}}$ & $-1361(17)$ & $1377(6)$ & $-1196(17)$ & $32(6)^{\mathrm{c}}$ \\
\hline
\end{tabular}




\begin{tabular}{lrrrr}
$\mathrm{C}(40)$ & $-3072(5)$ & $1182.5(19)$ & $27(5)$ & $81.5(16)$ \\
$\mathrm{C}(41)$ & $-2759(5)$ & $691.8(19)$ & $238(4)$ & $79.7(18)$ \\
$\mathrm{C}(42)$ & $-1796(5)$ & $631.0(17)$ & $-348(4)$ & $67.0(14)$ \\
$\mathrm{C}(43)^{\mathrm{a}}$ & $1204(5)$ & $1091.8(16)$ & $-103(4)$ & $61.3(13)$ \\
$\mathrm{N}^{\mathrm{b}}$ & $1204(5)$ & $1091.8(16)$ & $-103(4)$ & $61.3(13)$ \\
\hline
\end{tabular}

Population $=0.815(7)$

${ }^{\mathrm{b}}$ Population $=0.185(7)$

${ }^{c} \mathrm{U}_{\text {iso }}$ 
Table 18. Bond lengths $[\AA ̊]$ and angles $\left[{ }^{\circ}\right]$ for 9 (CCDC 186800).

\begin{tabular}{|c|c|c|c|}
\hline Pd-C(43) & $2.048(4)$ & C(19)-C(20) & $1.375(4)$ \\
\hline Pd-N & $2.104(3)$ & $\mathrm{C}(19)-\mathrm{H}(19)$ & 0.9500 \\
\hline Pd-P(2) & $2.2945(8)$ & $\mathrm{C}(20)-\mathrm{H}(20)$ & 0.9500 \\
\hline Pd-P(1) & $2.3354(8)$ & $C(21)-C(22)$ & $1.394(4)$ \\
\hline $\mathrm{P}(1)-\mathrm{C}(1)$ & $1.812(3)$ & $C(21)-C(26)$ & $1.396(4)$ \\
\hline $\mathrm{P}(1)-\mathrm{C}(21)$ & $1.818(3)$ & $C(22)-C(23)$ & $1.380(4)$ \\
\hline$P(1)-C(15)$ & $1.835(3)$ & $\mathrm{C}(22)-\mathrm{H}(22)$ & 0.9500 \\
\hline $\mathrm{P}(2)-\mathrm{C}(2)$ & $1.793(3)$ & $C(23)-C(24)$ & $1.377(5)$ \\
\hline $\mathrm{P}(2)-\mathrm{C}(27)$ & $1.821(3)$ & $\mathrm{C}(23)-\mathrm{H}(23)$ & 0.9500 \\
\hline $\mathrm{P}(2)-\mathrm{C}(33)$ & $1.832(3)$ & $C(24)-C(25)$ & $1.386(5)$ \\
\hline N-C(43) & $1.361(5)$ & $\mathrm{C}(24)-\mathrm{H}(24)$ & 0.9500 \\
\hline N-C(40) & $1.420(6)$ & $C(25)-C(26)$ & $1.379(5)$ \\
\hline N-C(39) & $1.460(5)$ & $\mathrm{C}(25)-\mathrm{H}(25)$ & 0.9500 \\
\hline $\mathrm{B}-\mathrm{C}(3)$ & $1.648(4)$ & $\mathrm{C}(26)-\mathrm{H}(26)$ & 0.9500 \\
\hline $\mathrm{B}-\mathrm{C}(9)$ & $1.650(5)$ & $\mathrm{C}(27)-\mathrm{C}(32)$ & $1.376(4)$ \\
\hline $\mathrm{B}-\mathrm{C}(1)$ & $1.661(4)$ & $C(27)-C(28)$ & $1.398(4)$ \\
\hline $\mathrm{B}-\mathrm{C}(2)$ & $1.666(4)$ & $\mathrm{C}(28)-\mathrm{C}(29)$ & $1.377(4)$ \\
\hline $\mathrm{C}(1)-\mathrm{H}(1 \mathrm{~A})$ & 0.9900 & $\mathrm{C}(28)-\mathrm{H}(28)$ & 0.9500 \\
\hline $\mathrm{C}(1)-\mathrm{H}(1 \mathrm{~B})$ & 0.9900 & C(29)-C(30) & $1.376(4)$ \\
\hline $\mathrm{C}(2)-\mathrm{H}(2 \mathrm{~A})$ & 0.9900 & $\mathrm{C}(29)-\mathrm{H}(29)$ & 0.9500 \\
\hline $\mathrm{C}(2)-\mathrm{H}(2 \mathrm{~B})$ & 0.9900 & $C(30)-C(31)$ & $1.383(4)$ \\
\hline $\mathrm{C}(3)-\mathrm{C}(8)$ & $1.394(4)$ & $\mathrm{C}(30)-\mathrm{H}(30)$ & 0.9500 \\
\hline$C(3)-C(4)$ & $1.407(4)$ & $C(31)-C(32)$ & $1.386(4)$ \\
\hline$C(4)-C(5)$ & $1.378(4)$ & $\mathrm{C}(31)-\mathrm{H}(31)$ & 0.9500 \\
\hline $\mathrm{C}(4)-\mathrm{H}(4)$ & 0.9500 & $\mathrm{C}(32)-\mathrm{H}(32)$ & 0.9500 \\
\hline$C(5)-C(6)$ & $1.381(5)$ & C(33)-C(34) & $1.378(4)$ \\
\hline $\mathrm{C}(5)-\mathrm{H}(5)$ & 0.9500 & C(33)-C(38) & $1.396(4)$ \\
\hline$C(6)-C(7)$ & $1.370(5)$ & $C(34)-C(35)$ & $1.388(4)$ \\
\hline $\mathrm{C}(6)-\mathrm{H}(6)$ & 0.9500 & $\mathrm{C}(34)-\mathrm{H}(34)$ & 0.9500 \\
\hline$C(7)-C(8)$ & $1.394(4)$ & $C(35)-C(36)$ & $1.375(4)$ \\
\hline $\mathrm{C}(7)-\mathrm{H}(7)$ & 0.9500 & $\mathrm{C}(35)-\mathrm{H}(35)$ & 0.9500 \\
\hline C(8)-H(8) & 0.9500 & $C(36)-C(37)$ & $1.370(5)$ \\
\hline C(9)-C(14) & $1.393(4)$ & $\mathrm{C}(36)-\mathrm{H}(36)$ & 0.9500 \\
\hline C(9)-C(10) & $1.402(4)$ & $\mathrm{C}(37)-\mathrm{C}(38)$ & $1.382(4)$ \\
\hline$C(10)-C(11)$ & $1.383(4)$ & $\mathrm{C}(37)-\mathrm{H}(37)$ & 0.9500 \\
\hline $\mathrm{C}(10)-\mathrm{H}(10)$ & 0.9500 & $\mathrm{C}(38)-\mathrm{H}(38)$ & 0.9500 \\
\hline$C(11)-C(12)$ & $1.378(5)$ & $\mathrm{C}(39)-\mathrm{H}(39 \mathrm{~A})$ & 0.9800 \\
\hline $\mathrm{C}(11)-\mathrm{H}(11)$ & 0.9500 & C(39)-H(39B) & 0.9800 \\
\hline$C(12)-C(13)$ & $1.370(5)$ & C(39)-H(39C) & 0.9800 \\
\hline $\mathrm{C}(12)-\mathrm{H}(12)$ & 0.9500 & $\mathrm{C}\left(39^{\prime}\right)-\mathrm{H}(39 \mathrm{D})$ & 0.9800 \\
\hline$C(13)-C(14)$ & $1.385(4)$ & C(39')-H(39E) & 0.9800 \\
\hline $\mathrm{C}(13)-\mathrm{H}(13)$ & 0.9500 & $\mathrm{C}\left(39^{\prime}\right)-\mathrm{H}(39 \mathrm{~F})$ & 0.9800 \\
\hline $\mathrm{C}(14)-\mathrm{H}(14)$ & 0.9500 & $C(40)-C(41)$ & $1.478(6)$ \\
\hline$C(15)-C(20)$ & $1.383(4)$ & $\mathrm{C}(40)-\mathrm{H}(40 \mathrm{~A})$ & 0.9900 \\
\hline$C(15)-C(16)$ & $1.390(4)$ & $\mathrm{C}(40)-\mathrm{H}(40 \mathrm{~B})$ & 0.9900 \\
\hline$C(16)-C(17)$ & $1.392(5)$ & $C(41)-C(42)$ & $1.530(6)$ \\
\hline $\mathrm{C}(16)-\mathrm{H}(16)$ & 0.9500 & $\mathrm{C}(41)-\mathrm{H}(41 \mathrm{~A})$ & 0.9900 \\
\hline$C(17)-C(18)$ & $1.369(5)$ & $\mathrm{C}(41)-\mathrm{H}(41 \mathrm{~B})$ & 0.9900 \\
\hline $\mathrm{C}(17)-\mathrm{H}(17)$ & 0.9500 & $C(42)-C(43)$ & $1.488(6)$ \\
\hline C(18)-C(19) & $1.374(5)$ & $\mathrm{C}(42)-\mathrm{H}(42 \mathrm{~A})$ & 0.9900 \\
\hline $\mathrm{C}(18)-\mathrm{H}(18)$ & 0.9500 & $\mathrm{C}(42)-\mathrm{H}(42 \mathrm{~B})$ & 0.9900 \\
\hline
\end{tabular}




\begin{tabular}{|c|c|c|c|}
\hline $\mathrm{C}(43)-\mathrm{H}(43)$ & 1.0000 & $\mathrm{C}(5)-\mathrm{C}(6)-\mathrm{H}(6)$ & 120.4 \\
\hline C(43)-Pd-N & $38.25(16)$ & $\mathrm{C}(6)-\mathrm{C}(7)-\mathrm{C}(8)$ & 120.1(3) \\
\hline C(43)-Pd-P(2) & $107.58(15)$ & $\mathrm{C}(6)-\mathrm{C}(7)-\mathrm{H}(7)$ & 120.0 \\
\hline N-Pd-P(2) & $145.42(13)$ & $\mathrm{C}(8)-\mathrm{C}(7)-\mathrm{H}(7)$ & 120.0 \\
\hline C(43)-Pd-P(1) & $158.75(15)$ & $C(3)-C(8)-C(7)$ & $122.6(3)$ \\
\hline N-Pd-P(1) & $120.96(13)$ & $\mathrm{C}(3)-\mathrm{C}(8)-\mathrm{H}(8)$ & 118.7 \\
\hline $\mathrm{P}(2)-\mathrm{Pd}-\mathrm{P}(1)$ & $93.49(3)$ & $\mathrm{C}(7)-\mathrm{C}(8)-\mathrm{H}(8)$ & 118.7 \\
\hline $\mathrm{C}(1)-\mathrm{P}(1)-\mathrm{C}(21)$ & 108.11(14) & $C(14)-C(9)-C(10)$ & $114.4(3)$ \\
\hline C(1)-P(1)-C(15) & 101.61(13) & C(14)-C(9)-B & $125.6(3)$ \\
\hline $\mathrm{C}(21)-\mathrm{P}(1)-\mathrm{C}(15)$ & $102.87(14)$ & C(10)-C(9)-B & $120.0(3)$ \\
\hline $\mathrm{C}(1)-\mathrm{P}(1)-\mathrm{Pd}$ & $111.59(10)$ & $\mathrm{C}(11)-\mathrm{C}(10)-\mathrm{C}(9)$ & $122.8(3)$ \\
\hline $\mathrm{C}(21)-\mathrm{P}(1)-\mathrm{Pd}$ & $116.95(10)$ & $\mathrm{C}(11)-\mathrm{C}(10)-\mathrm{H}(10)$ & 118.6 \\
\hline C(15)-P(1)-Pd & $114.28(10)$ & $\mathrm{C}(9)-\mathrm{C}(10)-\mathrm{H}(10)$ & 118.6 \\
\hline $\mathrm{C}(2)-\mathrm{P}(2)-\mathrm{C}(27)$ & $107.76(14)$ & $C(12)-C(11)-C(10)$ & $120.6(3)$ \\
\hline C(2)-P(2)-C(33) & $105.39(14)$ & $\mathrm{C}(12)-\mathrm{C}(11)-\mathrm{H}(11)$ & 119.7 \\
\hline $\mathrm{C}(27)-\mathrm{P}(2)-\mathrm{C}(33)$ & 100.61(13) & $\mathrm{C}(10)-\mathrm{C}(11)-\mathrm{H}(11)$ & 119.7 \\
\hline $\mathrm{C}(2)-\mathrm{P}(2)-\mathrm{Pd}$ & $113.02(10)$ & $C(13)-C(12)-C(11)$ & $118.4(3)$ \\
\hline$C(27)-P(2)-P d$ & $114.57(10)$ & $\mathrm{C}(13)-\mathrm{C}(12)-\mathrm{H}(12)$ & 120.8 \\
\hline $\mathrm{C}(33)-\mathrm{P}(2)-\mathrm{Pd}$ & $114.37(10)$ & $\mathrm{C}(11)-\mathrm{C}(12)-\mathrm{H}(12)$ & 120.8 \\
\hline $\mathrm{C}(43)-\mathrm{N}-\mathrm{C}(40)$ & $108.0(4)$ & $C(12)-C(13)-C(14)$ & $120.6(3)$ \\
\hline C(43)-N-C(39) & $117.4(4)$ & $\mathrm{C}(12)-\mathrm{C}(13)-\mathrm{H}(13)$ & 119.7 \\
\hline C(40)-N-C(39) & $117.0(4)$ & $\mathrm{C}(14)-\mathrm{C}(13)-\mathrm{H}(13)$ & 119.7 \\
\hline $\mathrm{C}(43)-\mathrm{N}-\mathrm{Pd}$ & $68.6(2)$ & $\mathrm{C}(13)-\mathrm{C}(14)-\mathrm{C}(9)$ & $123.2(3)$ \\
\hline C(40)-N-Pd & $115.9(3)$ & $\mathrm{C}(13)-\mathrm{C}(14)-\mathrm{H}(14)$ & 118.4 \\
\hline C(39)-N-Pd & $119.9(3)$ & C(9)-C(14)-H(14) & 118.4 \\
\hline C(3)-B-C(9) & $106.5(2)$ & $C(20)-C(15)-C(16)$ & $117.4(3)$ \\
\hline$C(3)-B-C(1)$ & $109.8(2)$ & $\mathrm{C}(20)-\mathrm{C}(15)-\mathrm{P}(1)$ & 119.1(2) \\
\hline C(9)-B-C(1) & $109.0(2)$ & $\mathrm{C}(16)-\mathrm{C}(15)-\mathrm{P}(1)$ & $123.1(2)$ \\
\hline C(3)-B-C(2) & 109.1(2) & $C(15)-C(16)-C(17)$ & $120.9(3)$ \\
\hline $\mathrm{C}(9)-\mathrm{B}-\mathrm{C}(2)$ & $109.7(2)$ & $\mathrm{C}(15)-\mathrm{C}(16)-\mathrm{H}(16)$ & 119.5 \\
\hline $\mathrm{C}(1)-\mathrm{B}-\mathrm{C}(2)$ & $112.7(2)$ & $\mathrm{C}(17)-\mathrm{C}(16)-\mathrm{H}(16)$ & 119.5 \\
\hline $\mathrm{B}-\mathrm{C}(1)-\mathrm{P}(1)$ & $120.3(2)$ & $C(18)-C(17)-C(16)$ & $120.1(3)$ \\
\hline $\mathrm{B}-\mathrm{C}(1)-\mathrm{H}(1 \mathrm{~A})$ & 107.3 & $\mathrm{C}(18)-\mathrm{C}(17)-\mathrm{H}(17)$ & 120.0 \\
\hline $\mathrm{P}(1)-\mathrm{C}(1)-\mathrm{H}(1 \mathrm{~A})$ & 107.3 & $\mathrm{C}(16)-\mathrm{C}(17)-\mathrm{H}(17)$ & 120.0 \\
\hline $\mathrm{B}-\mathrm{C}(1)-\mathrm{H}(1 \mathrm{~B})$ & 107.3 & $C(17)-C(18)-C(19)$ & $119.6(3)$ \\
\hline $\mathrm{P}(1)-\mathrm{C}(1)-\mathrm{H}(1 \mathrm{~B})$ & 107.3 & $\mathrm{C}(17)-\mathrm{C}(18)-\mathrm{H}(18)$ & 120.2 \\
\hline $\mathrm{H}(1 \mathrm{~A})-\mathrm{C}(1)-\mathrm{H}(1 \mathrm{~B})$ & 106.9 & $\mathrm{C}(19)-\mathrm{C}(18)-\mathrm{H}(18)$ & 120.2 \\
\hline $\mathrm{B}-\mathrm{C}(2)-\mathrm{P}(2)$ & $118.7(2)$ & $C(18)-C(19)-C(20)$ & $120.2(3)$ \\
\hline $\mathrm{B}-\mathrm{C}(2)-\mathrm{H}(2 \mathrm{~A})$ & 107.6 & $\mathrm{C}(18)-\mathrm{C}(19)-\mathrm{H}(19)$ & 119.9 \\
\hline $\mathrm{P}(2)-\mathrm{C}(2)-\mathrm{H}(2 \mathrm{~A})$ & 107.6 & $\mathrm{C}(20)-\mathrm{C}(19)-\mathrm{H}(19)$ & 119.9 \\
\hline $\mathrm{B}-\mathrm{C}(2)-\mathrm{H}(2 \mathrm{~B})$ & 107.6 & $C(19)-C(20)-C(15)$ & $121.8(3)$ \\
\hline $\mathrm{P}(2)-\mathrm{C}(2)-\mathrm{H}(2 \mathrm{~B})$ & 107.6 & $\mathrm{C}(19)-\mathrm{C}(20)-\mathrm{H}(20)$ & 119.1 \\
\hline $\mathrm{H}(2 \mathrm{~A})-\mathrm{C}(2)-\mathrm{H}(2 \mathrm{~B})$ & 107.1 & $\mathrm{C}(15)-\mathrm{C}(20)-\mathrm{H}(20)$ & 119.1 \\
\hline$C(8)-C(3)-C(4)$ & $115.3(3)$ & $C(22)-C(21)-C(26)$ & $118.3(3)$ \\
\hline C(8)-C(3)-B & $125.9(3)$ & C(22)-C(21)-P(1) & $122.8(2)$ \\
\hline$C(4)-C(3)-B$ & $118.7(3)$ & $\mathrm{C}(26)-\mathrm{C}(21)-\mathrm{P}(1)$ & $118.9(2)$ \\
\hline $\mathrm{C}(5)-\mathrm{C}(4)-\mathrm{C}(3)$ & $122.4(3)$ & $C(23)-C(22)-C(21)$ & $120.5(3)$ \\
\hline $\mathrm{C}(5)-\mathrm{C}(4)-\mathrm{H}(4)$ & 118.8 & $\mathrm{C}(23)-\mathrm{C}(22)-\mathrm{H}(22)$ & 119.7 \\
\hline $\mathrm{C}(3)-\mathrm{C}(4)-\mathrm{H}(4)$ & 118.8 & $\mathrm{C}(21)-\mathrm{C}(22)-\mathrm{H}(22)$ & 119.7 \\
\hline$C(4)-C(5)-C(6)$ & $120.4(3)$ & $C(24)-C(23)-C(22)$ & $120.5(3)$ \\
\hline $\mathrm{C}(4)-\mathrm{C}(5)-\mathrm{H}(5)$ & 119.8 & $\mathrm{C}(24)-\mathrm{C}(23)-\mathrm{H}(23)$ & 119.7 \\
\hline $\mathrm{C}(6)-\mathrm{C}(5)-\mathrm{H}(5)$ & 119.8 & $\mathrm{C}(22)-\mathrm{C}(23)-\mathrm{H}(23)$ & 119.7 \\
\hline$C(7)-C(6)-C(5)$ & $119.2(3)$ & $\mathrm{C}(23)-\mathrm{C}(24)-\mathrm{C}(25)$ & $119.8(3)$ \\
\hline $\mathrm{C}(7)-\mathrm{C}(6)-\mathrm{H}(6)$ & 120.4 & $\mathrm{C}(23)-\mathrm{C}(24)-\mathrm{H}(24)$ & 120.1 \\
\hline
\end{tabular}




$\begin{array}{ll}\mathrm{C}(25)-\mathrm{C}(24)-\mathrm{H}(24) & 120.1 \\ \mathrm{C}(26)-\mathrm{C}(25)-\mathrm{C}(24) & 119.8(3) \\ \mathrm{C}(26)-\mathrm{C}(25)-\mathrm{H}(25) & 120.1 \\ \mathrm{C}(24)-\mathrm{C}(25)-\mathrm{H}(25) & 120.1 \\ \mathrm{C}(25)-\mathrm{C}(26)-\mathrm{C}(21) & 121.0(3) \\ \mathrm{C}(25)-\mathrm{C}(26)-\mathrm{H}(26) & 119.5 \\ \mathrm{C}(21)-\mathrm{C}(26)-\mathrm{H}(26) & 119.5 \\ \mathrm{C}(32)-\mathrm{C}(27)-\mathrm{C}(28) & 118.5(3) \\ \mathrm{C}(32)-\mathrm{C}(27)-\mathrm{P}(2) & 121.0(2) \\ \mathrm{C}(28)-\mathrm{C}(27)-\mathrm{P}(2) & 120.5(2) \\ \mathrm{C}(29)-\mathrm{C}(28)-\mathrm{C}(27) & 120.7(3) \\ \mathrm{C}(29)-\mathrm{C}(28)-\mathrm{H}(28) & 119.6 \\ \mathrm{C}(27)-\mathrm{C}(28)-\mathrm{H}(28) & 119.6 \\ \mathrm{C}(30)-\mathrm{C}(29)-\mathrm{C}(28) & 120.4(3) \\ \mathrm{C}(30)-\mathrm{C}(29)-\mathrm{H}(29) & 119.8 \\ \mathrm{C}(28)-\mathrm{C}(29)-\mathrm{H}(29) & 119.8 \\ \mathrm{C}(29)-\mathrm{C}(30)-\mathrm{C}(31) & 119.4(3) \\ \mathrm{C}(29)-\mathrm{C}(30)-\mathrm{H}(30) & 120.3 \\ \mathrm{C}(31)-\mathrm{C}(30)-\mathrm{H}(30) & 120.3 \\ \mathrm{C}(30)-\mathrm{C}(31)-\mathrm{C}(32) & 120.3(3) \\ \mathrm{C}(30)-\mathrm{C}(31)-\mathrm{H}(31) & 119.8 \\ \mathrm{C}(32)-\mathrm{C}(31)-\mathrm{H}(31) & 119.8 \\ \mathrm{C}(27)-\mathrm{C}(32)-\mathrm{C}(31) & 120.7(3) \\ \mathrm{C}(27)-\mathrm{C}(32)-\mathrm{H}(32) & 119.7 \\ \mathrm{C}(31)-\mathrm{C}(32)-\mathrm{H}(32) & 119.7 \\ \mathrm{C}(34)-\mathrm{C}(33)-\mathrm{C}(38) & 118.2(3) \\ \mathrm{C}(34)-\mathrm{C}(33)-\mathrm{P}(2) & 122.8(2) \\ \mathrm{C}(38)-\mathrm{C}(33)-\mathrm{P}(2) & 119.1(2) \\ \mathrm{C}(33)-\mathrm{C}(34)-\mathrm{C}(35) & 120.7(3) \\ \mathrm{C}(33)-\mathrm{C}(34)-\mathrm{H}(34) & 119.6 \\ \mathrm{C}(35)-\mathrm{C}(34)-\mathrm{H}(34) & 119.6 \\ \mathrm{C}(36)-\mathrm{C}(35)-\mathrm{C}(34) & 120.5(3) \\ \mathrm{C}(36)-\mathrm{C}(35)-\mathrm{H}(35) & 119.7 \\ \mathrm{C}(34)-\mathrm{C}(35)-\mathrm{H}(35) & 119.7 \\ \mathrm{C}(37)-\mathrm{C}(36)-\mathrm{C}(35) & 119.3(3) \\ & \end{array}$

$\begin{array}{ll}\text { C(37)-C(36)-H(36) } & 120.3 \\ \text { C(35)-C(36)-H(36) } & 120.3 \\ \text { C(36)-C(37)-C(38) } & 120.5(3) \\ \text { C(36)-C(37)-H(37) } & 119.7 \\ \text { C(38)-C(37)-H(37) } & 119.7 \\ \text { C(37)-C(38)-C(33) } & 120.7(3) \\ \text { C(37)-C(38)-H(38) } & 119.7 \\ \text { C(33)-C(38)-H(38) } & 119.7 \\ \mathrm{H}(39 D)-C(39 ')-H(39 E) & 109.5 \\ \text { H(39D)-C(39')-H(39F) } & 109.5 \\ \text { H(39E)-C(39')-H(39F) } & 109.5 \\ \text { N-C(40)-C(41) } & 106.5(4) \\ \text { N-C(40)-H(40A) } & 110.4 \\ \text { C(41)-C(40)-H(40A) } & 110.4 \\ \text { N-C(40)-H(40B) } & 110.4 \\ \text { C(41)-C(40)-H(40B) } & 110.4 \\ \text { H(40A)-C(40)-H(40B) } & 108.6 \\ \text { C(40)-C(41)-C(42) } & 102.5(4) \\ \text { C(40)-C(41)-H(41A) } & 111.3 \\ \text { C(42)-C(41)-H(41A) } & 111.3 \\ \text { C(40)-C(41)-H(41B) } & 111.3 \\ \text { C(42)-C(41)-H(41B) } & 111.3 \\ \text { H(41A)-C(41)-H(41B) } & 109.2 \\ \text { C(43)-C(42)-C(41) } & 100.0(4) \\ \text { C(43)-C(42)-H(42A) } & 111.8 \\ \text { C(41)-C(42)-H(42A) } & 111.8 \\ \text { C(43)-C(42)-H(42B) } & 111.8 \\ \text { C(41)-C(42)-H(42B) } & 111.8 \\ \text { H(42A)-C(42)-H(42B) } & 109.5 \\ \text { N-C(43)-C(42) } & 111.5(4) \\ \text { N-C(43)-Pd } & 73.1(2) \\ \text { C(42)-C(43)-Pd } & 116.8(3) \\ \text { N-C(43)-H(43) } & 116.1 \\ \text { C(42)-C(43)-H(43) } & 116.1 \\ \text { Pd-C(43)-H(43) } & 116.1 \\ & \\ & \end{array}$


Table 19. Anisotropic displacement parameters $\left(\AA^{2} \times 10^{4}\right)$ for 9 (CCDC 186800). The anisotropic displacement factorexponent takes the form: $-2 \pi^{2}\left[h^{2} a *{ }^{2} U^{11}+\ldots+\right.$ 2 h k a* b* $\mathbf{U}^{12}$ ]

\begin{tabular}{|c|c|c|c|c|c|c|}
\hline & $\mathrm{U}^{11}$ & $\mathrm{U}^{22}$ & $\mathrm{U}^{33}$ & $\mathrm{U}^{23}$ & $\mathrm{U}^{13}$ & $\mathrm{U}^{12}$ \\
\hline$\overline{P d}$ & $266(1)$ & $269(1)$ & $153(1)$ & $-45(1)$ & $44(1)$ & $47(1)$ \\
\hline $\mathrm{P}(1)$ & $236(5)$ & $174(4)$ & $163(4)$ & $-26(3)$ & $64(3)$ & $18(3)$ \\
\hline $\mathrm{P}(2)$ & 221(4) & $190(4)$ & 151(4) & $-17(3)$ & $85(3)$ & $6(3)$ \\
\hline $\mathrm{N}$ & $320(20)$ & $850(30)$ & $490(20)$ & $170(20)$ & $-92(18)$ & $-80(20)$ \\
\hline$C\left(43^{\prime}\right)$ & $320(20)$ & $850(30)$ & $490(20)$ & $170(20)$ & $-92(18)$ & $-80(20)$ \\
\hline B & $218(18)$ & $200(19)$ & 161(16) & $-20(15)$ & $69(14)$ & $-6(16)$ \\
\hline$C(1)$ & $240(16)$ & $198(16)$ & $181(15)$ & 6(14) & $103(13)$ & $55(15)$ \\
\hline$C(2)$ & $222(16)$ & $155(16)$ & 193(15) & $-1(13)$ & $87(13)$ & $7(14)$ \\
\hline$C(3)$ & $218(17)$ & 189(17) & 193(16) & $10(13)$ & $61(13)$ & $19(14)$ \\
\hline$C(4)$ & $380(20)$ & 201(18) & $177(16)$ & $18(13)$ & $67(15)$ & $12(15)$ \\
\hline$C(5)$ & $520(20)$ & $320(20)$ & 181(17) & $-59(15)$ & $56(17)$ & $31(18)$ \\
\hline$C(6)$ & $430(20)$ & $250(20)$ & $320(20)$ & $-101(16)$ & $14(18)$ & $-64(17)$ \\
\hline$C(7)$ & $287(19)$ & $269(19)$ & $400(20)$ & $6(17)$ & $77(16)$ & $-54(17)$ \\
\hline $\mathrm{C}(8)$ & $250(18)$ & $274(19)$ & $245(17)$ & $-36(15)$ & $82(15)$ & $-6(15)$ \\
\hline $\mathrm{C}(9)$ & $227(17)$ & $218(17)$ & $125(15)$ & $-62(12)$ & $91(13)$ & $4(14)$ \\
\hline$C(10)$ & $243(19)$ & $284(19)$ & $231(17)$ & $-39(14)$ & $92(15)$ & $31(14)$ \\
\hline $\mathrm{C}(11)$ & $275(19)$ & $350(20)$ & $220(17)$ & $-48(15)$ & $72(15)$ & $105(16)$ \\
\hline$C(12)$ & $490(20)$ & $250(19)$ & 174(17) & $32(14)$ & $158(16)$ & $149(17)$ \\
\hline$C(13)$ & $430(20)$ & $237(19)$ & $248(18)$ & $14(14)$ & 214(17) & $27(16)$ \\
\hline$C(14)$ & $265(18)$ & $250(18)$ & 191(16) & $-20(13)$ & $126(14)$ & $36(14)$ \\
\hline$C(15)$ & $214(17)$ & $180(17)$ & 199(16) & 2(13) & $65(13)$ & $20(14)$ \\
\hline$C(16)$ & $330(20)$ & $260(20)$ & $360(20)$ & $-90(16)$ & $133(17)$ & $-14(16)$ \\
\hline$C(17)$ & $350(20)$ & $360(20)$ & $450(20)$ & $-94(18)$ & $221(19)$ & $37(18)$ \\
\hline $\mathrm{C}(18)$ & $214(19)$ & $350(20)$ & $370(20)$ & $60(17)$ & $119(16)$ & $62(16)$ \\
\hline$C(19)$ & $254(18)$ & $290(20)$ & $348(19)$ & $3(16)$ & $48(15)$ & $-42(16)$ \\
\hline $\mathrm{C}(20)$ & $300(18)$ & $228(17)$ & 271(17) & $-65(16)$ & $81(14)$ & $4(17)$ \\
\hline $\mathrm{C}(21)$ & $258(18)$ & 204(17) & $226(17)$ & $13(13)$ & $114(14)$ & $0(14)$ \\
\hline$C(22)$ & $265(19)$ & $236(18)$ & $245(18)$ & $-6(14)$ & $81(15)$ & $30(15)$ \\
\hline$C(23)$ & $360(20)$ & $270(20)$ & 288(19) & $-52(15)$ & $94(16)$ & $-24(16)$ \\
\hline$C(24)$ & $540(30)$ & $290(20)$ & $410(20)$ & $-44(17)$ & $190(20)$ & $-172(18)$ \\
\hline$C(25)$ & $800(30)$ & $350(20)$ & $290(20)$ & $59(17)$ & $230(20)$ & $-140(20)$ \\
\hline$C(26)$ & $620(30)$ & $310(20)$ & 211(18) & $-39(15)$ & $156(18)$ & $-130(19)$ \\
\hline$C(27)$ & $239(17)$ & $179(16)$ & $153(15)$ & $-29(12)$ & $109(13)$ & $-14(13)$ \\
\hline $\mathrm{C}(28)$ & $235(18)$ & $230(18)$ & $188(16)$ & $-43(13)$ & $112(14)$ & $-22(14)$ \\
\hline $\mathrm{C}(29)$ & $275(19)$ & $246(19)$ & $216(17)$ & $-5(14)$ & $102(15)$ & $63(15)$ \\
\hline $\mathrm{C}(30)$ & $390(20)$ & $209(18)$ & 288(19) & $25(14)$ & $172(17)$ & $-8(16)$ \\
\hline $\mathrm{C}(31)$ & $280(20)$ & $300(20)$ & $380(20)$ & $21(16)$ & $158(17)$ & $-51(16)$ \\
\hline $\mathrm{C}(32)$ & $234(18)$ & $277(19)$ & 264(18) & $54(14)$ & $112(15)$ & $43(15)$ \\
\hline $\mathrm{C}(33)$ & $241(17)$ & $190(17)$ & $178(15)$ & $23(13)$ & $119(13)$ & $56(14)$ \\
\hline $\mathrm{C}(34)$ & 261(18) & $260(20)$ & $175(15)$ & $2(13)$ & $78(14)$ & $12(14)$ \\
\hline $\mathrm{C}(35)$ & $276(19)$ & $360(20)$ & 274(18) & $77(15)$ & $143(16)$ & $27(15)$ \\
\hline$C(36)$ & $370(20)$ & $370(20)$ & $219(17)$ & $91(15)$ & $183(16)$ & $137(17)$ \\
\hline $\mathrm{C}(37)$ & $470(20)$ & $330(20)$ & $207(18)$ & $-67(15)$ & $175(17)$ & 7(18) \\
\hline $\mathrm{C}(38)$ & $360(20)$ & $300(20)$ & $279(19)$ & $-60(15)$ & $172(17)$ & $-38(16)$ \\
\hline C(39) & $520(30)$ & $290(30)$ & $450(30)$ & $140(20)$ & $-120(20)$ & $140(20)$ \\
\hline
\end{tabular}




\begin{tabular}{lrrrrrr}
$\mathrm{C}(40)$ & $770(40)$ & $900(40)$ & $660(40)$ & $230(30)$ & $170(30)$ & $-40(30)$ \\
$\mathrm{C}(41)$ & $780(40)$ & $1080(50)$ & $390(30)$ & $0(30)$ & $90(30)$ & $-620(30)$ \\
$\mathrm{C}(42)$ & $890(40)$ & $790(40)$ & $390(30)$ & $-200(20)$ & $310(30)$ & $-380(30)$ \\
$\mathrm{C}(43)$ & $780(30)$ & $650(30)$ & $300(20)$ & $-160(20)$ & $120(20)$ & $-120(30)$ \\
$\mathrm{N}^{\prime}$ & $780(30)$ & $650(30)$ & $300(20)$ & $-160(20)$ & $120(20)$ & $-120(30)$ \\
\hline
\end{tabular}


Table 20. Hydrogen coordinates $\left(x 10^{3}\right)$ and isotropic displacement parameters $\left(\AA^{2} \times 10^{3}\right)$ for 9 (CCDC 186800).

\begin{tabular}{|c|c|c|c|c|}
\hline & $\mathrm{x}$ & $\mathrm{y}$ & z & $\mathrm{U}_{\text {iso }}$ \\
\hline $\mathrm{H}\left(43^{\prime}\right)^{\mathrm{b}}$ & -206 & 171 & -33 & 76 \\
\hline $\mathrm{H}(1 \mathrm{~A})$ & 91 & 143 & 578 & 24 \\
\hline $\mathrm{H}(1 \mathrm{~B})$ & 60 & 99 & 492 & 24 \\
\hline $\mathrm{H}(2 \mathrm{~A})$ & 354 & 113 & 413 & 23 \\
\hline $\mathrm{H}(2 \mathrm{~B})$ & 271 & 158 & 369 & 23 \\
\hline $\mathrm{H}(4)$ & 278 & 150 & 766 & 32 \\
\hline $\mathrm{H}(5)$ & 377 & 209 & 899 & 44 \\
\hline $\mathrm{H}(6)$ & 493 & 263 & 841 & 45 \\
\hline $\mathrm{H}(7)$ & 508 & 256 & 647 & 40 \\
\hline $\mathrm{H}(8)$ & 402 & 198 & 508 & 31 \\
\hline $\mathrm{H}(10)$ & 485 & 93 & 657 & 30 \\
\hline $\mathrm{H}(11)$ & 571 & 27 & 767 & 35 \\
\hline $\mathrm{H}(12)$ & 442 & -30 & 792 & 36 \\
\hline $\mathrm{H}(13)$ & 225 & -22 & 693 & 34 \\
\hline $\mathrm{H}(14)$ & 139 & 43 & 577 & 27 \\
\hline $\mathrm{H}(16)$ & -119 & 220 & 509 & 38 \\
\hline $\mathrm{H}(17)$ & -289 & 211 & 567 & 44 \\
\hline $\mathrm{H}(18)$ & -431 & 152 & 484 & 38 \\
\hline $\mathrm{H}(19)$ & -399 & 99 & 348 & 38 \\
\hline $\mathrm{H}(20)$ & -229 & 107 & 294 & 33 \\
\hline $\mathrm{H}(22)$ & 124 & 222 & 584 & 31 \\
\hline $\mathrm{H}(23)$ & 186 & 299 & 605 & 38 \\
\hline $\mathrm{H}(24)$ & 136 & 345 & 428 & 49 \\
\hline $\mathrm{H}(25)$ & 21 & 315 & 226 & 57 \\
\hline $\mathrm{H}(26)$ & -34 & 238 & 203 & 46 \\
\hline $\mathrm{H}(28)$ & 351 & 27 & 374 & 25 \\
\hline $\mathrm{H}(29)$ & 352 & -49 & 427 & 29 \\
\hline $\mathrm{H}(30)$ & 164 & -88 & 385 & 34 \\
\hline $\mathrm{H}(31)$ & -26 & -49 & 288 & 37 \\
\hline $\mathrm{H}(32)$ & -28 & 29 & 240 & 31 \\
\hline $\mathrm{H}(34)$ & 368 & 142 & 222 & 28 \\
\hline $\mathrm{H}(35)$ & 458 & 136 & 74 & 35 \\
\hline $\mathrm{H}(36)$ & 389 & 81 & -84 & 36 \\
\hline $\mathrm{H}(37)$ & 223 & 33 & -100 & 39 \\
\hline $\mathrm{H}(38)$ & 125 & 41 & 40 & 36 \\
\hline $\mathrm{H}(39 \mathrm{~A})^{\mathrm{a}}$ & -130 & 197 & -41 & 62 \\
\hline$H(39 B)^{a}$ & -259 & 206 & -26 & 62 \\
\hline$H(39 C)^{a}$ & -259 & 179 & -148 & 62 \\
\hline $\mathrm{H}(39 \mathrm{D})^{\mathrm{b}}$ & -152 & 169 & -102 & 38 \\
\hline $\mathrm{H}(39 \mathrm{E})^{\mathrm{b}}$ & -207 & 126 & -194 & 38 \\
\hline $\mathrm{H}(39 \mathrm{~F})^{\mathrm{b}}$ & -60 & 136 & -136 & 38 \\
\hline $\mathrm{H}(40 \mathrm{~A})$ & -376 & 123 & -81 & 98 \\
\hline $\mathrm{H}(40 \mathrm{~B})$ & -334 & 130 & 68 & 98 \\
\hline $\mathrm{H}(41 \mathrm{~A})$ & -351 & 50 & -19 & 96 \\
\hline $\mathrm{H}(41 \mathrm{~B})$ & -239 & 62 & 115 & 96 \\
\hline $\mathrm{H}(42 \mathrm{~A})$ & -118 & 39 & 8 & 80 \\
\hline
\end{tabular}


H(42B)

$-126$

$\mathrm{H}(43)^{\mathrm{a}} \quad-78$

${ }^{a}$ Population $=0.815(7)$

${ }^{\mathrm{b}}$ Population $=0.185(7)$ 